\title{
Design of Brønsted Acid-assisted Chiral Brønsted Acid (Chiral BBA) Catalyst Bearing a Bis(triflyl)methyl Group for a Mannich-type Reaction
}

\author{
Aiko Hasegawa, ${ }^{\dagger}$ Yuki Naganawa, ${ }^{\dagger}$ Makoto Fushimi, ${ }^{\dagger}$ Kazuaki Ishihara, ${ }^{*}{ }^{\dagger}$ \\ and Hisashi Yamamoto, ** \\ ${ }^{\dagger}$ Graduate School of Engineering, Nagoya University, Furo-cho, Chikusa, Nagoya 464-8603, Japan \\ ${ }^{*}$ Department of Chemistry, The University of Chicago, 5735 S. Ellis Avenue, Chicago, Illinois \\ 60637 \\ ishihara@cc.nagoya-u.ac.jp; yamamoto@uchicago.edu
}

General Methods. Infrared (IR) spectra were recorded on a JASCO FT/IR 460 plus spectrometer. $\quad{ }^{1} \mathrm{H}$ NMR spectra were measured on Varian Gemini-2000 (300 MHz) and VXR 500 (500 MHz) spectrometers at ambient temperature. Data were recorded as follows: chemical shift in $\mathrm{ppm}$ from internal tetramethylsilane on the $\delta$ scale, multiplicity $(\mathrm{s}=\operatorname{singlet} ; \mathrm{d}=$ doublet; $\mathrm{t}=$ triplet; $\mathrm{q}=$ quartet, $\mathrm{m}=$ multiplet $)$, coupling constant $(\mathrm{Hz})$, integration, and assignment. ${ }^{13} \mathrm{C} \mathrm{NMR}$ spectra were measured on Varian Gemini-2000 (75 MHz) and VXR 500 (125 MHz) spectrometers. Chemical shifts were recorded in ppm from the solvent resonance employed as the internal standard (deuterochloroform at $77.00 \mathrm{ppm}$ ). $\quad{ }^{19} \mathrm{~F}$ NMR spectra were measured on Varian Gemini-2000 (282 $\mathrm{MHz}$ ). Chemical shifts were recorded in ppm from the solvent resonance employed as the internal standard $(\alpha, \alpha, \alpha$-trifluorotoluene at $-64.00 \mathrm{ppm})$. High performance liquid chromatography (HPLC) analysis was conducted using Shimadzu LC-10 AD coupled diode array-detector SPD-MA-10A-VP and chiral column of Daicel CHIRALCEL OD-H $(4.6 \mathrm{~mm} \times 25 \mathrm{~cm})$, Daicel CHIRALCEL OJ-H $(4.6 \mathrm{~mm} \times 25 \mathrm{~cm})$, Daicel CHIRALPAK AD-H $(4.6 \mathrm{~mm} \times 25 \mathrm{~cm})$ or Daicel CHIRALPAK AS-H $(4.6 \mathrm{~mm} \times 25 \mathrm{~cm})$. Optical rotations were measured on a RUDOLPH AUTOPOL IV digital polarimeter. Melting points were determined using a Yanaco MP-J3. For thin-layer chromatography (TLC) analysis throughout this work, Merck precoated TLC plate (silica gel 60 GF254 $0.25 \mathrm{~mm}$ was used. The products were purified by column chromatography on silica gel (E. Merck Art. 9385 or Fuji Silysia Chemical Ltd. Cromatorex ${ }^{\circledR}$ NH-DM1020). Microanalyses and high resolution mass spectral analysis (HRMS) were performed at Chemical Instrument Center, Nagoya University. In experiments that required dry solvent, ether, $N, N$-dimethylformamide (DMF) and tetrahydorofuran (THF) were purchased from TCI or Wako as the "anhydrous" and stored over 4A molecular sieves. Benzene, hexane, toluene, and dichloromethane were freshly distilled from calcium hydride. Other simple chemicals were 
analytical-grade and obtained commercially.

\section{Preparation of $(R)$-2-Bis(trifluoromethanesulfonyl)methyl-2'-hydroxy-1,1'-binaphthyl} ((R)- 1)<smiles>COc1ccc2ccccc2c1-c1c(CBr)ccc2ccccc12</smiles>

(R)-2-Bromomethyl-2'-methoxymethoxy-1,1'-binaphthyl (4): $\quad(S)-3$ (3.6 g, $11 \mathrm{mmol}),{ }^{1} \mathrm{~N}$-bromosuccinimide (NBS, $2.1 \mathrm{~g}, 12 \mathrm{mmol}$ ), 2,2'-azobis(isobutyronitrile) $(\mathrm{AIBN}, 0.18 \mathrm{~g}, 1.1 \mathrm{mmol})$ and $\mathrm{CCl}_{4}(100 \mathrm{~mL})$ were placed into flask equipped with reflux condenser. The reaction mixture was stirred under reflux condition for $5 \mathrm{~h}$. After cooling to room temperature, the solvent was removed under reduced pressure. The residue was purified by column chromatography on silica gel (hexane : EtOAc $=20: 1)$ to give $(R)-\mathbf{4}$ as a white solid. $(R)-4$ which contained $(R)$-2-dibromomethyl-2'-methoxymethoxy-1,1'-binaphthyl as a byproduct could be used at the following step, because the dibromide did not react with sodium trifluoromethanesulfinate. TLC, $R_{\mathrm{f}}=0.25$ (hexane : EtOAc $\left.=4: 1\right) ; \mathrm{Mp} .71^{\circ} \mathrm{C} ;[\alpha]^{25.1}{ }_{\mathrm{D}}=70.5(c=$ 0.50, $\mathrm{CHCl}_{3}$ ); IR (KBr) 3053, 2957, 2898, 1507, 1246, 1148, 1067, 1036, 1013, 816, $750 \mathrm{~cm}^{-1} ;{ }^{1} \mathrm{H}$ NMR $\left(\mathrm{CDCl}_{3}, 300 \mathrm{MHz}\right) \delta 3.13(\mathrm{~s}, 3 \mathrm{H}), 4.30(\mathrm{~d}, J=10.0 \mathrm{~Hz}, 1 \mathrm{H}), 4.35(\mathrm{~d}, J=10.0 \mathrm{~Hz}, 1 \mathrm{H}), 4.98$ $(\mathrm{d}, J=6.9 \mathrm{~Hz}, 1 \mathrm{H}), 5.08(\mathrm{~d}, J=6.9 \mathrm{~Hz}, 1 \mathrm{H}), 7.00(\mathrm{~d}, J=8.7 \mathrm{~Hz}, 1 \mathrm{H}), 7.15-7.24(\mathrm{~m}, 3 \mathrm{H}), 7.33(\mathrm{t}, J$ $=7.1 \mathrm{~Hz}, 1 \mathrm{H}), 7.42(\mathrm{~d}, J=7.2 \mathrm{~Hz}, 1 \mathrm{H}), 7.61(\mathrm{~d}, J=9.0 \mathrm{~Hz}, 1 \mathrm{H}), 7.72(\mathrm{~d}, J=8.4 \mathrm{~Hz}, 1 \mathrm{H}), 7.87(\mathrm{~d}, J$ $=7.8 \mathrm{~Hz}, 2 \mathrm{H}), 7.97(\mathrm{~d}, J=9.5 \mathrm{~Hz}, 2 \mathrm{H}) ;{ }^{13} \mathrm{C} \mathrm{NMR}\left(\mathrm{CDCl}_{3}, 75 \mathrm{MHz}\right) \delta 32.6,55.8,94.7,116.2,120.8$, $124.2,125.3,126.2,126.4,126.5,126.6,127.5,127.8,127.9,128.5,129.5,130.0,132.8,133.2$, 133.6, 133.7, 134.0, 152.6. Anal. Calcd for $\mathrm{C}_{23} \mathrm{H}_{19} \mathrm{BrO}_{2}$ : C, 67.82; H, 4.70. Found: C, 67.58; H, 4.60 .<smiles>COc1ccc2ccccc2c1-c1c(C[Tl])ccc2ccccc12</smiles>

(R)-2-Methoxymethoxy-2'-trifluoromethanesulfonylmethyl-1,1'-binap hthyl (5): Sodium trifluoromethanesulfinate $\left(\mathrm{CF}_{3} \mathrm{SO}_{2} \mathrm{Na}, 3.4 \mathrm{~g}, 22 \mathrm{mmol}\right),(R)-4$ (crude, ca. 11 mmol), pyridine $(89 \mu \mathrm{L}, 1.1 \mathrm{mmol})$ and $N, N$ '-dimethylacetamide $(50 \mathrm{~mL})$ were placed into flask equipped with reflux condenser. The reaction mixture was stirred for $12 \mathrm{~h}$ at $100{ }^{\circ} \mathrm{C}$. After cooling to room temperature, the organic layer was washed with $\mathrm{H}_{2} \mathrm{O}$ and brine. The aqueous phase was washed with EtOAc twice. The organic layers were dried over $\mathrm{MgSO}_{4}$, filtered and concentrated. The residue was purified by column chromatography on silica gel (hexane : EtOAc $=20: 1)$ to give $(R)-\mathbf{5}(2.9 \mathrm{~g}, 6.3 \mathrm{mmol})$ as a white solid in $57 \%$ yield from $(S)-\mathbf{3}$. TLC, $R_{\mathrm{f}}=0.30$ 
(hexane : EtOAc $=4: 1) ; \mathrm{Mp} .95^{\circ} \mathrm{C} ;[\alpha]^{22.9}=69.2\left(c=0.44, \mathrm{CHCl}_{3}\right) ; \mathrm{IR}(\mathrm{KBr}) 2957,2929,2902$, $1508,1365,1219,1196,1119,1012,823,753,679,617,476 \mathrm{~cm}^{-1} ;{ }^{1} \mathrm{H} \mathrm{NMR}\left(\mathrm{CDCl}_{3}, 300 \mathrm{MHz}\right) \delta$ $3.09(\mathrm{~s}, 3 \mathrm{H}), 4.32(\mathrm{~d}, J=14.1 \mathrm{~Hz}, 1 \mathrm{H}), 4.43(\mathrm{~d}, J=14.1 \mathrm{~Hz}, 1 \mathrm{H}), 4.87$ (d, $J=7.1 \mathrm{~Hz}, 1 \mathrm{H}), 5.15$ (d, $J=7.1 \mathrm{~Hz}, 1 \mathrm{H}), 6.96(\mathrm{~d}, J=8.4 \mathrm{~Hz}, 1 \mathrm{H}), 7.17-7.25(\mathrm{~m}, 3 \mathrm{H}), 7.33(\mathrm{t}, J=6.9 \mathrm{~Hz}, 1 \mathrm{H}), 7.47$ (quintet, $J=4.0 \mathrm{~Hz}, 1 \mathrm{H}), 7.62(\mathrm{dd}, J=2.1,9.3 \mathrm{~Hz}, 1 \mathrm{H}), 7.78(\mathrm{dd}, J=1.5,8.7 \mathrm{~Hz}, 1 \mathrm{H}), 7.86(\mathrm{~d}, J=8.4 \mathrm{~Hz}$, $1 \mathrm{H}), 7.90(\mathrm{~d}, J=7.8 \mathrm{~Hz}, 1 \mathrm{H}), 7.98(\mathrm{~d}, J=8.7 \mathrm{~Hz}, 2 \mathrm{H}) ;{ }^{13} \mathrm{C} \mathrm{NMR}\left(\mathrm{CDCl}_{3}, 75 \mathrm{MHz}\right) \delta 53,9,55.8$, 94.9, 116.0, 119.4 (q, $\left.J_{\mathrm{CF}}=326 \mathrm{~Hz}, 1 \mathrm{C}\right), 119.6,121.2,124.3,124.9,126.7,126.9,127.0,127.4$,

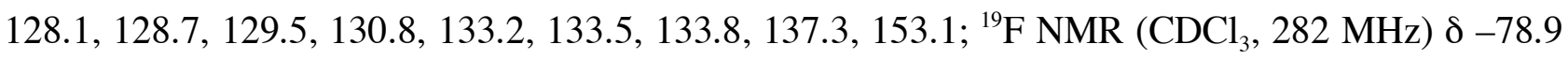
(s, 3F); Anal. Calcd for $\mathrm{C}_{24} \mathrm{H}_{19} \mathrm{~F}_{3} \mathrm{O}_{4} \mathrm{~S}: \mathrm{C}, 62.60 ; \mathrm{H}, 4.16$. Found: C, 62.71; H, 4.04.

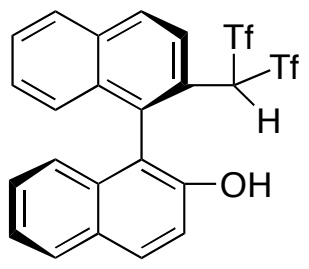

(R)-2-Bis(trifluoromethanesulfonyl)methyl-2'-hydroxy-1,1'-binaphthyl

(1): To a solution of $(R)-5(8.5 \mathrm{~g}, 18.5 \mathrm{mmol})$ in dry DME $(150 \mathrm{~mL})$ was added $n$-BuLi $(12.1 \mathrm{~mL}$, $19.4 \mathrm{mmol}, 1.6 \mathrm{M}$ solution in hexane) dropwise at $-78{ }^{\circ} \mathrm{C}$, and the resulting mixture was stirred for $20 \mathrm{~min}$. Trifluoromethanesulfonic anhydride $(1.9 \mathrm{~mL}, 11.1 \mathrm{mmol})$ was then added, and the resulting mixture was allowed to warm to room temperature over a period of $2 \mathrm{~h}$. After the reaction mixture was cooled to $-78{ }^{\circ} \mathrm{C}, n$-BuLi (12.1 mL, $19.4 \mathrm{mmol}, 1.6 \mathrm{M}$ in hexane) was added dropwise, and the resulting mixture was stirred for $20 \mathrm{~min}$. Trifluoromethanesulfonic anhydride $(1.9 \mathrm{~mL}, 11.1 \mathrm{mmol})$ was then added, and the resulting mixture was allowed to warm to room temperature over a period of $2 \mathrm{~h}$ before the reaction was quenched with water. The resultant mixture was neutralized and washed with hexane. The aqueous phase was acidified with $4 \mathrm{M}$ aqueous $\mathrm{HCl}$ and extracted with ether twice. The organic layers were dried over $\mathrm{MgSO}_{4}$, filtered and concentrated under reduced pressure. The residue was purified by column chromatography on silica gel (hexane : EtOAc $=10: 1$ to EtOAc only) to give $(R)-6$. However, $(R)-6$ was not fully analyzed by ${ }^{1} \mathrm{H}$ NMR and ${ }^{13} \mathrm{C}$ NMR because of its instability: ${ }^{1} \mathrm{H}$ NMR $\left(\mathrm{CD}_{3} \mathrm{OD}, 300 \mathrm{MHz}\right) \delta 3.12$ $(\mathrm{s}, 3 \mathrm{H}), 4.78(\mathrm{~d}, J=8.4 \mathrm{~Hz}, 1 \mathrm{H}), 5.24(\mathrm{~d}, J=8.4 \mathrm{~Hz}, 1 \mathrm{H}), 6.70-7.90(\mathrm{~m}, 12 \mathrm{H})$. Conc. $\mathrm{HCl}(4 \mathrm{~mL})$, $(R)-6(5.1 \mathrm{~g})$ and $\mathrm{MeOH}(50 \mathrm{~mL})$ were placed into flask. The reaction mixture was stirred at $50{ }^{\circ} \mathrm{C}$ for $12 \mathrm{~h}$. After cooling to room temperature, the solvent was removed under reduced pressure. The residue was extracted with ether twice. The organic layers were dried over $\mathrm{MgSO}_{4}$, filtered and concentrated to give $(R)-\mathbf{1}(4.8 \mathrm{~g}, 8.7 \mathrm{mmol})$ in $47 \%$ overall yield from $(R)-\mathbf{5}$. The residual solid was recrystallized from toluene/hexane to give $(R)-\mathbf{1}$. TLC, $R_{\mathrm{f}}=0$ (hexane : EtOAc $=4: 1) ; \mathrm{Mp} .174{ }^{\circ} \mathrm{C} ;[\alpha]_{\mathrm{D}}^{20}=30.6\left(c=1.14, \mathrm{CHCl}_{3}\right)$; IR $(\mathrm{KBr}) 3542,2916,1389,1237,1214,1101$, 834, 668, 641, 594, $503 \mathrm{~cm}^{-1} ;{ }^{1} \mathrm{H} \mathrm{NMR}\left(\mathrm{CDCl}_{3}, 300 \mathrm{MHz}\right) \delta 5.05(\mathrm{~s}, 1 \mathrm{H}, \mathrm{OH}), 6.20(\mathrm{~s}, 1 \mathrm{H}), 6.84(\mathrm{~d}$, $J=8.4 \mathrm{~Hz}, 1 \mathrm{H}), 7.21-7.45(\mathrm{~m}, 5 \mathrm{H}), 7.68(\mathrm{t}, J=7.5 \mathrm{~Hz}, 1 \mathrm{H}), 7.88(\mathrm{t}, J=8.1 \mathrm{~Hz}, 1 \mathrm{H}), 8.00(\mathrm{~d}, J=$ 
$9.3 \mathrm{~Hz}, 1 \mathrm{H}), 8.07(\mathrm{~d}, J=8.1 \mathrm{~Hz}, 1 \mathrm{H}), 8.20(\mathrm{~d}, J=8.1 \mathrm{~Hz}, 1 \mathrm{H}), 8.20(\mathrm{~d}, J=9.0 \mathrm{~Hz}, 1 \mathrm{H}), 8.25(\mathrm{~d}, J=$ $8.7 \mathrm{~Hz}, 1 \mathrm{H}) ;{ }^{13} \mathrm{C} \mathrm{NMR}\left(\mathrm{CDCl}_{3}, 75 \mathrm{MHz}\right) \delta 79.0,112.5,117.9,118.7$ (q, $\left.J_{\mathrm{CF}}=328 \mathrm{~Hz}, 1 \mathrm{C}\right), 119.3$, $119.4\left(\mathrm{q}, J_{\mathrm{CF}}=328 \mathrm{~Hz}, 1 \mathrm{C}\right), 124.3,125.1,125.6,127.3,127.5,128.2,128.4,128.7,128.9,129.3$, 130.9, 132.3, 133.3, 133.4, 135.2, 137.7, 152.4; ${ }^{19} \mathrm{~F}$ NMR $\left(\mathrm{CDCl}_{3}, 282 \mathrm{MHz}\right) \delta-74.7$ (s, 3F), -72.6 (s, 3F). HRMS (FAB+) Calcd for $\mathrm{C}_{23} \mathrm{H}_{14} \mathrm{~F}_{6} \mathrm{O}_{5} \mathrm{~S}_{2}[\mathrm{M}+2 \mathrm{Na}+\mathrm{H}]^{+}:$592.9904, Found 592.9907.

General Procedure for the Preparation of $N$-Benzylidenearylamines (7): Benzaldehyde $(0.3 \mathrm{~mL}, 3 \mathrm{mmol})$ and aniline $(3 \mathrm{mmol})$ were dissolved in ethanol $(10 \mathrm{~mL})$. The reaction mixture was stirred for $1 \mathrm{~h}$ at reflux temperature. After the mixture was cooled to room temperature, the solvent was removed under reduced pressure. Purification of the residue by distillation and/or recrystallization gave $N$-Benzylidenebenzenamine.<smiles>C(=N/c1ccccc1)\c1ccccc1</smiles>

(E)- $N$-Benzylidenebenzenamine (7a): ${ }^{2} \quad$ Mp. $52{ }^{\circ} \mathrm{C}$; IR (KBr) 3650-3150 (br), 3060, 2884, 1626, 1590, 1577, 1483, 1450, 1365, 1193, 1171, $1073 \mathrm{~cm}^{-1} ;{ }^{1} \mathrm{H}$ NMR $\left(\mathrm{CDCl}_{3}, 300\right.$ $\mathrm{MHz}) \delta 7.18-7.30(\mathrm{~m}, 3 \mathrm{H}), 7.35-7.55(\mathrm{~m}, 5 \mathrm{H}), 7.86-7.96(\mathrm{~m}, 2 \mathrm{H}), 8.46(\mathrm{~s}, 1 \mathrm{H}) ;{ }^{13} \mathrm{C} \mathrm{NMR}\left(\mathrm{CDCl}_{3}\right.$, $75 \mathrm{MHz}) \delta 120.8$ (2C), 125.9, 128.7 (2C), 128.8 (2C), 129.1 (2C), 131.3, 136.1, 152.0, 160.4.

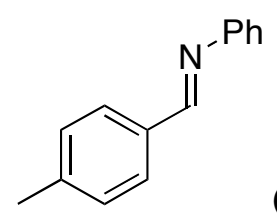

(E)-N-(4-Methylbenzylidene)benzenamine (7b): ${ }^{3} \quad$ Mp. $42{ }^{\circ} \mathrm{C}$; IR $(\mathrm{KBr})$ 3650-3250 (br), 2884, 1623, 1608, 1587, 1483, 1447, 1191, 1170, 1106, $1072 \mathrm{~cm}^{-1}$; ${ }^{1} \mathrm{H}$ NMR $\left(\mathrm{CDCl}_{3}, 300 \mathrm{MHz}\right) \delta 2.36(\mathrm{~s}, 3 \mathrm{H}), 7.16-7.31$ (m, 5H), 7.34-7.43 (m, 2H), 7.76-7.83 (m, 2H), 8.40 $(\mathrm{s}, 1 \mathrm{H}) ;{ }^{13} \mathrm{C} \mathrm{NMR}\left(\mathrm{CDCl}_{3}, 75 \mathrm{MHz}\right) \delta 21.6,120.8$ (2C), 125.7, 128.8 (2C), 129.1 (2C), 129.5 (2C), 133.5, 141.8, 152.1, 160.4;<smiles>Fc1ccccc1/C=N/c1ccccc1</smiles>

(E)-N-(2-Fluorobenzylidene)benzenamine (7c): ${ }^{4}$ IR (film) 3063, 2902, 1703,

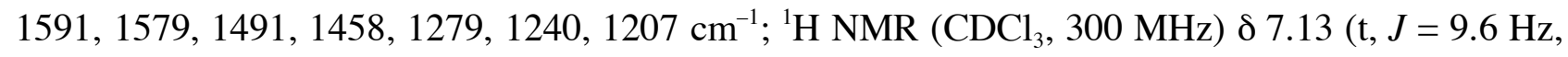
1H), 7.20-7.30 (m, 4H), 7.36-7.51 (m, 3H), 8.19 (dt, $J=1.5,7.5 \mathrm{~Hz}, 1 \mathrm{H}), 8.78(\mathrm{~s}, 1 \mathrm{H}) ;{ }^{13} \mathrm{C}$ NMR $\left(\mathrm{CDCl}_{3}, 125 \mathrm{MHz}\right) \delta 115.7\left(\mathrm{~d}, J_{\mathrm{CF}}=20.5 \mathrm{~Hz}, 1 \mathrm{C}\right), 120.9(2 \mathrm{C}), 123.8\left(\mathrm{~d}, J_{\mathrm{CF}}=9.1 \mathrm{~Hz}, 1 \mathrm{C}\right), 124.3(\mathrm{~d}$, $\left.J_{\mathrm{CF}}=3.0 \mathrm{~Hz}, 1 \mathrm{C}\right), 126.2,127.7\left(\mathrm{~d}, J_{\mathrm{CF}}=2.3 \mathrm{~Hz}, 1 \mathrm{C}\right), 129.0(2 \mathrm{C}), 132.8\left(\mathrm{~d}, J_{\mathrm{CF}}=8.4 \mathrm{~Hz}, 1 \mathrm{C}\right), 151.7$, $153.3\left(\mathrm{~d}, J_{\mathrm{CF}}=4.6 \mathrm{~Hz}, 1 \mathrm{C}\right), 162.7\left(\mathrm{~d}, J_{\mathrm{CF}}=253.0 \mathrm{~Hz}, 1 \mathrm{C}\right)$. 
<smiles>Fc1cccc(/C=N/c2ccccc2)c1</smiles>

(E)- $N$-(3-Fluorobenzylidene)benzenamine (7d): ${ }^{5}$ IR (film) 3066, 2359, 1630, 1585, 1488, 1449, 1366, 1264, 1204, $1135 \mathrm{~cm}^{-1} ;{ }^{1} \mathrm{H}$ NMR $\left(\mathrm{CDCl}_{3}, 300 \mathrm{MHz}\right) \delta 7.13-7.30(\mathrm{~m}$, 4H), 7.35-7.49 (m, 3H), 7.60-7.71 (m, 2H), $8.43(\mathrm{~s}, 1 \mathrm{H}) ;{ }^{13} \mathrm{C} \mathrm{NMR}\left(\mathrm{CDCl}_{3}, 125 \mathrm{MHz}\right) \delta 114.6(\mathrm{~d}$, $\left.J_{\mathrm{CF}}=22.0 \mathrm{~Hz}, 1 \mathrm{C}\right), 118.2\left(\mathrm{~d}, J_{\mathrm{CF}}=22.0 \mathrm{~Hz}, 1 \mathrm{C}\right), 120.8(2 \mathrm{C}), 124.9\left(\mathrm{~d}, J_{\mathrm{CF}}=2.3 \mathrm{~Hz}, 1 \mathrm{C}\right), 126.2$, $129.1(2 \mathrm{C}), 130.2\left(\mathrm{~d}, J_{\mathrm{CF}}=7.5 \mathrm{~Hz}, 1 \mathrm{C}\right), 138.4\left(\mathrm{~d}, J_{\mathrm{CF}}=7.6 \mathrm{~Hz}, 1 \mathrm{C}\right), 151.4,158.7\left(\mathrm{~d}, J_{\mathrm{CF}}=3.1 \mathrm{~Hz}\right.$, 1C), $163.0\left(\mathrm{~d}, J_{\mathrm{CF}}=233.8 \mathrm{~Hz}, 1 \mathrm{C}\right)$.<smiles>Fc1ccc(/C=N/c2ccccc2)cc1</smiles>

(E)-N-[4-(Fluoromethyl)benzylidene]benzenamine $\quad(7 \mathrm{e}): \quad 7 \mathrm{e}$ was purchased from Acros Organics.<smiles>FC(F)(F)c1ccc(/C=N/c2ccccc2)cc1</smiles>

(E)- $N$-[4-(Trifluoromethyl)benzylidene]benzenamine $\quad(\mathbf{7 f}):^{6} \quad \mathrm{Mp}$. $79{ }^{\circ} \mathrm{C}$; IR (KBr) 3650-3275 (br), 2929, 1699, 1623, 1457, 1414, 1323, 1172, 1126, $1066 \mathrm{~cm}^{-1}$; ${ }^{1} \mathrm{H}$ NMR $\left(\mathrm{CDCl}_{3}, 300 \mathrm{MHz}\right)$ d 7.20-7.33 (m, 3H), 7.39-7.46 (m, 2H), $7.74(\mathrm{~d}, J=7.8 \mathrm{~Hz}, 2 \mathrm{H}), 8.03$ (d, $J=7.8 \mathrm{~Hz}, 2 \mathrm{H}), 8.52(\mathrm{~s}, 1 \mathrm{H}) ;{ }^{13} \mathrm{C} \mathrm{NMR}\left(\mathrm{CDCl}_{3}, 125 \mathrm{MHz}\right) \delta 120.6(2 \mathrm{C}), 123.9$ (q, $J_{\mathrm{CF}}=270.8 \mathrm{~Hz}$, $1 C), 125.7\left(\mathrm{q}, J_{\mathrm{CF}}=3.8 \mathrm{~Hz}, 2 \mathrm{C}\right), 126.6,128.9(2 \mathrm{C}), 129.2(2 \mathrm{C}), 132.7\left(\mathrm{q}, J_{\mathrm{CF}}=32.6 \mathrm{~Hz}, 1 \mathrm{C}\right), 139.2$, $151.3,158.5$.<smiles>O=[N+]([O-])c1ccc(/C=N/c2ccccc2)cc1</smiles>

(E)- $N$-(4-Nitrobenzylidene)benzenamine $(7 \mathbf{g}):^{7} \quad 7 \mathrm{~g}$ was prepared by following the known method. ${ }^{7}$

Preparation of $N$-(diarylmethyl)aldimines (10) 


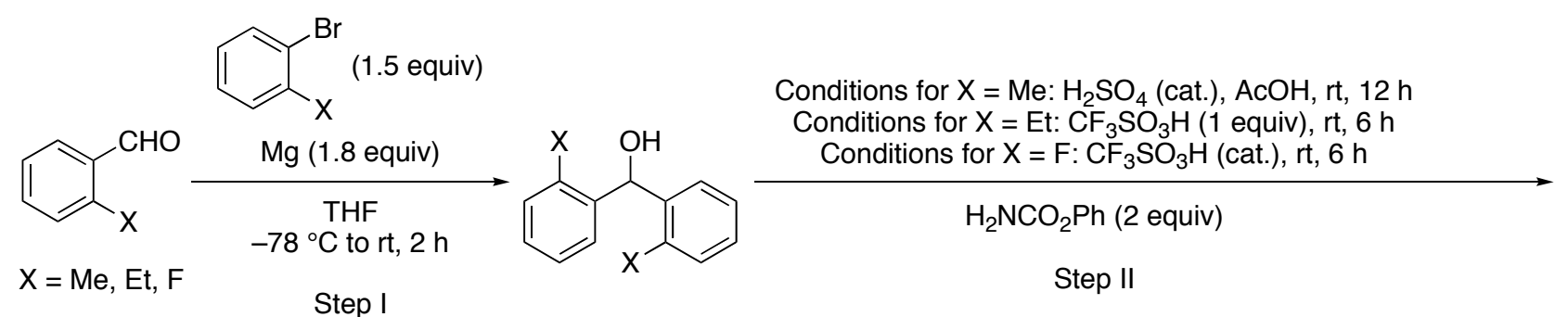<smiles>[X]c1ccccc1C(NC(=O)Oc1ccccc1)c1ccccc1</smiles>
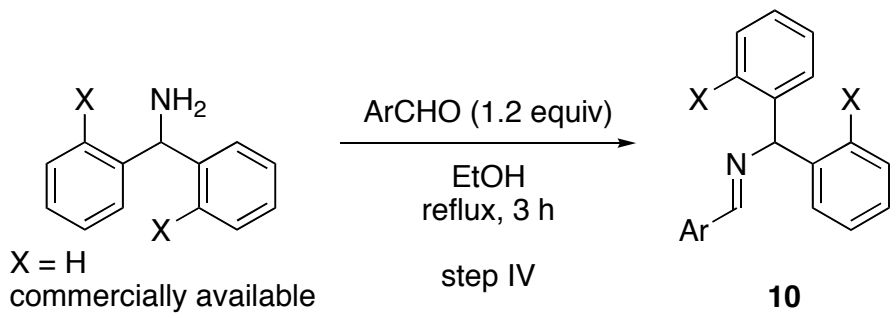

10

Step I. To a mixture of activated magnesium $(18 \mathrm{mmol})$ in dry THF $(15 \mathrm{~mL})$ was added dropwise $o$-bromoarene $(15 \mathrm{mmol})$ over $30 \mathrm{~min}$ at $0{ }^{\circ} \mathrm{C}$. The mixture was stirred at room temperature for $3 \mathrm{~h}$ (For $\mathrm{X}=\mathrm{F}$, Grignard reagent was prepared at $0{ }^{\circ} \mathrm{C}$ for $3 \mathrm{~h}$ ). To a solution of arylaldehyde $(10 \mathrm{mmol})$ in dry THF $(10 \mathrm{mmol})$ was added dropwise the above mixture at $-78{ }^{\circ} \mathrm{C}$. Then, the mixture was stirred at room temperature for $2 \mathrm{~h}$. The reaction was quenched with saturated $\mathrm{NH}_{4} \mathrm{Cl}$ aqueous solution at $0{ }^{\circ} \mathrm{C}$. The product was extracted with EtOAc, washed with $\mathrm{H}_{2} \mathrm{O}$, dried over anhydrous $\mathrm{MgSO}_{4}$, filtered and concentrated in vacuo. The crude product was purified by column chromatography on silica gel (hexane-EtOAc) afforded diarylmethanol.

Step II for $\mathbf{X}=$ Me. According to the procedure by Marchand-Brynaert et al. ${ }^{8}$ a solution of di(o-tolyl)methanol $(0.21 \mathrm{~g}, 1 \mathrm{mmol})$ and phenylcarbamate $(0.27 \mathrm{~g}, 2 \mathrm{mmol})$ in pure acetic acid $(6$ $\mathrm{mL}$ ) containing $2 \%$ of concd $\mathrm{H}_{2} \mathrm{SO}_{4}$ was stirred for $12 \mathrm{~h}$ at room temperature, then poured into ice-cold water $[200 \mathrm{~mL} / \mathrm{g}$ of di(o-tolyl)methanol]. The precipitate was filtered off, dissolved in EtOAc and washed twice with $3 \% \mathrm{NaHCO}_{3}$ aqueous solution. The organic layer was dried over anhydrous $\mathrm{MgSO}_{4}$, filtered and concentrated. The crude product was purified by column chromatography on silica gel (hexane-EtOAc) afforded $N$-[di(o-tolyl)methyl]benzamide.

Step II for $\mathbf{X}=\mathbf{E t}$. A solution of bis(2-ethylphenyl)methanol (0.48 g, $2.0 \mathrm{mmol})$ and phenylcarbamate $(0.56 \mathrm{~g}, 4.0 \mathrm{mmol})$ in pure trifluoroacetic acid $(20 \mu \mathrm{L})$ was stirred at room temperature for $6 \mathrm{~h}$.

Step II for $\mathbf{X}=\mathbf{F}$. A solution of bis(2-fluorophenyl)methanol $(0.22 \mathrm{~g}, 1 \mathrm{mmol})$, phenylcarbamate $(0.27 \mathrm{~g}, 2 \mathrm{mmol})$ and trifluoromethanesulfonic acid $(88 \mu \mathrm{L}, 1 \mathrm{mmol})$ in dry $\mathrm{MeCN}(6 \mathrm{~mL})$ was stirred overnight at room temperature.

Step III. According to the procedure by Marchand-Brynaert et al., ${ }^{8}$ a solution of $N$-(diarylmethyl)benzamide (4.4 mmol) in $\mathrm{MeCN} / \mathrm{H}_{2} \mathrm{O}(50: 50,100 \mathrm{~mL} / \mathrm{g}$ of carbamate) was added $\mathrm{LiOH}\left(40 \mathrm{mmol}\right.$ ) and heated at $50{ }^{\circ} \mathrm{C}$ for $3 \mathrm{~h}$, under stirring. After concentration under vacuum, the residue was dissolved in EtOAc and washed with $\mathrm{H}_{2} \mathrm{O}$. The organic phase was dried over 
$\mathrm{MgSO}_{4}$, filtered, and concentrated in vacuo. The crude product was purified by column chromatography on silica gel (hexane-EtOAc) afforded (diarylmethyl)amine.

Step IV. Arylaldehyde $(3.6 \mathrm{mmol})$ and (diarylmethyl)amine $(3.0 \mathrm{mmol})$ were dissolved in ethanol $(15 \mathrm{~mL})$. The reaction mixture was stirred for $3 \mathrm{~h}$ at reflux temperature. After the mixture was cooled to room temperature, the solvent was removed under reduced pressure. Purification of the residue by distillation and/or recrystallization gave $\mathbf{1 0}$.<smiles>C(=N/C(c1ccccc1)c1ccccc1)\c1ccccc1</smiles>

(E)- $N$-benzylidenediphenylmethanamine $\quad(\mathbf{1 0 a}):{ }^{9} \quad$ Mp. $95{ }^{\circ} \mathrm{C}$; IR $(\mathrm{KBr})$ 3650-3150 (br), 3027, 2925, 2851, 1637, 1492, 1453, 1380, 1277, $1023 \mathrm{~cm}^{-1} ;{ }^{1} \mathrm{H} \mathrm{NMR}\left(\mathrm{CDCl}_{3}, 300\right.$ $\mathrm{MHz}) \delta 5.60(\mathrm{~s}, 1 \mathrm{H}), 7.20-7.50(\mathrm{~m}, 13 \mathrm{H}), 7.82-7.89(\mathrm{~m}, 2 \mathrm{H}), 8.43(\mathrm{~s}, 1 \mathrm{H}) ;{ }^{13} \mathrm{C} \mathrm{NMR}\left(\mathrm{CDCl}_{3}, 75\right.$ MHz) $\delta ~ 77.9,126.9$ (2C), 127.6 (4C), 128.40 (4C), 128.42, 128.5 (2C), 130.7 (2C), 136.2, 143.8 (2C), 160.8 .<smiles>Cc1ccccc1C(=Nc1ccccc1)c1ccccc1C</smiles>

(E)- $N$-benzylidenedi $\left(o\right.$-tolyl)methanamine (10b): $\quad$ Mp. $119{ }^{\circ} \mathrm{C}$; IR $(\mathrm{KBr})$ 3600-3250 (br), 3018, 2860, 1635, 1485, 1459, 1448, 1378, $1021 \mathrm{~cm}^{-1} ;{ }^{1} \mathrm{H}$ NMR $\left(\mathrm{CDCl}_{3}, 300\right.$ MHz) $\delta 2.26(\mathrm{~s}, 6 \mathrm{H}), 6.12(\mathrm{~s}, 1 \mathrm{H}), 7.15-7.30(\mathrm{~m}, 10 \mathrm{H}), 7.36-7.46(\mathrm{~m}, 2 \mathrm{H}), 7.76-7.84(\mathrm{~m}, 1 \mathrm{H}), 8.17$ $(\mathrm{s}, 1 \mathrm{H}) ;{ }^{13} \mathrm{C} \mathrm{NMR}\left(\mathrm{CDCl}_{3}, 75 \mathrm{MHz}\right) \delta 19.4$ (2C), 69.7, 126.0 (2C), 126.9 (2C), 128.3 (2C), 128.4 (2C), 128.5 (2C), 130.3 (2C), 130.7, 135.9 (2C), 136.3, 140.4 (2C), 160.4. HRMS (FAB+) Calcd for $\mathrm{C}_{22} \mathrm{H}_{21} \mathrm{~N}[\mathrm{M}+\mathrm{H}]^{+}: 300.1752$, Found 300.1738 .<smiles>CCc1ccccc1C(/N=C/c1ccccc1)c1ccccc1</smiles>

(E)-N-Benzylidenebis(2-ethylphenyl)methanamine (10c): IR (film) 3062, 3026, 2966, 2932, 2872, 2359, 1642, 1485, 1450, 1375, $1025 \mathrm{~cm}^{-1} ;{ }^{1} \mathrm{H}$ NMR $\left(\mathrm{CDCl}_{3}, 300 \mathrm{MHz}\right) \delta$ $1.15(\mathrm{t}, J=7.5 \mathrm{~Hz}, 6 \mathrm{H}), 2.51-2.74(\mathrm{~m}, 4 \mathrm{H}), 6.26(\mathrm{~s}, 1 \mathrm{H}), 7.15-7.24(\mathrm{~m}, 8 \mathrm{H}), 7.39-7.41(\mathrm{~m}, 3 \mathrm{H})$, 7.77-7.80 (m, 2H), 8.05 (s, 1H); ${ }^{13} \mathrm{C}$ NMR $\left(\mathrm{CDCl}_{3}, 75 \mathrm{MHz}\right) \delta 14.7$ (2C), 25.1 (2C), 68.3, 125.8 (2C), 127.2 (2C), 128.35 (2C), 128.43 (2C), 128.48 (3C), 128.7 (3C), 130.7, 136.3, 139.9, 141.7, 160.6. HRMS (FAB+) Calcd for $\mathrm{C}_{24} \mathrm{H}_{25} \mathrm{~N}[\mathrm{M}+\mathrm{H}]^{+}:$328.2065, Found 328.2056. 
<smiles>Fc1ccccc1C(/N=C/c1ccccc1)c1ccccc1F</smiles>

(E)- $N$-Benzylidenebis(2-fluorophenyl)methanamine (10d): $\quad \mathrm{Mp} .51{ }^{\circ} \mathrm{C}$;

IR (KBr) 3304, 2853, 1644, 1585, 1487, 1451, 1384, 1225, 1100, $1030 \mathrm{~cm}^{-1} ;{ }^{1} \mathrm{H}$ NMR $\left(\mathrm{CDCl}_{3}, 300\right.$ MHz) $\delta 6.29(\mathrm{~s}, 1 \mathrm{H}), 6.98-7.08(\mathrm{~m}, 2 \mathrm{H}), 7.10-7.17(\mathrm{~m}, 2 \mathrm{H}), 7.20-7.31(\mathrm{~m}, 3 \mathrm{H}), 7.41$ (d, J = 2.1 Hz, $1 \mathrm{H}), 7.43(\mathrm{~d}, J=2.1 \mathrm{~Hz}, 1 \mathrm{H}), 7.48-7.56(\mathrm{~m}, 2 \mathrm{H}), 7.80-7.85(\mathrm{~m}, 2 \mathrm{H}), 8.46(\mathrm{~s}, 1 \mathrm{H}) ;{ }^{13} \mathrm{C}$ NMR $\left(\mathrm{CDCl}_{3}, 125 \mathrm{MHz}\right) \delta 63.1,115.2\left(\mathrm{~d}, J_{\mathrm{CF}}=21.3 \mathrm{~Hz}, 2 \mathrm{C}\right), 124.0(2 \mathrm{C}), 128.4\left(\mathrm{~d}, J_{\mathrm{CF}}=5.4 \mathrm{~Hz}, 4 \mathrm{C}\right)$, $128.7\left(\mathrm{~d}, J_{\mathrm{CF}}=7.6 \mathrm{~Hz}, 2 \mathrm{C}\right), 128.8,129.3(2 \mathrm{C}), 129.6130 .9,136.0,160.0\left(\mathrm{~d}, J_{\mathrm{CF}}=245.6 \mathrm{~Hz}, 2 \mathrm{C}\right)$, 162.0. HRMS (FAB+) Calcd for $\mathrm{C}_{20} \mathrm{H}_{15} \mathrm{~F}_{2} \mathrm{~N}[\mathrm{M}+\mathrm{H}]^{+}$: 308.1251 Found 308.1236.<smiles>Fc1ccc(/C=N/C(c2ccccc2F)c2ccccc2F)cc1</smiles>

(E)- $N$-(4-Fluorobenzylidene)bis(2-fluorophenyl)methanamine

(10e): Mp. $59{ }^{\circ} \mathrm{C}$; IR (KBr) 2926, 2855, 1637, 1599, 1508, 1487, 1456, 1225, 1151, $1092 \mathrm{~cm}^{-1}$; ${ }^{1} \mathrm{H} \mathrm{NMR}\left(\mathrm{CDCl}_{3}, 300 \mathrm{MHz}\right) \delta 6.27(\mathrm{~s}, 1 \mathrm{H}), 6.98-7.16(\mathrm{~m}, 6 \mathrm{H}), 7.21-7.32(\mathrm{~m}, 2 \mathrm{H}), 7.46-7.54(\mathrm{~m}$, 2H), 7.79-7.86 (m, 2H), $8.42(\mathrm{~s}, 1 \mathrm{H}) ;{ }^{13} \mathrm{C} \mathrm{NMR}\left(\mathrm{CDCl}_{3}, 125 \mathrm{MHz}\right) \delta 63.1,115.4\left(\mathrm{~d}, J_{\mathrm{CF}}=21.1 \mathrm{~Hz}\right.$, 2C), $115.6\left(\mathrm{~d}, J_{\mathrm{CF}}=22.0 \mathrm{~Hz}, 2 \mathrm{C}\right), 124.1(2 \mathrm{C}), 128.8\left(\mathrm{~d}, J_{\mathrm{CF}}=8.3 \mathrm{~Hz}, 2 \mathrm{C}\right), 129.3\left(\mathrm{~d}, J_{\mathrm{CF}}=3.1 \mathrm{~Hz}\right.$, 2C), $129.4\left(\mathrm{~d}, J_{\mathrm{CF}}=13.6 \mathrm{~Hz}, 2 \mathrm{C}\right), 130.4\left(\mathrm{~d}, J_{\mathrm{CF}}=9.1 \mathrm{~Hz}, 2 \mathrm{C}\right), 132.4\left(\mathrm{~d}, J_{\mathrm{CF}}=3.0 \mathrm{~Hz}, 1 \mathrm{C}\right), 160.1(\mathrm{~d}$, $\left.J_{\mathrm{CF}}=245.6 \mathrm{~Hz}, 2 \mathrm{C}\right), 160.7 .164 .5\left(\mathrm{~d}, J_{\mathrm{CF}}=249.5 \mathrm{~Hz}, 1 \mathrm{C}\right)$. HRMS (FAB+) Calcd for $\mathrm{C}_{20} \mathrm{H}_{14} \mathrm{~F}_{3} \mathrm{~N}$ $[\mathrm{M}+\mathrm{H}]^{+}: 326.1157$ Found 326.1168.<smiles>CCOC(OC)=C(C)C</smiles>

Preparation of Triethyl(1-methoxy-2-methylprop-1-enyloxy)silane $(8 \mathrm{~b}):^{10}$ According to the procedure by Shibasaki et al., ${ }^{11}$ to a solution of $i-\mathrm{Pr}_{2} \mathrm{NH}(5.1 \mathrm{~mL}, 36 \mathrm{mmol})$ in THF (35 mL), BuLi (1.6 M solution in hexane, $20.6 \mathrm{~mL}, 33 \mathrm{mmol})$ was added dropwise in an ice bath, and the whole was stirred for $15 \mathrm{~min}$. After cooling to $-78^{\circ} \mathrm{C}$, a mixture of methyl isobutyrate $(3.4 \mathrm{~mL}, 30 \mathrm{mmol})$ and $\mathrm{Et}_{3} \mathrm{SiCl}(6.0 \mathrm{~mL}, 36 \mathrm{mmol})$ in THF $(18 \mathrm{~mL})$ was added. After the addition was completed, the cooling bath was removed, and the mixture was stirred for $3 \mathrm{~h}$ at room temperature. Then, THF was evaporated and hexane was added. The resulting precipitate was filtrated off through a celite pad, and the residue was washed with hexane. The combined filtrate 
was evaporated, and the resulting residue was purified by distillation, giving product in $72 \%$ yield. IR (film) 2956, 2927, 2870, 1705, 1456, 1205, 1176, 1146, $1065 \mathrm{~cm}^{-1} ;{ }^{1} \mathrm{H} \mathrm{NMR}\left(\mathrm{CDCl}_{3}, 300 \mathrm{MHz}\right)$ $\delta 0.69$ (q, $J=7.8 \mathrm{~Hz}, 6 \mathrm{H}), 0.99(\mathrm{t}, J=7.8 \mathrm{~Hz}, 9 \mathrm{H}), 1.54(\mathrm{~s}, 3 \mathrm{H}), 1.56(\mathrm{~s}, 3 \mathrm{H}), 3.52(\mathrm{~s} .3 \mathrm{H}) ;{ }^{13} \mathrm{C} \mathrm{NMR}$ $\left(\mathrm{CDCl}_{3}, 75 \mathrm{MHz}\right) \delta 4.9(3 \mathrm{C}), 6.6(3 \mathrm{C}), 16.2,16.8,57.1,91.0,149.7$.

\section{General Procedure for the Enantioselective Mannich-type Reaction Catalyzed by $(R)-1$}

(Tables 1 and 2): To a solution of $(R)-1(0.025 \mathrm{mmol})$, aldimine (7 or 10, $0.25 \mathrm{mmol})$ and alcohol ( $t$-BuOH or 2,6-xylenol, $0.25 \mathrm{mmol})$ in 1-chloropropane $(1.5 \mathrm{~mL})$ was added dropwise ketene silyl acetal $(8,0.375 \mathrm{mmol})$ at $-78{ }^{\circ} \mathrm{C}$. The reaction mixture was stirred for $24 \mathrm{~h}$ at the same temperature. The reaction was quenched with $\mathrm{Et}_{3} \mathrm{~N}$ ( 2 drops) and the mixture was allowed to warm up to room temperature. The resultant mixture was directly done by column chromatography on silica gel (hexane-EtOAc) to give product 9 or $\mathbf{1 1}$. The enantiomeric excess was determined by HPLC.

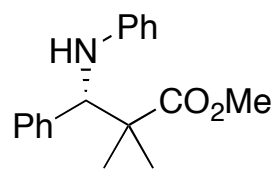

(S)-Methyl 2,2-dimethyl-3-phenyl-3-(phenylamino)propanoate $(9 a):^{12}$ Mp. $102{ }^{\circ} \mathrm{C},[\alpha]_{\mathrm{D}}^{23.0}=-1.4\left(c=4.49, \mathrm{CHCl}_{3}\right)$ for $42 \%$ ee; Mp. $102{ }^{\circ} \mathrm{C}$; IR $(\mathrm{KBr}) 3630-3150$ (br), 1715, 1602, 1508, 1497, 1469, 1431, 1309, 1281, 1251, 1187, $1135 \mathrm{~cm}^{-1} ;{ }^{1} \mathrm{H}$ NMR $\left(\mathrm{CDCl}_{3}, 300\right.$ MHz) $\delta 1.16(\mathrm{~s}, 3 \mathrm{H}), 1.27(\mathrm{~s}, 3 \mathrm{H}), 3.66(\mathrm{~s}, 3 \mathrm{H}), 4.49(\mathrm{~d}, J=7.8 \mathrm{~Hz}, 1 \mathrm{H}), 4.80(\mathrm{~d}, J=7.8 \mathrm{~Hz}, 1 \mathrm{H})$, 6.46-6.53 (m, 2H), 6.56-6.64 (m, 1H), 7.00-7.08 (m, 2H), 7.18-7.33 (m, 5H); ${ }^{13} \mathrm{C} \mathrm{NMR}\left(\mathrm{CDCl}_{3}\right.$, $75 \mathrm{MHz}) \delta$ 20.6, 24.5, 46.9, 52.0, 64.2, 113.3 (2C), 117.2, 127.7, 127.9 (2C), 128.2 (2C), 128.9 (2C), 139.1, 146.8, 177.0; The ee was determined by HPLC analysis (Daicel Chiralpak AS-H column, hexane- $i-\mathrm{PrOH}=80: 1$ for elution, flow rate $=0.5 \mathrm{~mL} / \mathrm{min} ; \lambda=244 \mathrm{~nm}) t_{\mathrm{R}}=12.8$ $((S)$-enantiomer), $15.2((R)$-enantiomer) min. The absolute configuration of $9 \mathbf{a}$ was determined by comparison of the CD-spectra of 2,2-dimethyl-3-phenyl-3-(phenylamino)propan-1-ol derived by reduction of $9 \mathbf{a}$ with $\mathrm{LiAlH}_{4}$ with that in the literature. ${ }^{13}$

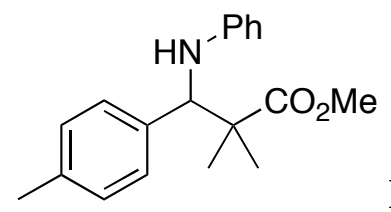

Methyl 2,2-Dimethyl-3-(phenylamino)-3-p-tolylpropanoate $(9 \mathrm{~b}):^{14}$

Mp. $76^{\circ} \mathrm{C}$; IR (KBr) 3650-3200 (br), 2925, 2860, 2361, 2343, 1718, 1603, 1508, 1458, 1437, 1251, $1137 \mathrm{~cm}^{-1} ;{ }^{1} \mathrm{H}$ NMR $\left(\mathrm{CDCl}_{3}, 300 \mathrm{MHz}\right) \delta 1.15$ (s, 3H), 1.26 (s, 3H), 2.29 (s, 3H), 3.65 (s, 3H), 4.46 $(\mathrm{d}, J=6.3 \mathrm{~Hz}, 1 \mathrm{H}), 4.62(\mathrm{~d}, J=6.3 \mathrm{~Hz}, 1 \mathrm{H}), 6.42-6.66(\mathrm{~m}, 3 \mathrm{H}), 6.93-7.23(\mathrm{~m}, 6 \mathrm{H}) ;{ }^{13} \mathrm{C} \mathrm{NMR}$ $\left(\mathrm{CDCl}_{3}, 75 \mathrm{MHz}\right) \delta$ 20.6, 21.0, 24.5, 47.0, 52.0, 64.0, 113.3 (2C), 117.1, 128.1 (2C), 128.7 (2C), 128.9 (2C), 136.1, 136.9, 147.0, 177.1; The ee was determined by HPLC analysis (Daicel 
Chiralpak AD-H column, hexane- $i-\mathrm{PrOH}=80: 1$ for elution, flow rate $=0.5 \mathrm{~mL} / \mathrm{min} ; \lambda=244 \mathrm{~nm}$ ) $t_{\mathrm{R}}=13.0$ (major enantiomer), 14.8 (minor enantiomer) min.<smiles>CC(=O)C(C)(C)C(Nc1ccccc1)c1ccccc1F</smiles>

Methyl

3-(2-Fluorophenyl)-2,2-dimethyl-3-(phenylamino)propanoate $(9 \mathrm{c}): \quad \mathrm{Mp} .79{ }^{\circ} \mathrm{C} ;[\alpha]^{24.4}=19.3$ $\left(c=2.96, \mathrm{CHCl}_{3}\right)$ for $69 \%$ ee; IR (KBr) 3650-3250 (br), 1712, 1603, 1509, 1490, 1469, 1280, 1253, 1220, $1140 \mathrm{~cm}^{-1} ;{ }^{1} \mathrm{H}$ NMR $\left(\mathrm{CDCl}_{3}, 300 \mathrm{MHz}\right) \delta 1.19$ (s, 3H), 1.33 (s, 3H), 3.67 (s, 3H), 4.87-5.00 (m, 2H), 6.50-6.56 (m, 2H), 6.59-6.65 (m, 1H), 6.96-7.11 (m, 4H), 7.16-7.24 (m, 2H); ${ }^{13} \mathrm{C}$ NMR $\left(\mathrm{CDCl}_{3}, 125 \mathrm{MHz}\right) \delta 20.5,24.6,47.2,52.1,57.2,113.1(2 \mathrm{C}), 115.2\left(\mathrm{~d}, J_{\mathrm{CF}}=22.8 \mathrm{~Hz}, 1 \mathrm{C}\right), 117.5$, $124.0\left(\mathrm{~d}, J_{\mathrm{CF}}=3.0 \mathrm{~Hz}, 1 \mathrm{C}\right), 126.6\left(\mathrm{~d}, J_{\mathrm{CF}}=12.9 \mathrm{~Hz}, 1 \mathrm{C}\right), 128.9,129.0,129.1(2 \mathrm{C}), 146.6,161.3(\mathrm{~d}$, $\left.J_{\mathrm{CF}}=243.5 \mathrm{~Hz}, 1 \mathrm{C}\right), 176.8$. HRMS (FAB+) Calcd for $\mathrm{C}_{18} \mathrm{H}_{20} \mathrm{FNO}_{2}[\mathrm{M}+\mathrm{H}]^{+}:$302.1556, Found 302.1556. The ee was determined by HPLC analysis (Daicel Chiralpak AS-H column, hexane- $i-\mathrm{PrOH}=80: 1$ for elution, flow rate $=0.5 \mathrm{~mL} / \mathrm{min} ; \lambda=244 \mathrm{~nm}$ ) $t_{\mathrm{R}}=13.1$ (major enantiomer), 20.4 (minor enantiomer) $\min$.<smiles>CC(=O)C(C)(C)C(Nc1ccccc1)c1cccc(F)c1</smiles>

Methyl

3-(3-Fluorophenyl)-2,2-dimethyl-3-(phenylamino)propanoate (9d): $\mathrm{Mp} .94{ }^{\circ} \mathrm{C} ;[\alpha]^{22.8}{ }_{\mathrm{D}}=-2.0$ $\left(c=4.11, \mathrm{CHCl}_{3}\right)$ for $69 \%$ ee; IR $(\mathrm{KBr}) 3373,1718,1604,1507,1470,1447,1432,1275,1250$, 1189, $1137 \mathrm{~cm}^{-1} ;{ }^{1} \mathrm{H}$ NMR $\left(\mathrm{CDCl}_{3}, 300 \mathrm{MHz}\right) \delta 1.17(\mathrm{~s}, 3 \mathrm{H}), 1.29(\mathrm{~s}, 3 \mathrm{H}), 3.66(\mathrm{~s}, 3 \mathrm{H}), 4.47(\mathrm{~d}, J=$ $7.5 \mathrm{~Hz}, 1 \mathrm{H}), 4.80(\mathrm{~d}, J=7.5 \mathrm{~Hz}, 1 \mathrm{H}), 6.44-6.53(\mathrm{~m}, 2 \mathrm{H}), 6.59-6.67(\mathrm{~m}, 1 \mathrm{H}), 6.88-7.12(\mathrm{~m}, 5 \mathrm{H})$, 7.21-7.31 (m, 1H); ${ }^{13} \mathrm{C}$ NMR $\left(\mathrm{CDCl}_{3}, 125 \mathrm{MHz}\right) \delta 20.8,24.4,46.9,52.1,64.0,113.3(2 \mathrm{C}), 114.4(\mathrm{~d}$, $\left.J_{\mathrm{CF}}=21.3 \mathrm{~Hz}, 1 \mathrm{C}\right), 115.0\left(\mathrm{~d}, J_{\mathrm{CF}}=22.0 \mathrm{~Hz}, 1 \mathrm{C}\right), 117.5,124.0\left(\mathrm{~d}, J_{\mathrm{CF}}=3.0 \mathrm{~Hz}, 1 \mathrm{C}\right), 129.0(2 \mathrm{C})$, $129.4\left(\mathrm{~d}, J_{\mathrm{CF}}=8.4 \mathrm{~Hz}, 1 \mathrm{C}\right), 142.3\left(\mathrm{~d}, J_{\mathrm{CF}}=6.1 \mathrm{~Hz}, 1 \mathrm{C}\right) 146.6,162.7\left(\mathrm{~d}, J_{\mathrm{CF}}=244.9 \mathrm{~Hz}, 1 \mathrm{C}\right), 176.7$. HRMS (FAB+) Calcd for $\mathrm{C}_{18} \mathrm{H}_{20} \mathrm{FNO}_{2}[\mathrm{M}+\mathrm{H}]^{+}$: 302.1556, Found 302.1556. The ee was determined by HPLC analysis (Daicel Chiralpak AS-H column, hexane- $i$-PrOH $=80: 1$ for elution, flow rate $=0.5 \mathrm{~mL} / \mathrm{min} ; \lambda=244 \mathrm{~nm}$ ) $t_{\mathrm{R}}=15.7$ (major enantiomer), 19.4 (minor enantiomer) $\min$.<smiles>CC(=O)C(C)(C)C(Nc1ccccc1)c1ccc(F)cc1</smiles>

Methyl

3-(4-Fluorophenyl)-2,2-dimethyl-3-(phenylamino)propanoate $(9 \mathrm{e}): \quad \mathrm{Mp} .69^{\circ} \mathrm{C} ;[\alpha]^{22.9}{ }_{\mathrm{D}}=-10.3$ 
$\left(c=3.33, \mathrm{CHCl}_{3}\right)$ for $72 \%$ ee; IR $(\mathrm{KBr}) 3374,2991,1714,1604,1508,1469,1432,1250,1226$, $1137 \mathrm{~cm}^{-1} ;{ }^{1} \mathrm{H}$ NMR $\left(\mathrm{CDCl}_{3}, 300 \mathrm{MHz}\right) \delta 1.15(\mathrm{~s}, 3 \mathrm{H}), 1.27(\mathrm{~s}, 3 \mathrm{H}), 3.65(\mathrm{~s}, 3 \mathrm{H}), 4.46(\mathrm{~d}, J=6.9$ $\mathrm{Hz}, 1 \mathrm{H}), 4.78(\mathrm{~d}, J=6.9 \mathrm{~Hz}, 1 \mathrm{H}), 6.44-6.50(\mathrm{~m}, 2 \mathrm{H}), 6.58-6.65(\mathrm{~m}, 1 \mathrm{H}), 6.93-7.09(\mathrm{~m}, 4 \mathrm{H})$, 7.21-7.29 (m, 2H); ${ }^{13} \mathrm{C}$ NMR $\left(\mathrm{CDCl}_{3}, 75 \mathrm{MHz}\right) \delta 20.7,24.4,46.9,52.1,63.7,113.3(2 \mathrm{C}), 114.9(\mathrm{~d}$, $\left.J_{\mathrm{CF}}=2.1 \mathrm{~Hz}, 2 \mathrm{C}\right), 117.4,129.0(2 \mathrm{C}), 129.7\left(\mathrm{~d}, J_{\mathrm{CF}}=8.0 \mathrm{~Hz}, 2 \mathrm{C}\right), 135.0,146.6,162.1\left(\mathrm{~d}, J_{\mathrm{CF}}=244.1\right.$ $\mathrm{Hz}, 1 \mathrm{C}), 176.8$. HRMS (FAB+) Calcd for $\mathrm{C}_{18} \mathrm{H}_{20} \mathrm{FNO}_{2}[\mathrm{M}+\mathrm{H}]^{+}:$302.1556, Found 302.1562. The ee was determined by HPLC analysis (Daicel Chiralpak AS-H column, hexane- $i$-PrOH = $80: 1$ for elution, flow rate $=0.5 \mathrm{~mL} / \mathrm{min} ; \lambda=244 \mathrm{~nm}$ ) $t_{\mathrm{R}}=20.7$ (major enantiomer), 35.5 (minor enantiomer) $\mathrm{min}$.<smiles>CC(=O)C(C)(C)C(Nc1ccccc1)c1ccc(C(F)(F)F)cc1</smiles>

Methyl

2,2-Dimethyl-3-(phenylamino)-3-(4-(trifluoromethyl)phenyl)propanoate (9f): ${ }^{14} \mathrm{Mp} .80{ }^{\circ} \mathrm{C}$; IR (KBr) 3362, 2991, 2950, 1714, 1604, 1326, 1254, 1166, 1142, 1121, $1066 \mathrm{~cm}^{-1} ;{ }^{1} \mathrm{H}$ NMR $\left(\mathrm{CDCl}_{3}\right.$, $300 \mathrm{MHz}) \delta 1.17$ (s, 3H), 1.30 (s, 3H), 3.67 (s, 3H), 4.54 (d, $J=7.2 \mathrm{~Hz}, 1 \mathrm{H}), 4.85$ (d, $J=7.2 \mathrm{~Hz}$, 1H), 6.42-6.56 (m, 2H), 6.60-6.70 (m, 2H), 7.00-7.18 (m, 2H), $7.42(\mathrm{~d}, J=8.1 \mathrm{~Hz}, 2 \mathrm{H}), 7.55(\mathrm{~d}, J$ $=8.1 \mathrm{~Hz}, 2 \mathrm{H}) ;{ }^{13} \mathrm{C} \mathrm{NMR}\left(\mathrm{CDCl}_{3}, 125 \mathrm{MHz}\right) \delta 20.8,24.4,46.8,52.2,64.1,113.3(2 \mathrm{C}), 117.7,124.1$ $\left(\mathrm{q}, J_{\mathrm{CF}}=270.0 \mathrm{~Hz}, 1 \mathrm{C}\right), 125.0\left(\mathrm{q}, J_{\mathrm{CF}}=3.8 \mathrm{~Hz}, 2 \mathrm{C}\right), 128.6(2 \mathrm{C}), 129.1(2 \mathrm{C}), 129.7\left(\mathrm{q}, J_{\mathrm{CF}}=32.0 \mathrm{~Hz}\right.$, 1C), 143.7, 146.4, 176.6. The ee was determined by HPLC analysis (Daicel Chiralcel OD-H column, hexane- $i-\mathrm{PrOH}=400: 1$ for elution, flow rate $=1.0 \mathrm{~mL} / \mathrm{min} ; \lambda=244 \mathrm{~nm}$ ) $t_{\mathrm{R}}=20.1$ (major enantiomer), 23.5 (minor enantiomer) min.<smiles>CC(=O)C(C)(C)C(Nc1ccccc1)c1ccc([N+](=O)[O-])cc1</smiles>

Methyl

2,2-Dimethyl-3-(phenylamino)-3-(4-nitrophenyl)propanoate (9g): ${ }^{15} \quad{ }^{1} \mathrm{H} \quad \mathrm{NMR} \quad\left(\mathrm{CDCl}_{3}, \quad 300\right.$ $\mathrm{MHz}) \delta 1.18$ (s, 3H), 1.32 (s, 3H), 3.67 (s, 3H), 4.58 (s, 1H), 4.80-5.00 (br, 1H), 6.45 (d, J = 8.4 Hz, $2 \mathrm{H}), 6.64(\mathrm{t}, J=7.2 \mathrm{~Hz}, 1 \mathrm{H}), 7.06(\mathrm{~d}, J=8.1 \mathrm{~Hz}, 2 \mathrm{H}), 7.49(\mathrm{~d}, J=8.7 \mathrm{~Hz}, 2 \mathrm{H}), 8.16(\mathrm{~d}, J=8.7 \mathrm{~Hz}$, $2 \mathrm{H}) ;{ }^{13} \mathrm{C} \mathrm{NMR}\left(\mathrm{CDCl}_{3}, 75 \mathrm{MHz}\right) \delta 21.1,24.4,46.7,52.3,64.1,113.3$ (2C), 117.9, 123.3 (2C), 129.1 (2C), 129.2 (2C), 146.1, 147.3, 147.5, 176.2. The ee was determined by HPLC analysis (Daicel Chiralpak AD column, hexane $-i$-PrOH $=80: 1$ for elution, flow rate $=1.0 \mathrm{~mL} / \mathrm{min} ; \lambda=244 \mathrm{~nm}) t_{\mathrm{R}}$ $=34.2$ (minor enantiomer), 39.4 (major enantiomer) $\mathrm{min}$. 
<smiles>COC(C)(C)C(C)(C)OC(C)(C)c1ccccc1</smiles>

(S)-Methyl 3-(Benzhydrylamino)-2,2-dimethyl-3-phenylpropanoate (11a): ${ }^{16,17}$ Hydrochloride: $[\alpha]^{21.2}=-13.7(c=0.22,1 \mathrm{M} \mathrm{HCl})\left(\right.$ lit. $^{18}[\alpha]_{\mathrm{D}}^{23}=-32.8(c=1.1,1 \mathrm{M}$ $\mathrm{HCl})$ ); IR (KBr) 3650-3150 (br), 2982, 2939, 1737, 1245, 1191, $1131 \mathrm{~cm}^{-1} ;{ }^{1} \mathrm{H}$ NMR $\left(\mathrm{CDCl}_{3}, 300\right.$ $\mathrm{MHz}) \delta 1.03$ (s, 3H), 1.13 (s, 3H), $2.36(\mathrm{~s}, 1 \mathrm{H}), 3.66$ (s, 3H), 3.74 (s, 1H), $4.44(\mathrm{~s}, 1 \mathrm{H}), 7.12-7.37$ (m, 15H); ${ }^{13} \mathrm{C} \mathrm{NMR}\left(\mathrm{CDCl}_{3}, 75 \mathrm{MHz}\right) \delta 19.6,24.2,47.2$, 51.6, 63.7, 65.7, 126.9, 127.0 (3C), 127.4, 127.87 (3C), 127.93 (2C), 128.2 (2C), 128.4 (2C), 129.0, 138.8, 143.0, 144.7, 177.4. The ee was determined by HPLC analysis (Daicel Chiralpak AD column, hexane- $i$-PrOH $=400: 1$ for elution, flow rate $=0.5 \mathrm{~mL} / \mathrm{min} ; \lambda=210 \mathrm{~nm}) t_{\mathrm{R}}=19.7((R)$-enantiomer $), 20.6((S)$-enantiomer $)$ min. The absolute configuration of 11a was determined by comparison of the optical rotation of $N$-free amino ester with that in the literature. ${ }^{18} \quad \mathbf{1 1 a}(18 \mathrm{mg}, 0.048 \mathrm{mmol})$ and palladium-activated carbon $(\mathrm{Pd}$ $10 \%, 4 \mathrm{mg})$ and $\mathrm{MeOH}(5 \mathrm{~mL})$ were charged into $100 \mathrm{~mL}$-flask carefully. The reaction mixture was stirred for $3 \mathrm{~h}$ at room temperature under $\mathrm{H}_{2}$ atmosphere. The resultant mixture was filtered through Celite ${ }^{\circledR}$ carefully. The filtrate was concentrated under reduced pressure. The residue was purified by column chromatography on NH silica gel (hexane : EtOAc $=20: 1-2: 1$ ) to give free amine. ${ }^{1} \mathrm{H}$ and ${ }^{13} \mathrm{C}$ NMR spectroscopic properties were identical to those reported in the literature. $^{19}$

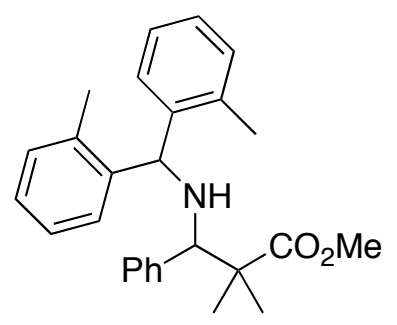

Methyl

3-(Bis(2-methylphenyl)methylamino)-2,2-dimethyl-3-phenylpropanoate $(11 b):[\alpha]_{\mathrm{D}}^{23.4}=-2.1$ $\left(c=2.22, \mathrm{CHCl}_{3}\right.$ ) for 69\% ee; Mp. $141^{\circ} \mathrm{C}$; IR (KBr) 3650-3250 (br), 2979, 2939, 1731, 1491, 1460, 1427, 1387, 1247, 1190, $1132 \mathrm{~cm}^{-1} ;{ }^{1} \mathrm{H}$ NMR $\left(\mathrm{CDCl}_{3}, 300 \mathrm{MHz}\right) \delta 1.05$ (s, 3H), 1.15 (s, 3H), 1.80 (s, 3H), 2.06 (s, 3H), 3.65 (s, 3H), 3.77 (s, 1H), 4.82 (s, 1H), 7.00-7.17 (m, 8H), 7.23-7.37 (m, 5H), $7.66(\mathrm{~d}, J=8.1 \mathrm{~Hz}, 1 \mathrm{H}) ;{ }^{13} \mathrm{C} \mathrm{NMR}\left(\mathrm{CDCl}_{3}, 75 \mathrm{MHz}\right) \delta 19.2,19.4,20.1,24.4,47.2,51.7,55.4,66.0$, 125.5, 126.0, 126.4, 126.6, 127.5, 127.9 (2C), 128.2, 128.4, 129.1 (2C), 130.2, 130.3, 135.7, 136.3, 139.5, 139.9, 141.4, 177.6. HRMS (FAB+) Calcd for $\mathrm{C}_{27} \mathrm{H}_{31} \mathrm{NO}_{2}[\mathrm{M}+\mathrm{H}]^{+}:$402.2433, Found 402.2443. The ee was determined by HPLC analysis (Daicel Chiralpak AD column, hexane- $i-\mathrm{PrOH}=400: 1$ for elution, flow rate $=0.5 \mathrm{~mL} / \mathrm{min} ; \lambda=210 \mathrm{~nm}) t_{\mathrm{R}}=6.1$ (minor enantiomer), 6.6 (major enantiomer) min. 


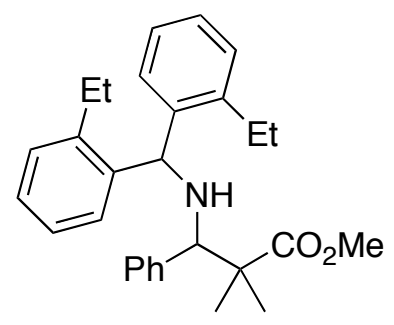

Methyl

3-(Bis(2-ethylphenyl)methylamino)-2,2-dimethyl-3-phenylpropanoate $(11 c): \quad[\alpha]_{\mathrm{D}}^{23.9}=15.2(c$ $=1.00, \mathrm{CHCl}_{3}$ ) for 87\% ee; IR (film) 3062, 3026, 2968, 2933, 2873, 1733, 1486, 1454, 1249, 1192, $1133 \mathrm{~cm}^{-1} ;{ }^{1} \mathrm{H} \mathrm{NMR}\left(\mathrm{CDCl}_{3}, 300 \mathrm{MHz}\right) \delta 0.70(\mathrm{t}, J=7.8 \mathrm{~Hz}, 3 \mathrm{H}), 1.05(\mathrm{~s}, 3 \mathrm{H}), 1.11(\mathrm{t}, J=7.8 \mathrm{~Hz}$, $3 \mathrm{H}), 1.16(\mathrm{~s}, 3 \mathrm{H}), 1.96-2.22(\mathrm{~m}, 2 \mathrm{H}), 2.40-2.60(\mathrm{~m}, 2 \mathrm{H}), 3.67(\mathrm{~s}, 3 \mathrm{H}), 3.83(\mathrm{~s}, 3 \mathrm{H}), 7.02-7.33(\mathrm{~m}$, 12H), $7.72(\mathrm{~d}, J=7.5 \mathrm{~Hz}, 1 \mathrm{H}) ;{ }^{13} \mathrm{C} \mathrm{NMR}\left(\mathrm{CDCl}_{3}, 125 \mathrm{MHz}\right) \delta 14.3,15.2,20.3,24.30,24.33,24.8$, 47.2, 51.7, 54.7, 66.2, 125.3, 125.7, 126.6, 126.9, 127.5, 127.8 (2C), 128.27, 128.30, 128.5, 128.8, 129.3 (2C), 139.1, 139.4, 140.9, 141.7, 142.2, 177.6. HRMS (FAB+) Calcd for $\mathrm{C}_{29} \mathrm{H}_{35} \mathrm{NO}_{2}[\mathrm{M}+\mathrm{H}]^{+}$: 430.2746, Found 430.2734. The ee was determined by HPLC analysis (two linear Daicel Chiralcel OD-H columns, hexane $-i$-PrOH $=400: 1$ for elution, flow rate $=0.5 \mathrm{~mL} / \mathrm{min} ; \lambda=210 \mathrm{~nm}) t_{\mathrm{R}}=$ 27.9 (minor enantiomer), 30.5 (major enantiomer) min.

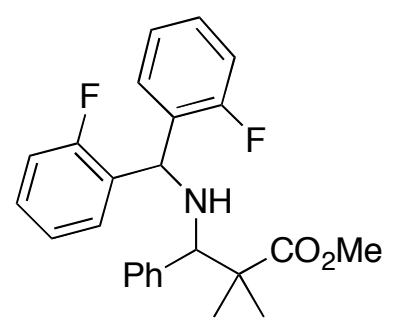

\section{Methyl}

3-(Bis(2-fluorophenyl)methylamino)-2,2-dimethyl-3-phenylpropanoate (11d): $[\alpha]_{D}^{24.3}=-36.2$ $\left(c=3.97, \mathrm{CHCl}_{3}\right)$ for $65 \%$ ee; Mp. $85^{\circ} \mathrm{C}$; IR $(\mathrm{KBr}) 3316,1720,1488,1456,1281,1251,1226$, 1192, 1135, $1100 \mathrm{~cm}^{-1} ;{ }^{1} \mathrm{H}$ NMR $\left(\mathrm{CDCl}_{3}, 300 \mathrm{MHz}\right) \delta 1.01(\mathrm{~s}, 3 \mathrm{H}), 1.14(\mathrm{~s}, 3 \mathrm{H}), 2.38(\mathrm{~s}, 1 \mathrm{H}), 3.64$ (s, 3H), 3.79 (s, 1H), $5.08(\mathrm{~s}, 1 \mathrm{H}), 6.86-6.98(\mathrm{~m}, 2 \mathrm{H}), 7.04-7.35(\mathrm{~m}, 9 \mathrm{H}), 7.38-7.51(\mathrm{~m}, 2 \mathrm{H}) ;{ }^{13} \mathrm{C}$ $\operatorname{NMR}\left(\mathrm{CDCl}_{3}, 125 \mathrm{MHz}\right) \delta 19.4,24.3,47.2,50.6,51.6,66.0,115.2\left(\mathrm{~d}, J_{\mathrm{CF}}=12.9 \mathrm{~Hz}, 1 \mathrm{C}\right), 115.4(\mathrm{~d}$, $\left.J_{\mathrm{CF}}=13.6 \mathrm{~Hz}, 1 \mathrm{C}\right), 123.9,124.0,127.5,127.8(2 \mathrm{C}), 128.3,128.4\left(\mathrm{~d}, J_{\mathrm{CF}}=13.0 \mathrm{~Hz}, 1 \mathrm{C}\right), 128.5(\mathrm{~d}$, $\left.J_{\mathrm{CF}}=12.9 \mathrm{~Hz}, 1 \mathrm{C}\right), 128.7\left(\mathrm{~d}, J_{\mathrm{CF}}=12.9 \mathrm{~Hz}, 1 \mathrm{C}\right), 129.0(2 \mathrm{C}), 129.6,130.2\left(\mathrm{~d}, J_{\mathrm{CF}}=12.9 \mathrm{~Hz}, 1 \mathrm{C}\right)$, $138.3,159.4\left(\mathrm{~d}, J_{\mathrm{CF}}=113.8 \mathrm{~Hz}, 1 \mathrm{C}\right), 161.4\left(\mathrm{~d}, J_{\mathrm{CF}}=113.8 \mathrm{~Hz}, 1 \mathrm{C}\right), 177.4$. HRMS (FAB+) Calcd for $\mathrm{C}_{25} \mathrm{H}_{25} \mathrm{~F}_{2} \mathrm{NO}_{2}[\mathrm{M}+\mathrm{H}]^{+}: 410.1932$ Found 410.1940. The ee was determined by HPLC analysis (Daicel Chiralpak AD-H column, hexane- $i-\mathrm{PrOH}=400: 1$ for elution, flow rate $=0.5 \mathrm{~mL} / \mathrm{min} ; \lambda=$ $210 \mathrm{~nm}) t_{\mathrm{R}}=31.0$ (minor enantiomer), 33.5 (major enantiomer) min. 


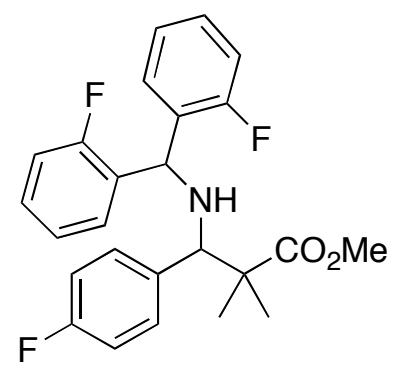

\section{Methyl}

3-(Bis(2-fluorophenyl)methylamino)-3-(4-fluorophenyl)-2,2-dimethylpropanoate

(11e): $[\alpha]_{\mathrm{D}}^{23.3}=-33.4\left(c=5.37, \mathrm{CHCl}_{3}\right)$ for $73 \%$ ee; Mp. $89^{\circ} \mathrm{C}$; IR $(\mathrm{KBr}) 3319,1726,1509,1489,1457$, 1278, 1249, 1223, 1134, $1106 \mathrm{~cm}^{-1} ;{ }^{1} \mathrm{H}$ NMR $\left(\mathrm{CDCl}_{3}, 300 \mathrm{MHz}\right) \delta 1.01$ (s, 3H), 1.13 (s, 3H), 2.35 $(\mathrm{s}, 1 \mathrm{H}), 3.63(\mathrm{~s}, 3 \mathrm{H}), 3.77(\mathrm{~s}, 1 \mathrm{H}), 5.04(\mathrm{~s}, 1 \mathrm{H}), 6.87-7.27(\mathrm{~m}, 10 \mathrm{H}), 7.36-7.47(\mathrm{~m}, 2 \mathrm{H}) ;{ }^{13} \mathrm{C} \mathrm{NMR}$ $\left(\mathrm{CDCl}_{3}, 125 \mathrm{MHz}\right) \delta 19.5,24.1,47.2,50.7,51.7,65.4,114.7\left(\mathrm{~d}, J_{\mathrm{CF}}=20.5 \mathrm{~Hz}, 2 \mathrm{C}\right), 115.2\left(\mathrm{~d}, J_{\mathrm{CF}}=\right.$ $16.0 \mathrm{~Hz}, 1 \mathrm{C}), 115.4\left(\mathrm{~d}, J_{\mathrm{CF}}=16.0 \mathrm{~Hz}, 1 \mathrm{C}\right), 123.9\left(\mathrm{~d}, J_{\mathrm{CF}}=13.0 \mathrm{~Hz}, 2 \mathrm{C}\right), 128.3,128.58\left(\mathrm{~d}, J_{\mathrm{CF}}=\right.$ $12.9 \mathrm{~Hz}, 2 \mathrm{C}), 128.64\left(\mathrm{~d}, J_{\mathrm{CF}}=14.4 \mathrm{~Hz}, 1 \mathrm{C}\right), 129.5,130.1\left(\mathrm{~d}, J_{\mathrm{CF}}=13.6 \mathrm{~Hz}, 1 \mathrm{C}\right), 130.5\left(\mathrm{~d}, J_{\mathrm{CF}}=7.6\right.$ $\mathrm{Hz}, 2 \mathrm{C}), 134.1,159.9$ (d, $\left.J_{\mathrm{CF}}=245.8 \mathrm{~Hz}, 1 \mathrm{C}\right), 160.8$ (q, $\left.J_{\mathrm{CF}}=245.8 \mathrm{~Hz}, 1 \mathrm{C}\right), 162.2\left(\mathrm{~d}, J_{\mathrm{CF}}=244.1\right.$ $\mathrm{Hz}, 1 \mathrm{C})$, 177.3. HRMS (FAB+) Calcd for $\mathrm{C}_{25} \mathrm{H}_{24} \mathrm{~F}_{3} \mathrm{NO}_{2}[\mathrm{M}+\mathrm{H}]^{+}:$428.1837, Found 428.1829. The ee was determined by HPLC analysis (Daicel Chiralpak AD-H column, hexane- $i$-PrOH $=400: 1$ for elution, flow rate $=0.5 \mathrm{~mL} / \mathrm{min} ; \lambda=210 \mathrm{~nm}$ ) $t_{\mathrm{R}}=14.5$ (minor enantiomer), 18.5 (major enantiomer) $\min$.

Crystal data for $(\boldsymbol{R})$-1: Bruker SMART APEX diffractometer with CCD detector (graphite monochromator, Mo K $\alpha$ radiation, $\lambda=0.71073 \AA$ ). The structure was solved by direct methods and expanded using Fourier techniques. Formula $\left(\mathrm{C}_{23} \mathrm{H}_{14} \mathrm{~F}_{6} \mathrm{O}_{5} \mathrm{~S}_{2}\right)_{8}$, colorless, crystal dimensions $0.30 \times 0.30 \times 0.25 \mathrm{~mm}^{3}$, triclinic, space group $P 1, a=8.0791(18) \AA, b=15.672(3) \AA, c$ $=36.082(8) \AA, \beta=90.015(4)^{\circ}, V=4568.6(17) \AA^{3}, Z=1$, and $D_{\text {calc }}=1.595 \mathrm{~g} \mathrm{~cm}^{-3}, \mathrm{~F}(000)=2224, \mu$ $=0.317 \mathrm{~mm}^{-1}, T=223(2) \mathrm{K} . \quad 34484$ reflections collected, 27861 independent reflections with $I>$ $2 \sigma(I)\left(2 \theta_{\max }=29.19^{\circ}\right)$, and 2601 parameters were used for the solution of the structure. $R_{1}=$ 0.0521 and $w R 2=0.1254, \mathrm{GOF}=1.023$. Crystallographic data (excluding structure factors) for the structure reported in this paper have been deposited with Cambridge Crystallographic Data Centre. Copies of the data can be obtained free of charge on application to CCDC, 12 Union Road, Cambridge CB2 1EZ, UK [Fax: int. code + 44(1223)336-033; E-mail: deposit@ccdc.cam.ac.uk]. Supplementary publication no. CCDC-261872. 


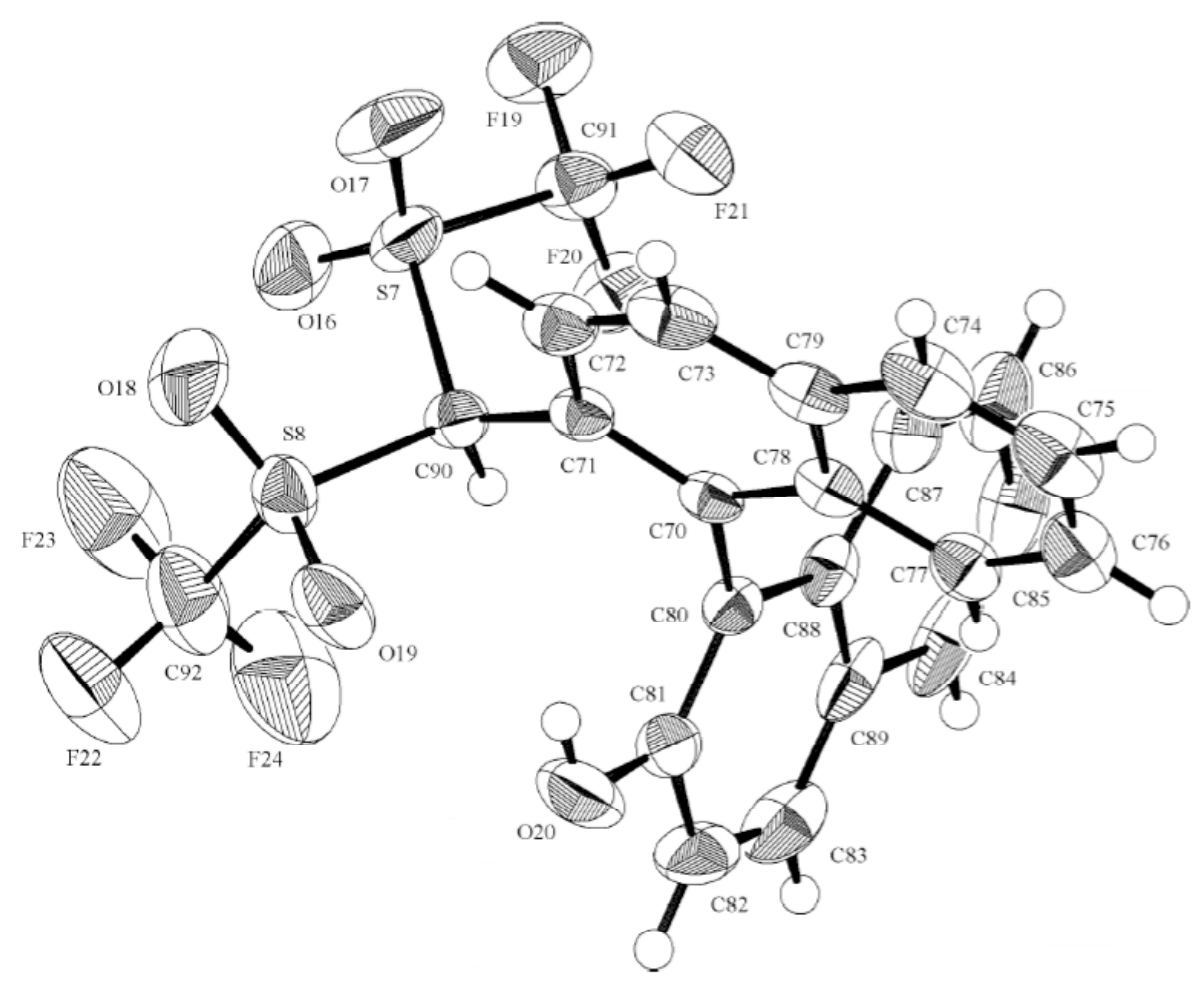

\section{References}

1. Hosoya, N.; Hatayama, A.; Irie, R.; Sasaki, H.; Katsuki, T. Tetrahedron 1994, 50, 4311.

2. Gluchowski, C.; Cooper, L.; Bergbreiter, D. E.; Newcomb, M. J. Org. Chem. 1980, 45, 3413.

3. Xu, H.-W.; Li, G.-Y.; Wong, M.-K.; Che, C.-M. Org. Lett. 2005, 7, 5349.

4. Paventi, M.; Hay, A. S. Tetrahedron Lett. 1993, 34, 999.

5. Toullec, J.; Bennour, S. J. Org. Chem. 1994, 59, 2831.

6. Neuvonen, K.; Fulop, F.; Neuvonen, H.; Koch, A.; Kleinpeter, E.; Pihlaja, K. J. Org. Chem. 2003, 68, 2151.

7. Miecznikowski, J. R.; Crabtree, R. H. Polyhedron 2004, 23, 2857.

8. Laurent, M.; Marchand-Brynaert, J.; Synthesis 2000, 667.

9. Lautens, M.; Tayama, E.; Nguyen, D. Org. Lett. 2004, 6, 345.

10. Takeda, K.; Shibata, Y.; Sagawa, Y.; Urahata, M.; Funaki, K.; Hori, K.; Sasahara, H.; Yoshii, E. J. Org. Chem. 1985, 50, 4673.

11. Oisaki, K.; Suto, Y.; Kanai, M.; Shibasaki, M. J. Am. Chem. Soc. 2003, 125, 5644.

12. Takeuchi, M.; Akiyama, R.; Kobayashi, S. J. Am. Chem. Soc. 2005, 127, 13096.

13. Berova, N.; Christoskova, S.; Ivanov, P.; Kurtev, B.; Simova, E.; Snatzke, G.; Krüger, C. Tetrahedron: Asymmetry 1992, 3, 651.

14. Matsukawa, S.; Obu, K. Chem. Lett. 2004, 33, 1626. 
15. Shi, M.; Cui, S.-C.; Liu, Y.-H. Tetrahedron 2005, 61, 4965.

16. Mukaiyama, T.; Kashiwagi, K.; Matsui, S. Chem. Lett. 1989, 1397.

17. Soga, T.; Takenoshita, H.; Yamada, M.; Mukaiyama, T. Bull. Chem. Soc. Jpn. 1990, 63, 3122 .

18. Kunz, H.; Schanzenbach, D. Angew. Chem. Int. Ed. 1989, 28, 1068.

19. Kobayashi, S.; Ishitani, H.; Yamashita, Y.; Ueno, M.; Shimizu, H.; Tetrahedron 2001, 57, 861. 


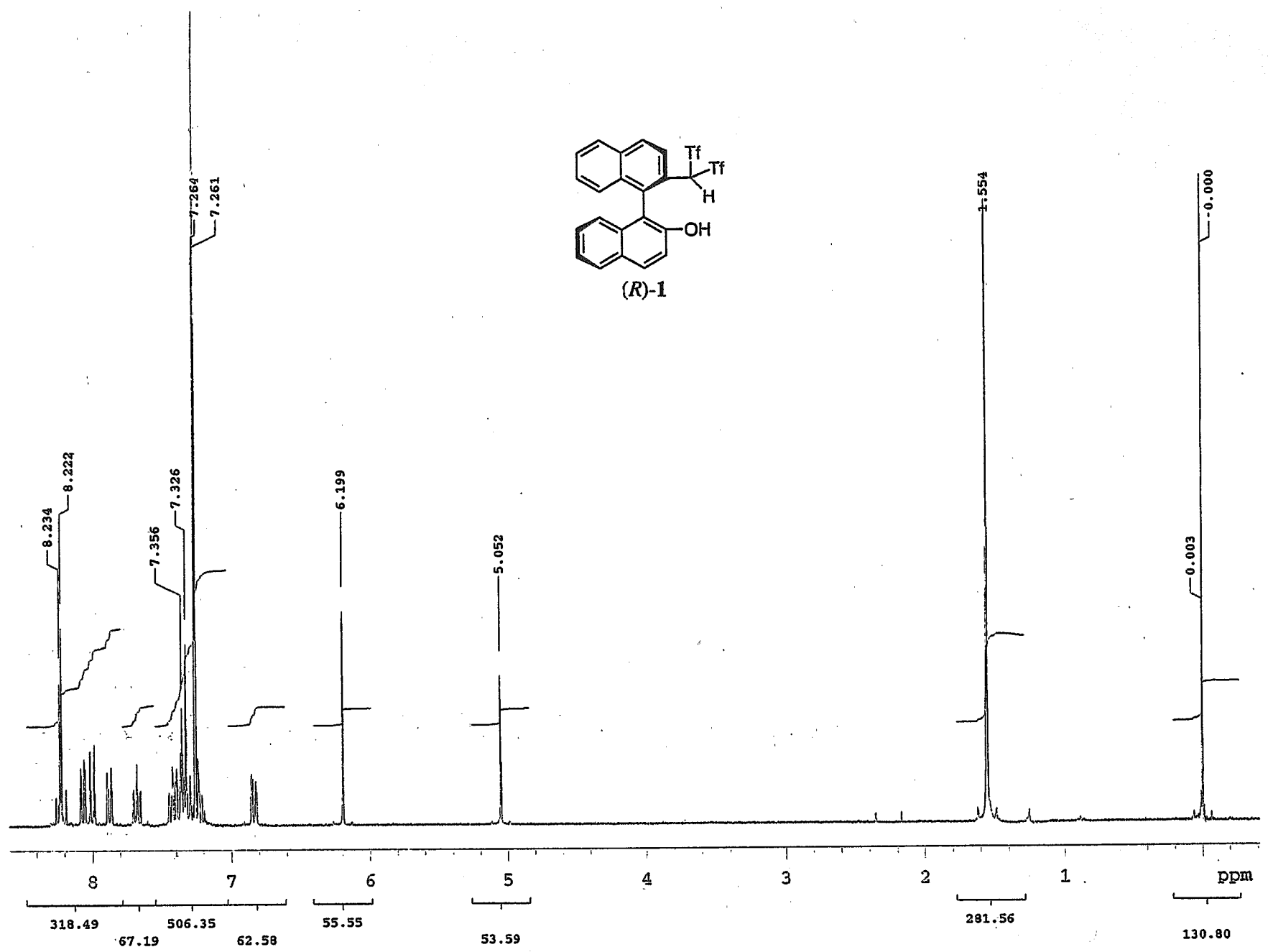

Tfe2, $0 \mathrm{H}$

Pulse Sequence: s2pul

SoIvent: CDC13

Ambient temperature

GEMINI-300BB "varian"

Pu1se 45.0 degrees

Acq. time 1.815 sec

width $18761.7 \mathrm{gz}$

640 cepetitions

ORSERVE C13，75.4528126 $\mathrm{MHz}$

DECOUPIE HI, $300.0720883 \mathrm{MHZ}$

Power $33 \mathrm{~dB}$

continuousiy on

WALT2-16 modulated

DATA PROCESSING

Iine broadening $1.0 \mathrm{~Hz}$

FT size 131072

Total time $65 \mathrm{hr}, 58 \mathrm{~min}, 35 \mathrm{sec}$
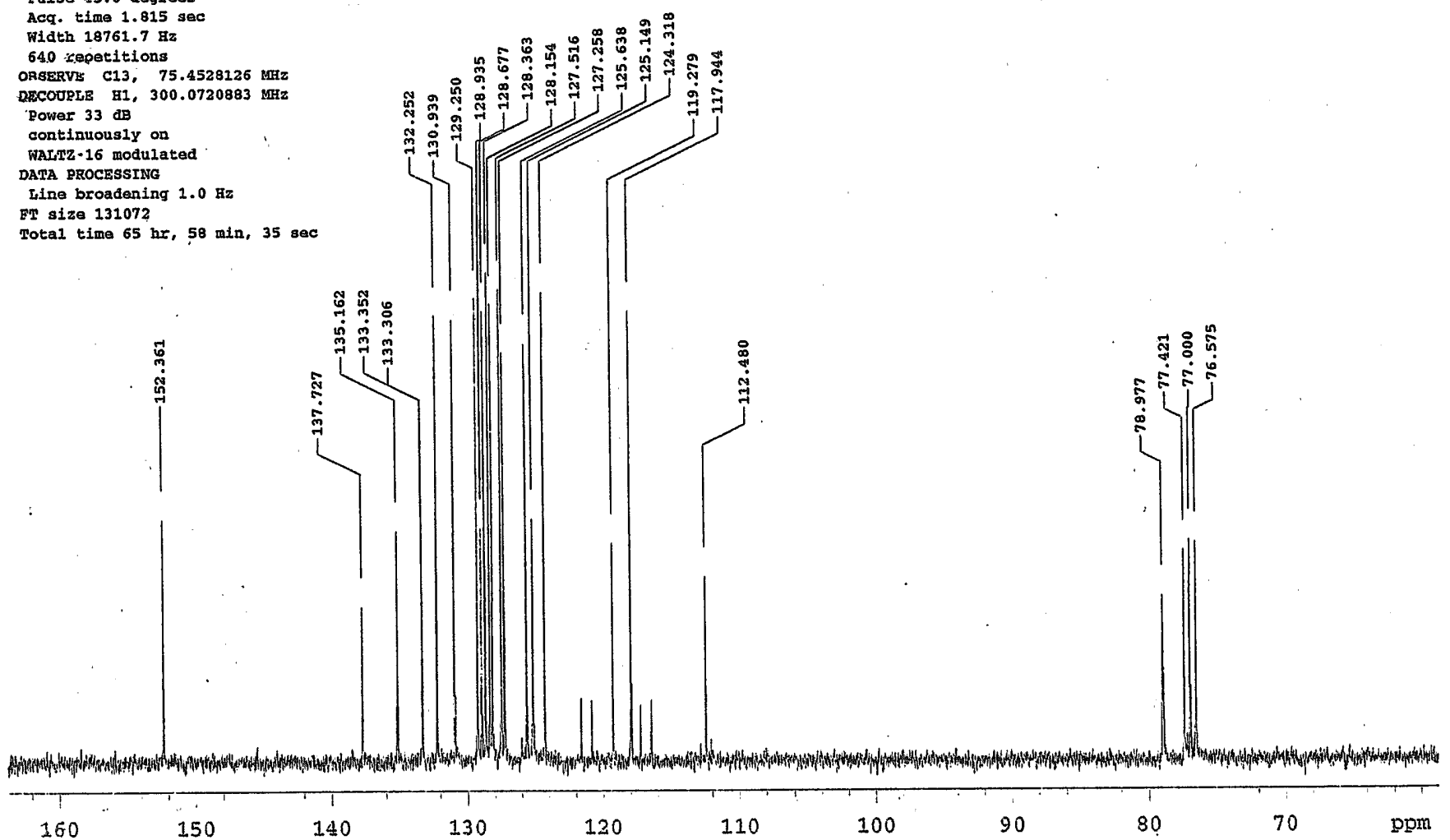
Pulse Sequence: s2pul

Solvent: $\operatorname{CDC} 13$

Amblent temperature

GEMINI-300BB "rarian

Relax. delay $1.000 \mathrm{sec}$

Pulse 45.0 degrees

Acq. time $1.998 \mathrm{sec}$

Width $4500.5 \mathrm{Ez}$

16 repetitions

OBSERVE H1, $300.0706045 \mathrm{MHZ}$

DATA PROCEssING

FT size 32768

Total time $0 \mathrm{~min}, 49 \mathrm{sec}$
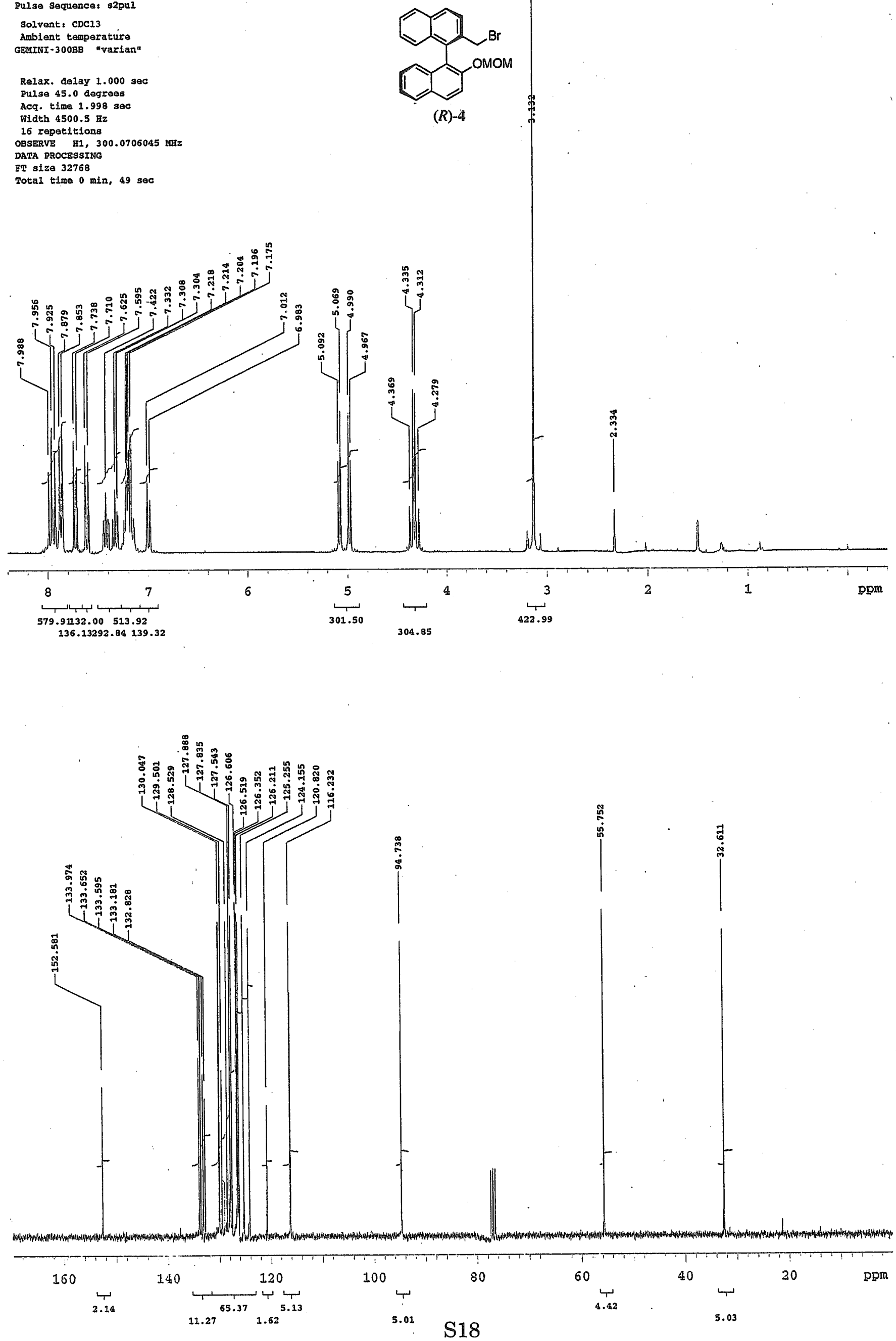


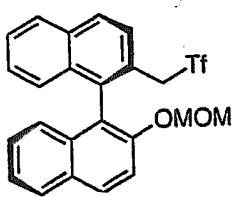

$(R)-5$

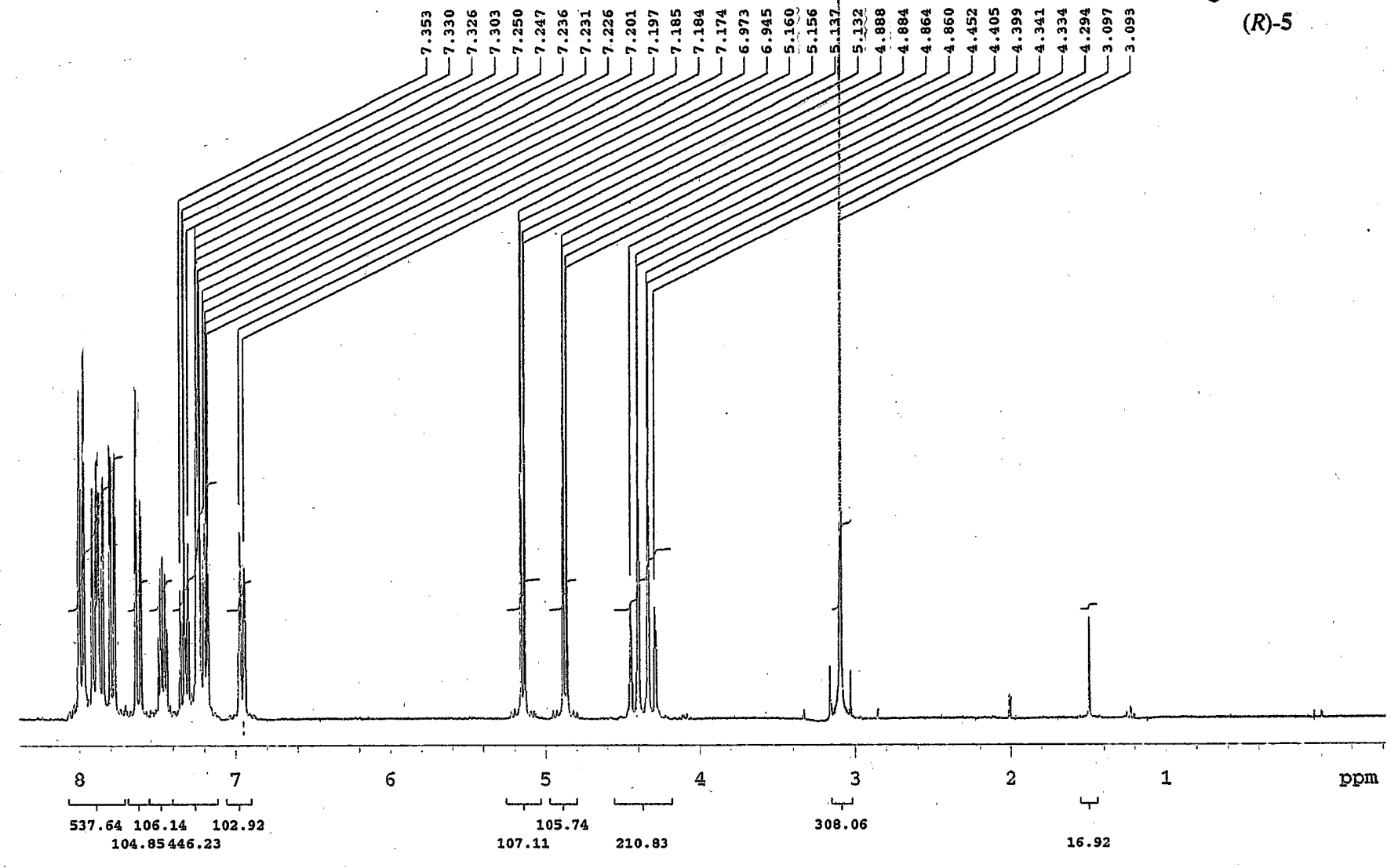

TE, OMOM

Pulse Sequence: s2pul

Solvent: CDC13

Ambient temperature

GEMINI-300BB "varian"

Pulse 45.0 degrees

Acq. timo $1.815 \mathrm{sec}$

Width $18761.7 \mathrm{~Hz}$

696 repatitions

OBSERVE C13, 75.4528172 MHF

DECOUPLE H1, 300.0720883 BHFZ

Power $33 \mathrm{~dB}$

continuousiy on

WALTZ - 16 modula

Iine broadening $1.0 \mathrm{~Hz}$

FT size 131072

Total time $6597 \mathrm{hr}, 38$ min.

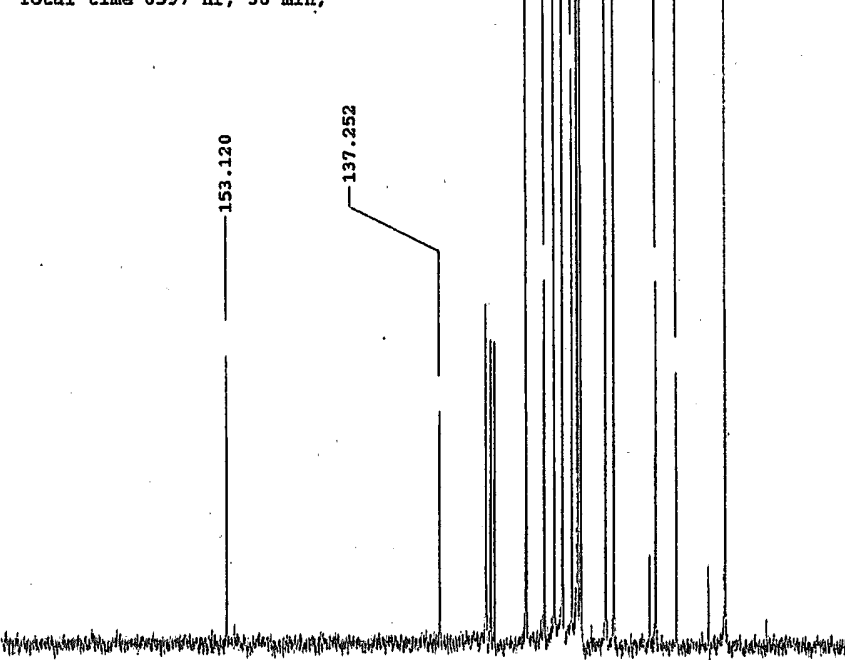

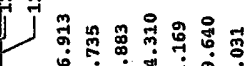

政

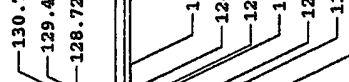

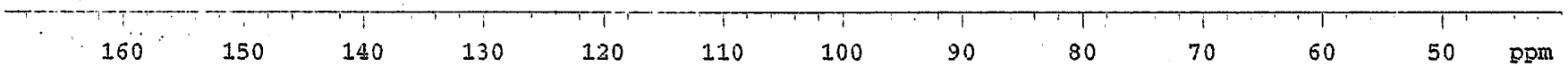



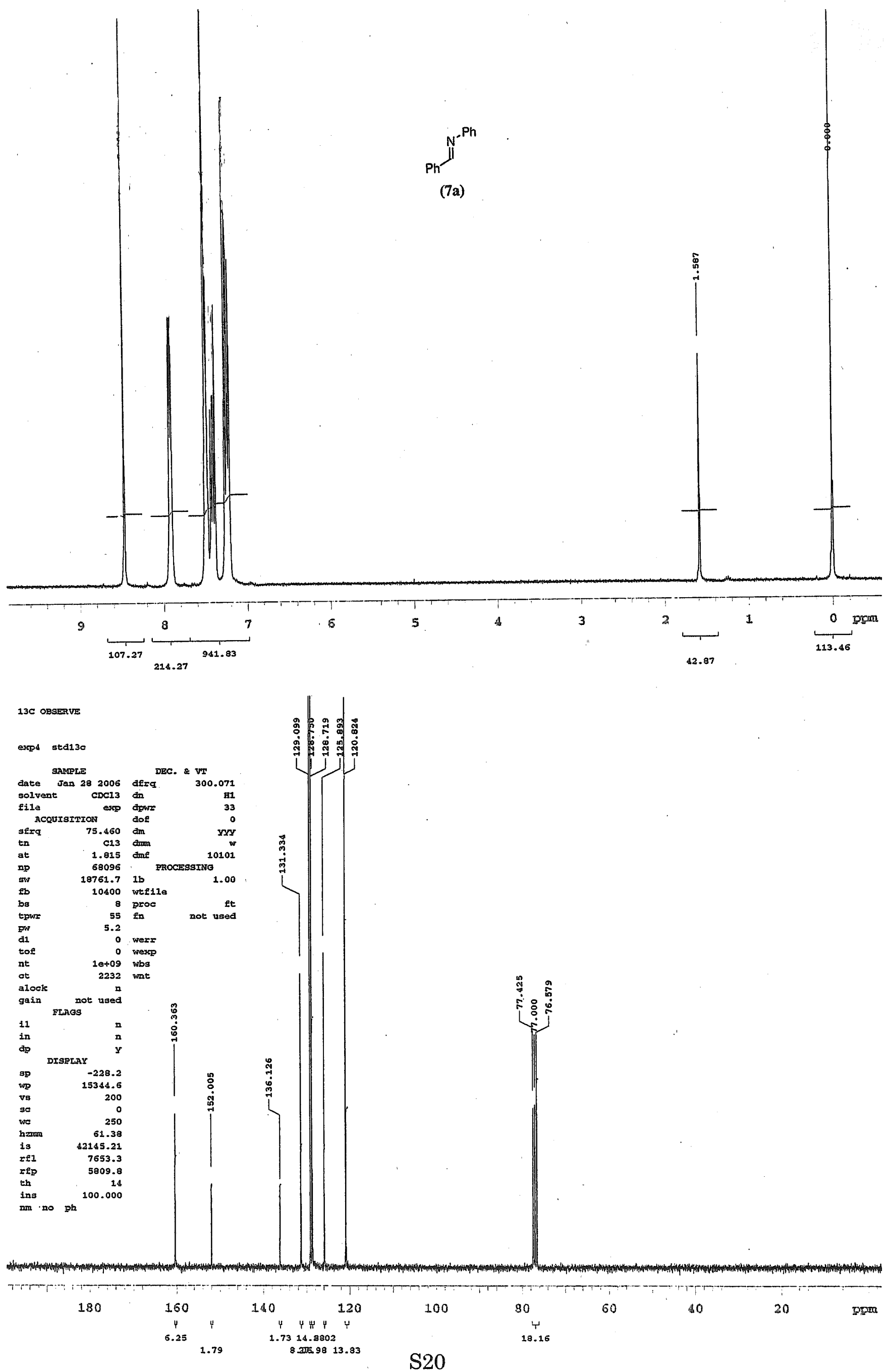


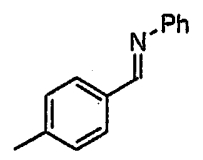

(7b)
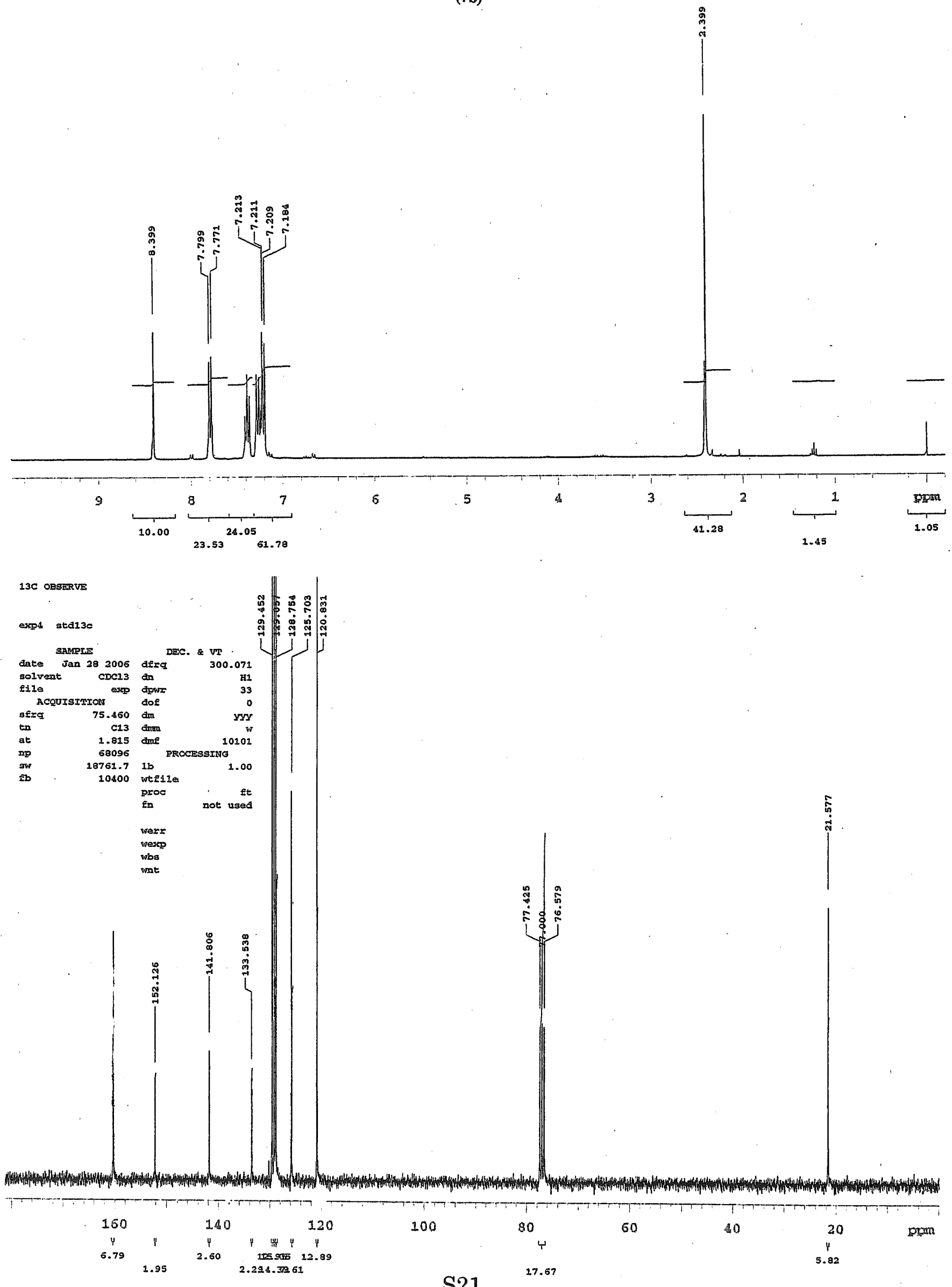
SAMPLEE DEC. \& VT

date Dec 122005 derq 300.071

solvent

file /esport/home/ dpw

cile reporthola dput

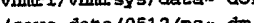

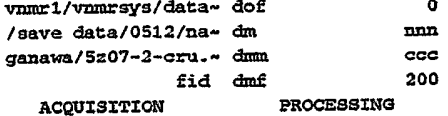

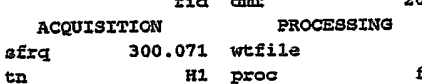

ti 1 HI Proc et

ID $\quad 17984$

to 2600 toer

be 16 wbs

tyowr 50 want

pw 5.2

$\begin{array}{lr}\text { d1 } & 1.000 \\ \text { tof } & 0\end{array}$

$\begin{array}{ll}\text { nt } & 16 \\ \text { et } & 16\end{array}$

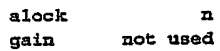

not used
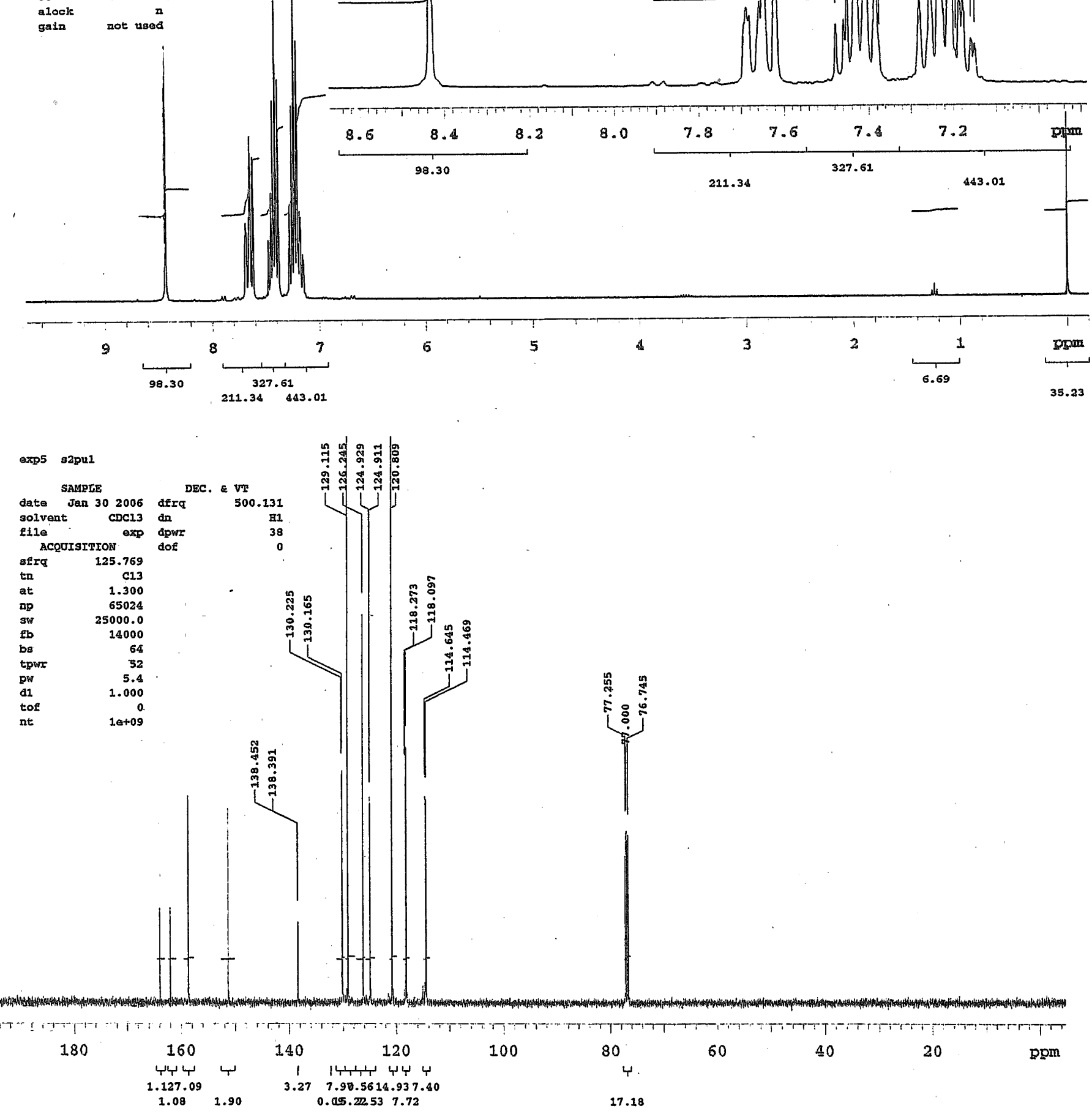

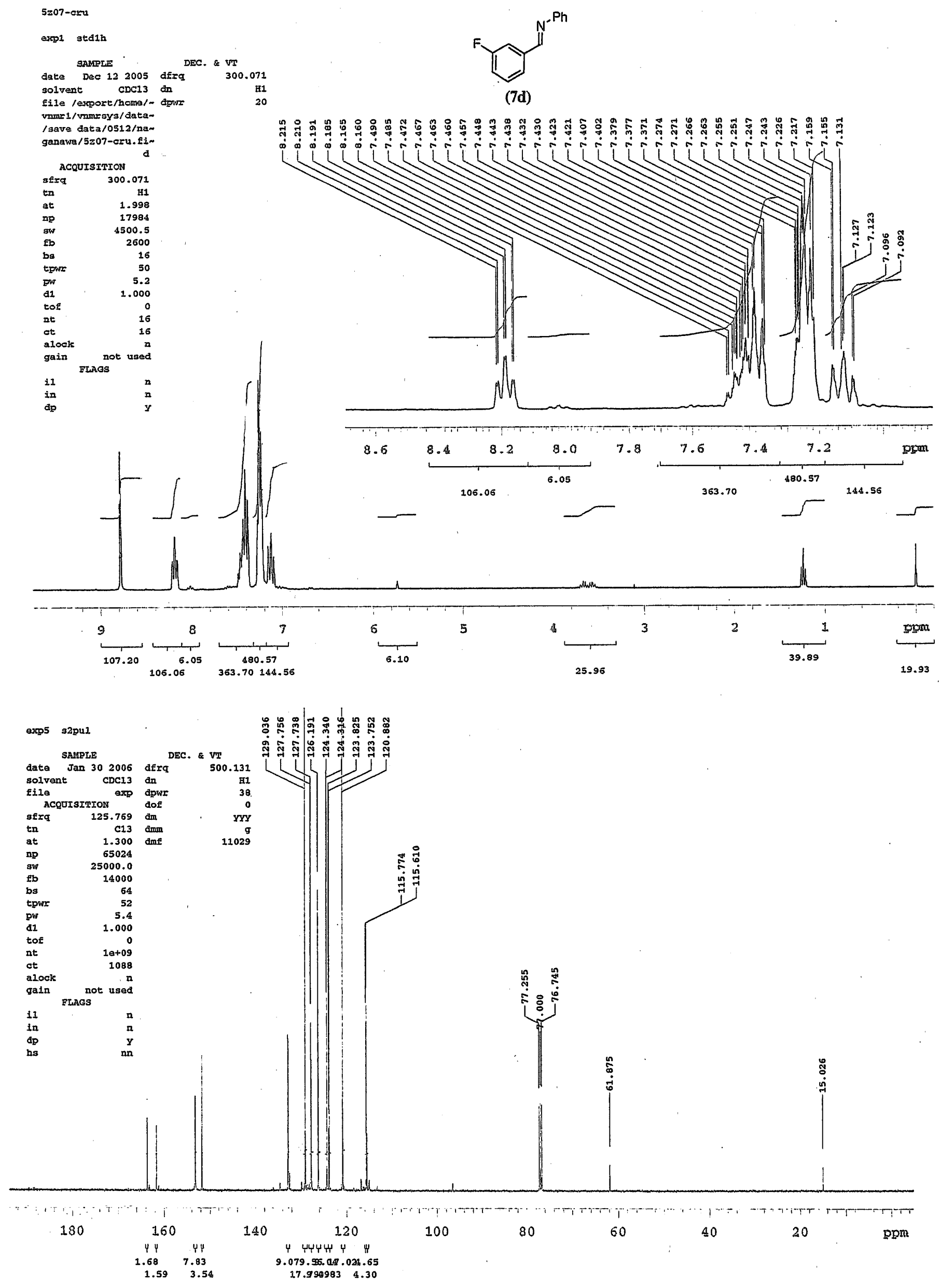


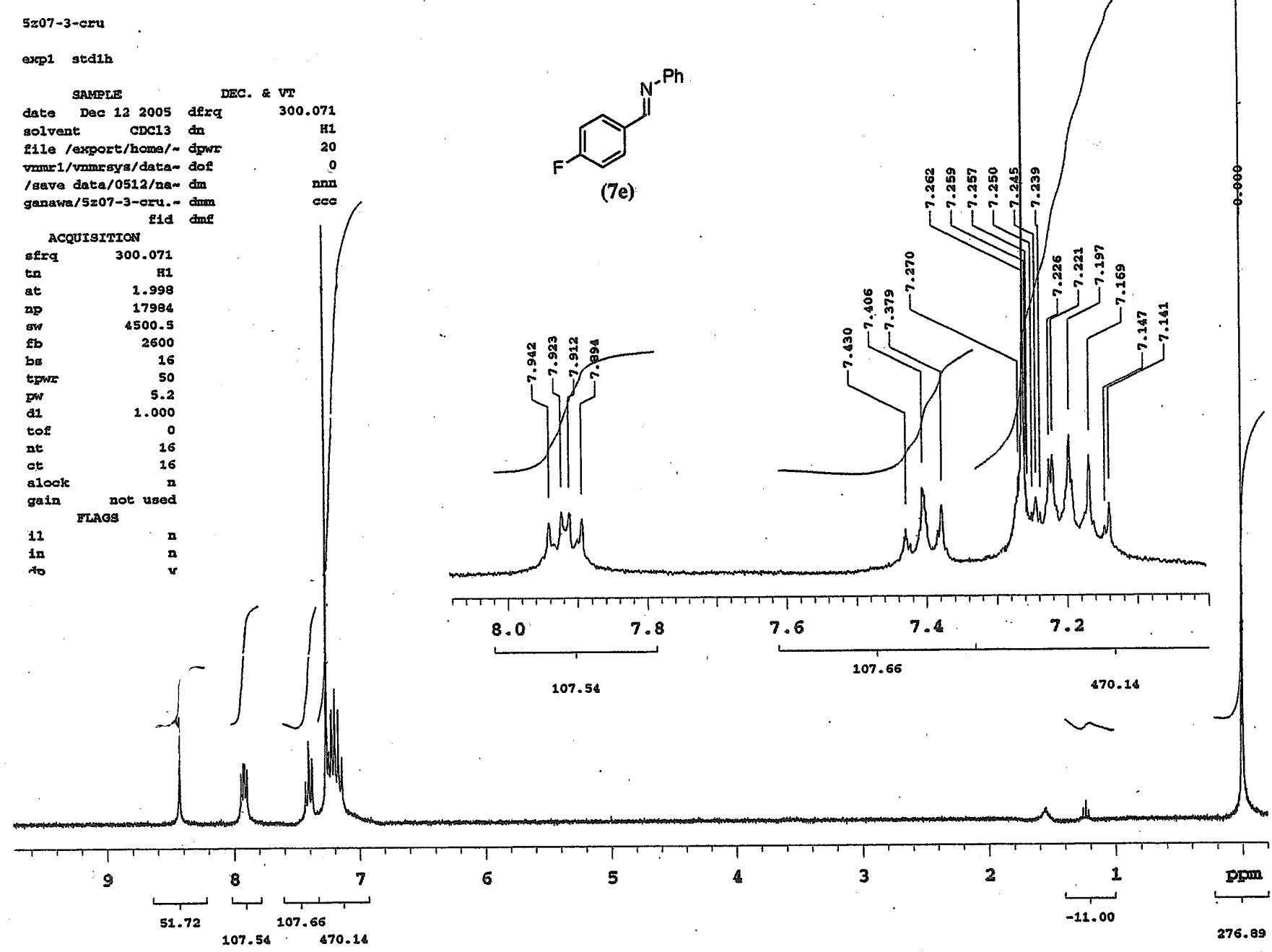

STANDARD CARBON PARAMBTERS

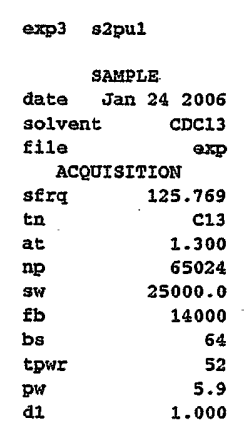

date Jan 24200

ACQUISITION

sfrq $\quad 125.76$

.300

14000

52

.000
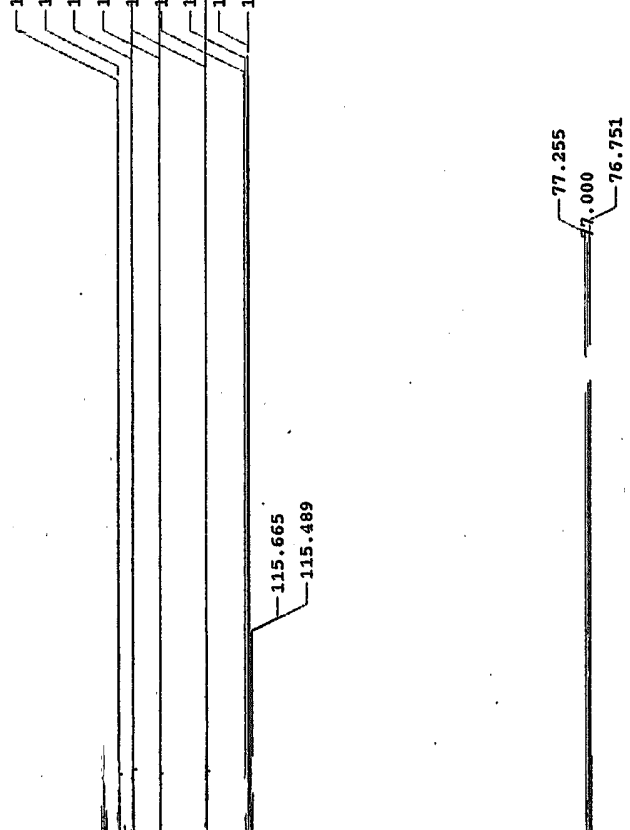

190

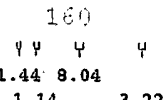

140

220

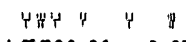

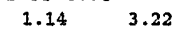

91890516.30 .75

$121: \quad 30$

60

20

Eom 

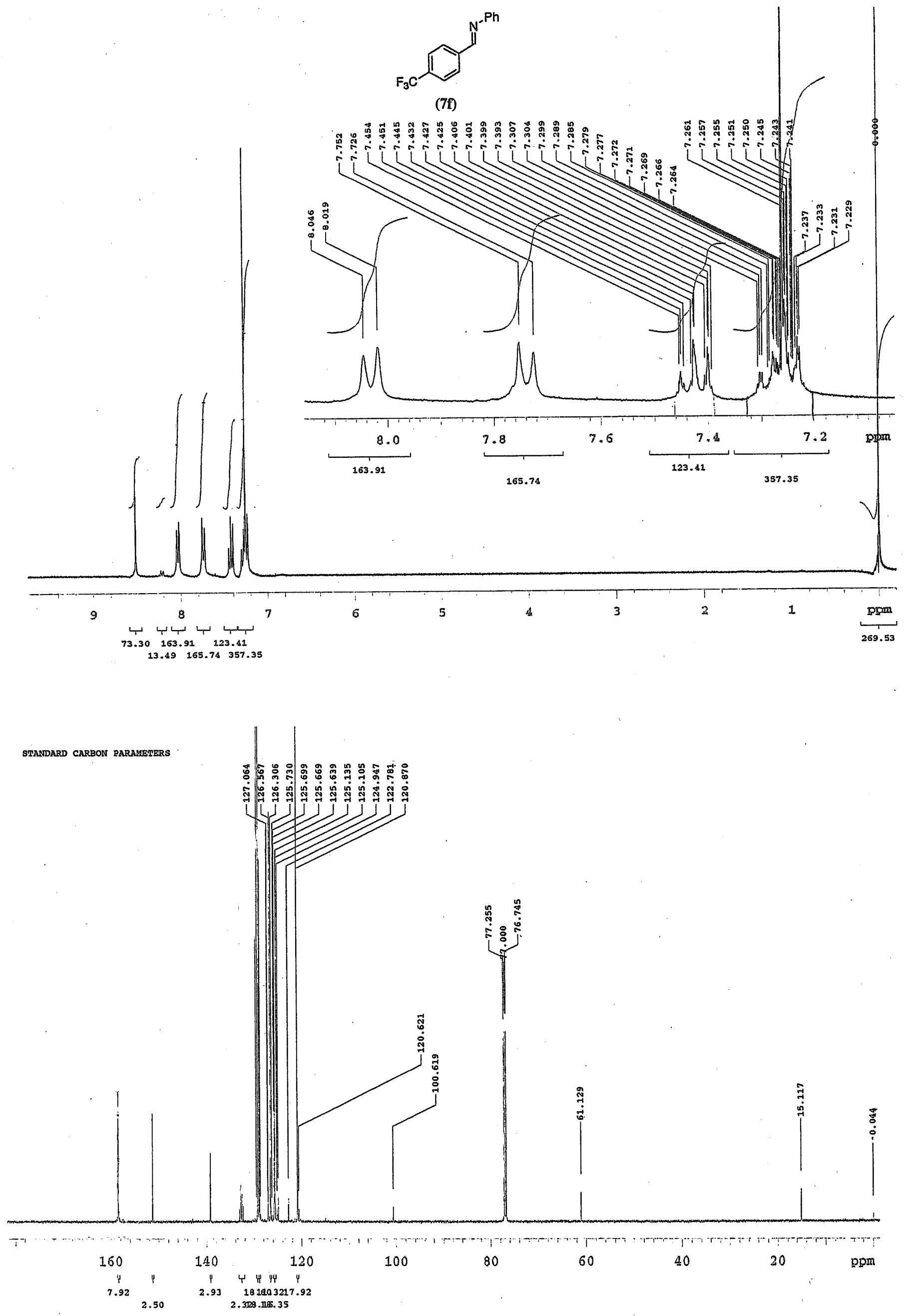
5z06-5-diat

exp2 staih

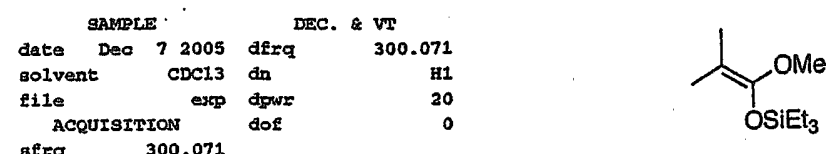

$\begin{array}{lrr}\text { SEEq } & 300.071 & \text { H1 } \\ \text { tn } & 1898\end{array}$

at 1.998

$\begin{array}{lr}\text { sw } & 4500.5 \\ \text { Eb } & 2600 \\ \text { bB } & 16\end{array}$

$\begin{array}{ll}\text { bB } & 16 \\ \text { tpwr } & 50\end{array}$

Dis $\quad \begin{array}{rr}5.2 \\ \text { a1 }\end{array}$

tof $\quad 1.000$

$\begin{array}{lr}\text { nt } & 16 \\ \text { ct } & 16 \\ \text { alook } & n\end{array}$

alook not used

FLAG8

in

$\begin{array}{rr}\text { DISELAYY } & -60.2 \\ \text { WP } & 3000.6\end{array}$

WP $\quad \begin{array}{r}3000.6 \\ \text { Ws }\end{array}$

we 250

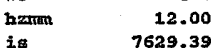

I.1 $\quad 348.0$

$\begin{array}{lr}\text { rfp } & 0 \\ \text { th } & 20 \\ \text { ins } & 100.000\end{array}$

nm sde ph

200

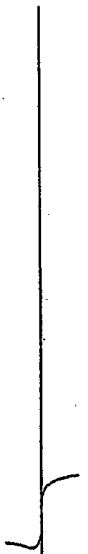

。๊
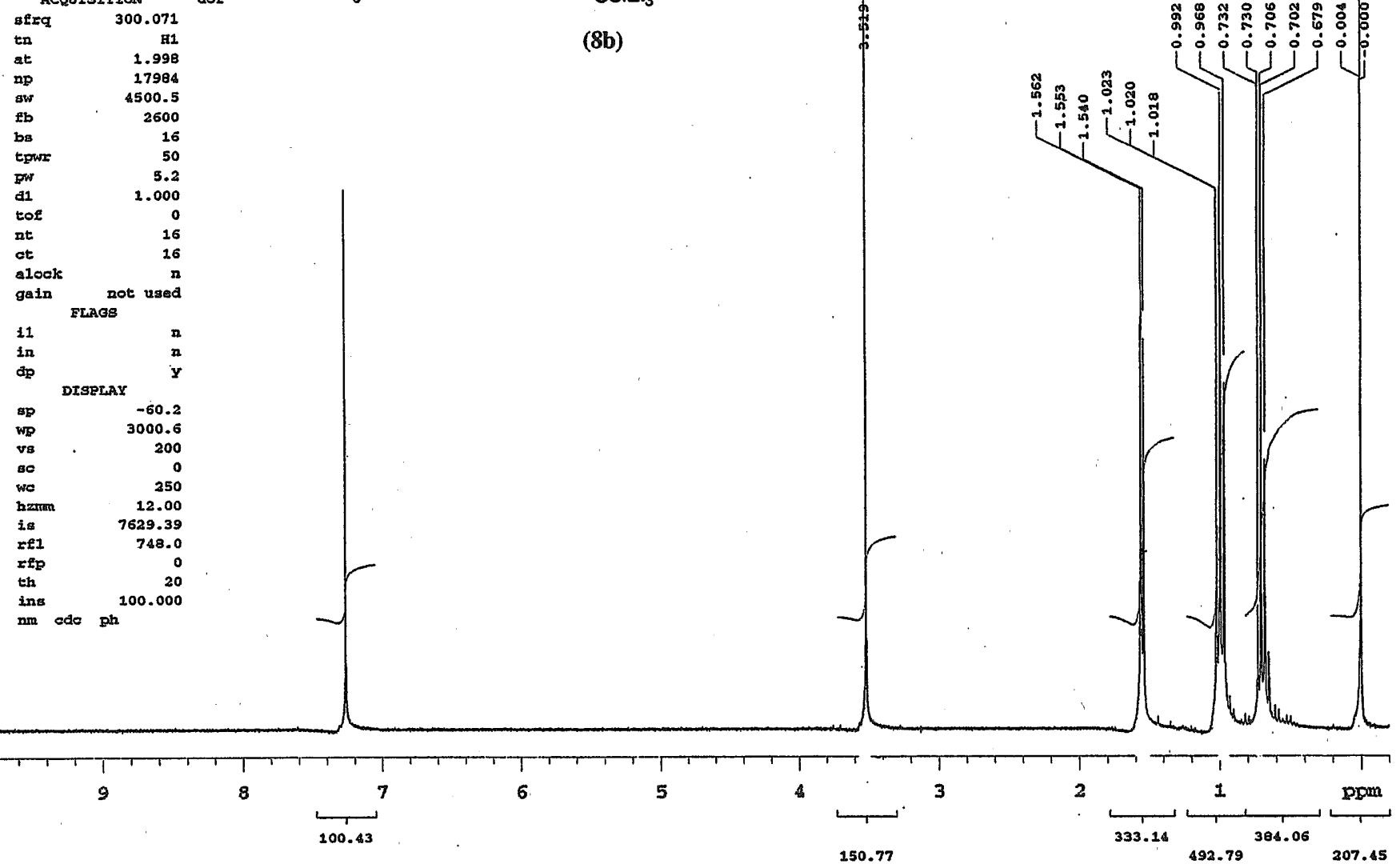

exp2 std13e
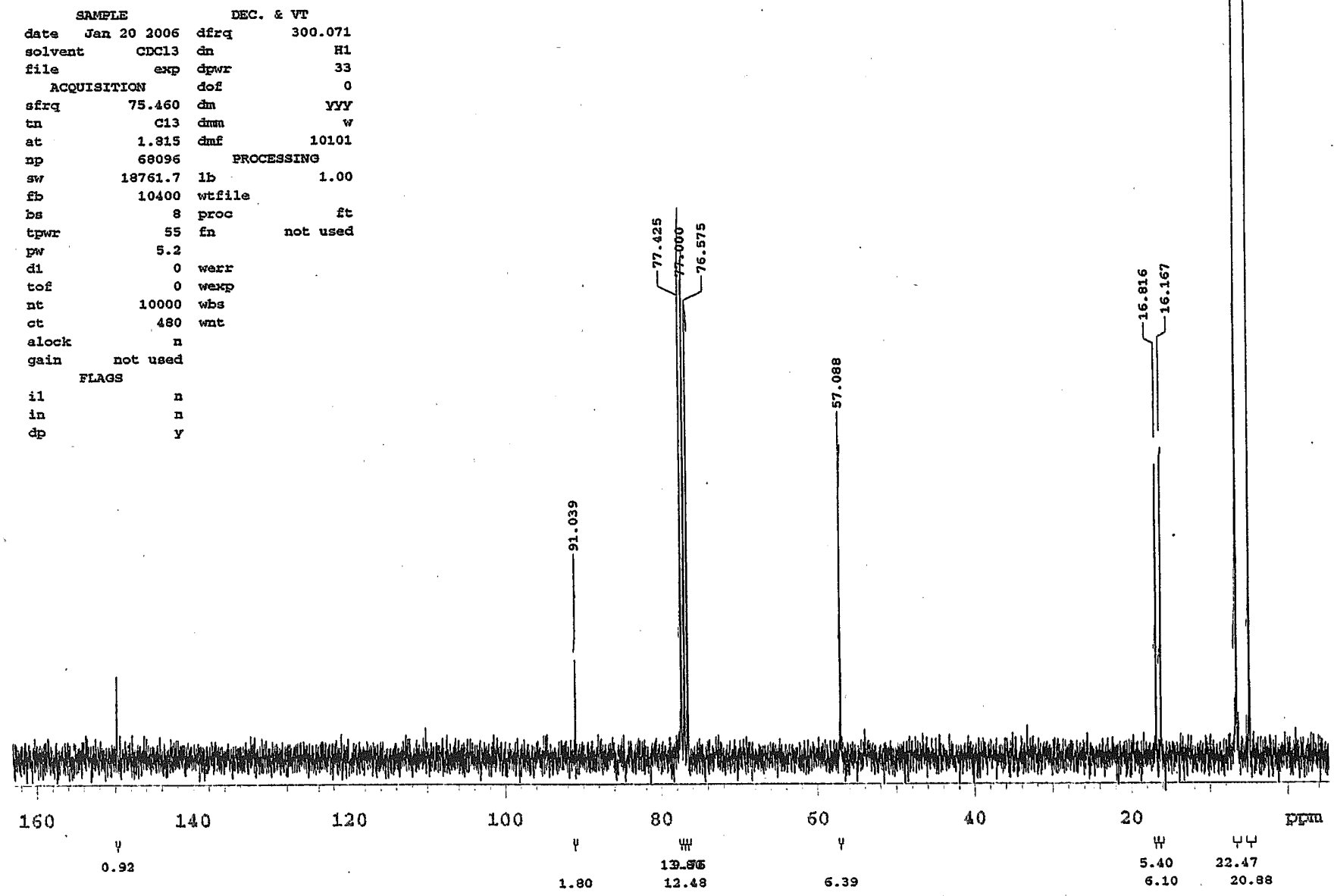
<smiles>COC(=O)C(C)(C)C(Nc1ccccc1)c1ccccc1</smiles>
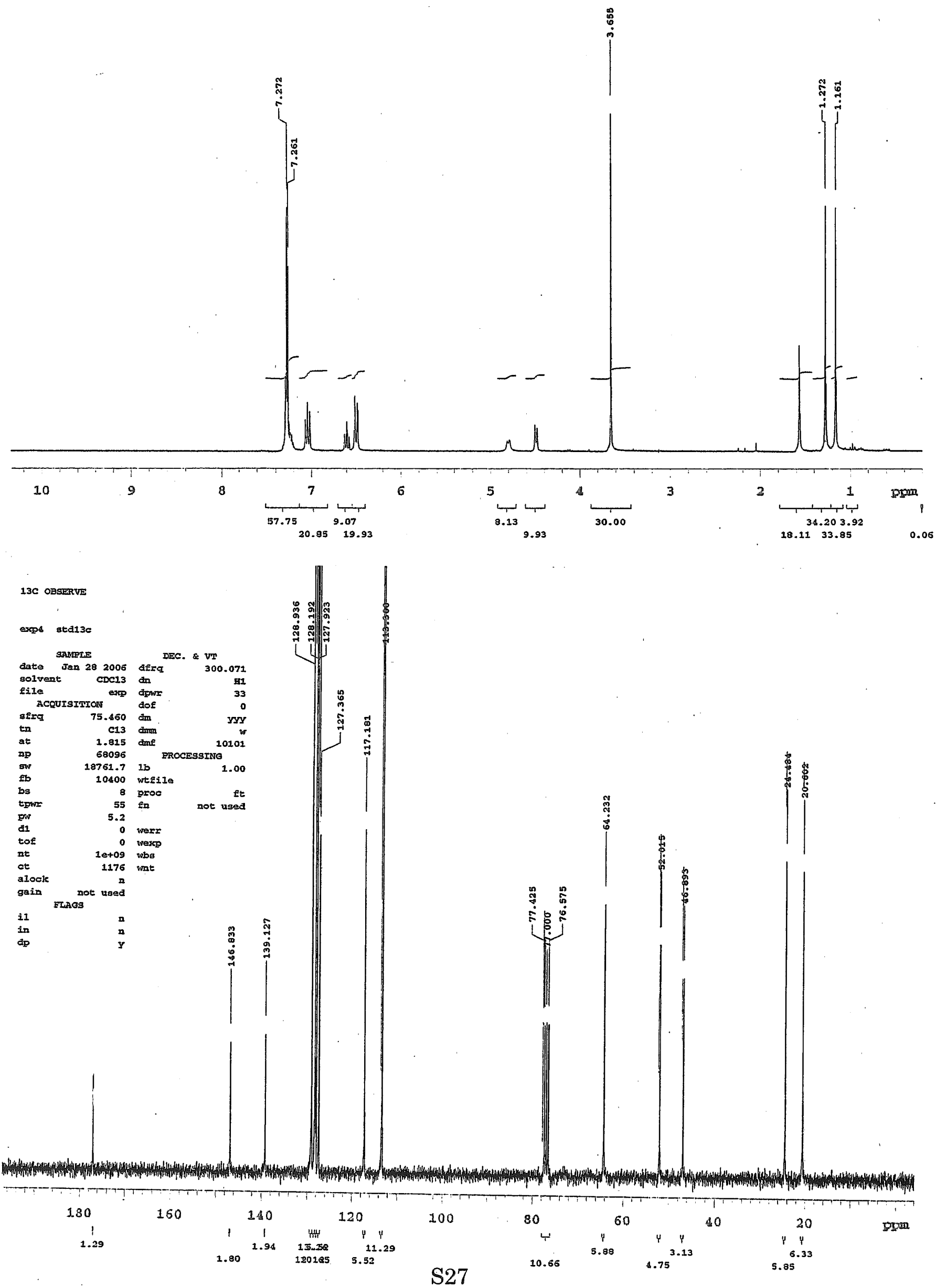

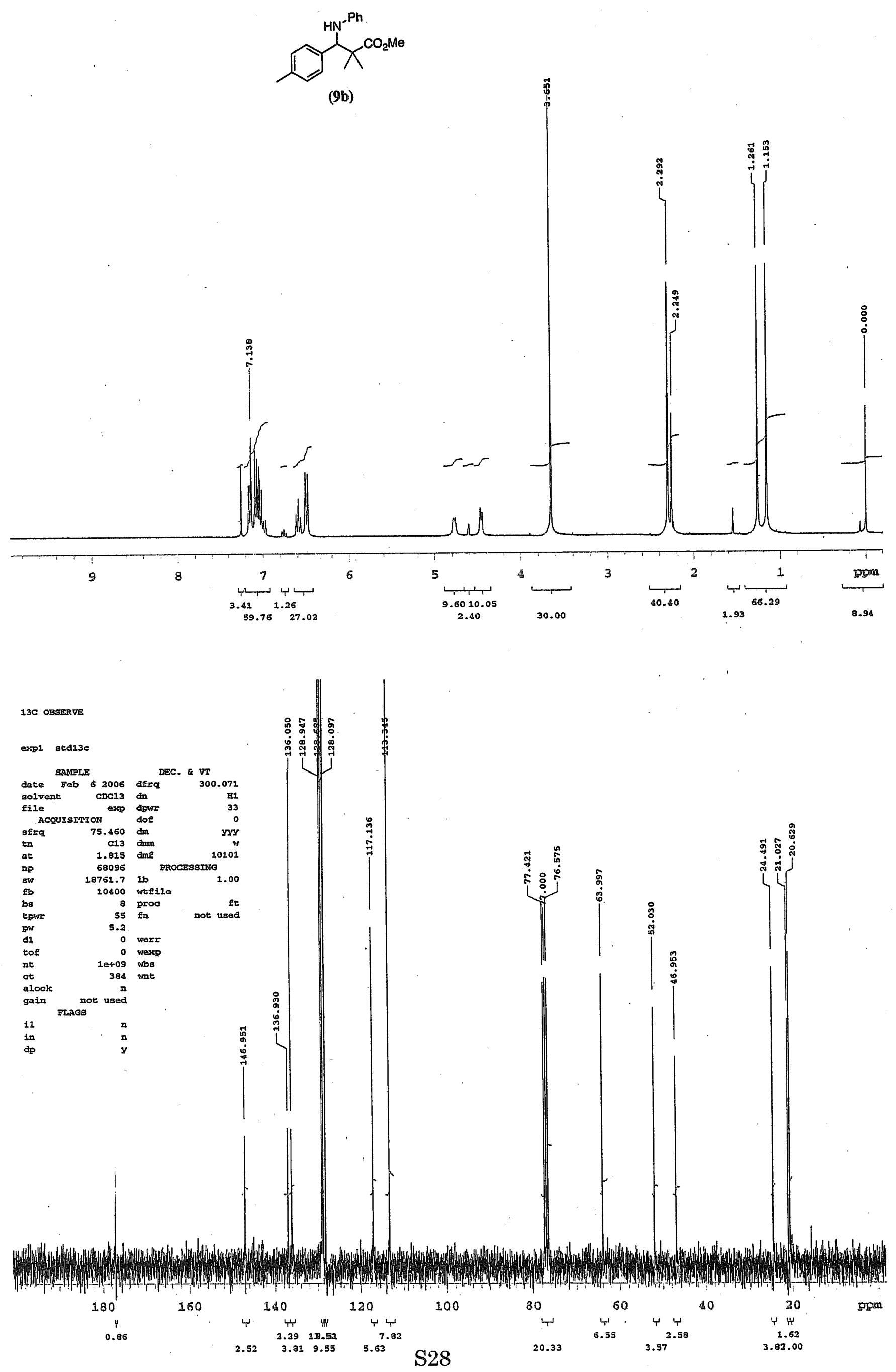


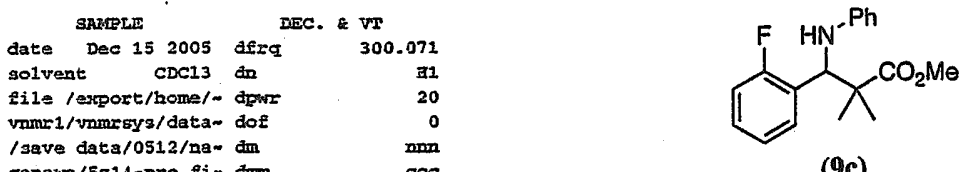

/save data/0512/na- am

(9c)

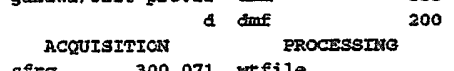

EROCESSIAG
SEFq 300.071 wtfile

$\begin{array}{lrll}\text { girg } & 300.071 & \text { wtfile } \\ \text { tn } & \text { HI } & \text { proe } \\ \text { at } & 1.998 & \text { fn } & \text { not used }\end{array}$

$\begin{array}{lrl}\text { at } & 1.998 & \text { fn } \\ \text { np } & 17984 & \\ \text { sw } & 4500.5 & \text { warx }\end{array}$

sw $\quad 4500.5$ werx

$\begin{array}{lll}\text { be } & 16 & \text { wbe } \\ \text { tpwI } & 50 & \text { int }\end{array}$

$\begin{array}{lr}\text { tpwI } & 50 \\ \text { pw } & 5.2 \\ \text { di } & 1.000\end{array}$

$\begin{array}{lr}\text { di } & 1.000 \\ \text { tot } & 0 \\ \text { at } & 16\end{array}$

$\begin{array}{lrl}\text { tot } & 16 & \text { st } \\ \text { at } & 16 & \text { s. }\end{array}$

gain not used

$\begin{array}{ll}\text { i1 } & \text { n } \\ \text { in } & \text { n } \\ \text { dp } & y\end{array}$

dPSPLAY

$\begin{array}{ll}\text { ap } & -105.2 \\ \text { WP } & 3151.7\end{array}$

$\begin{array}{lr}\text { wP } & 3151.7 \\ \text { vs } & 200 \\ \text { sc } & 0 \\ \text { we } & 250\end{array}$

$\begin{array}{lr}\text { se } & 250 \\ \text { we } & 12.61\end{array}$

$\begin{array}{lr}\text { is } & 12875.00 \\ \text { r.1 } & 749.7\end{array}$

$\begin{array}{lr}\text { th } & 0 \\ \text { th } & 11\end{array}$

ins ede ph 100.000
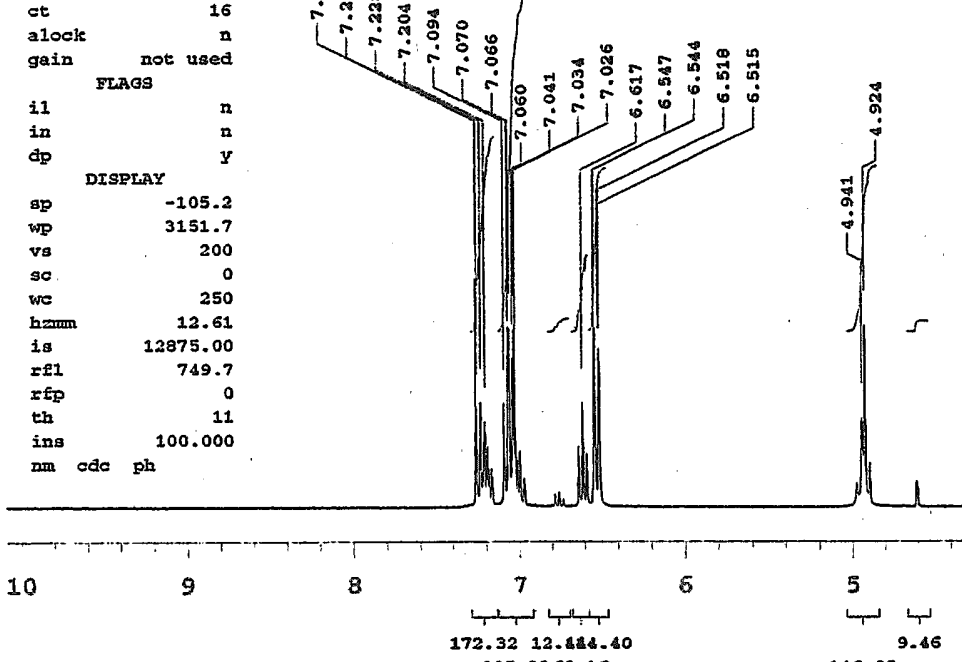

295.2268 .16

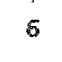

6

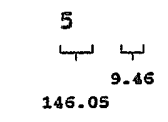

4 216.57
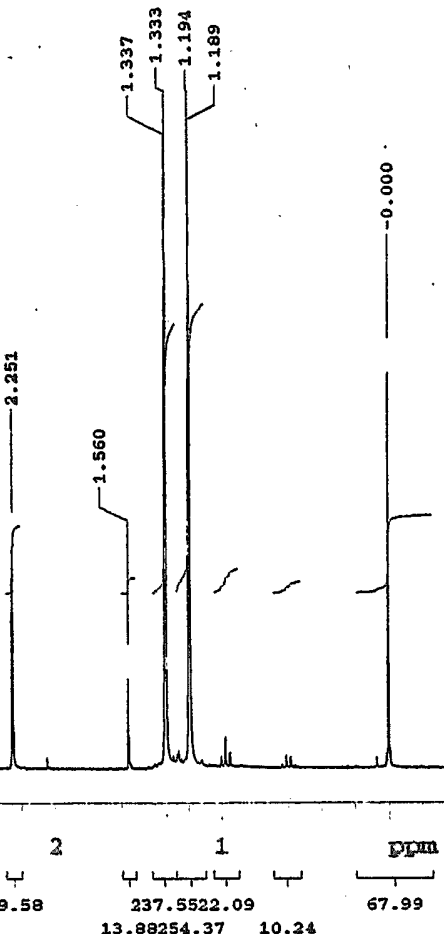

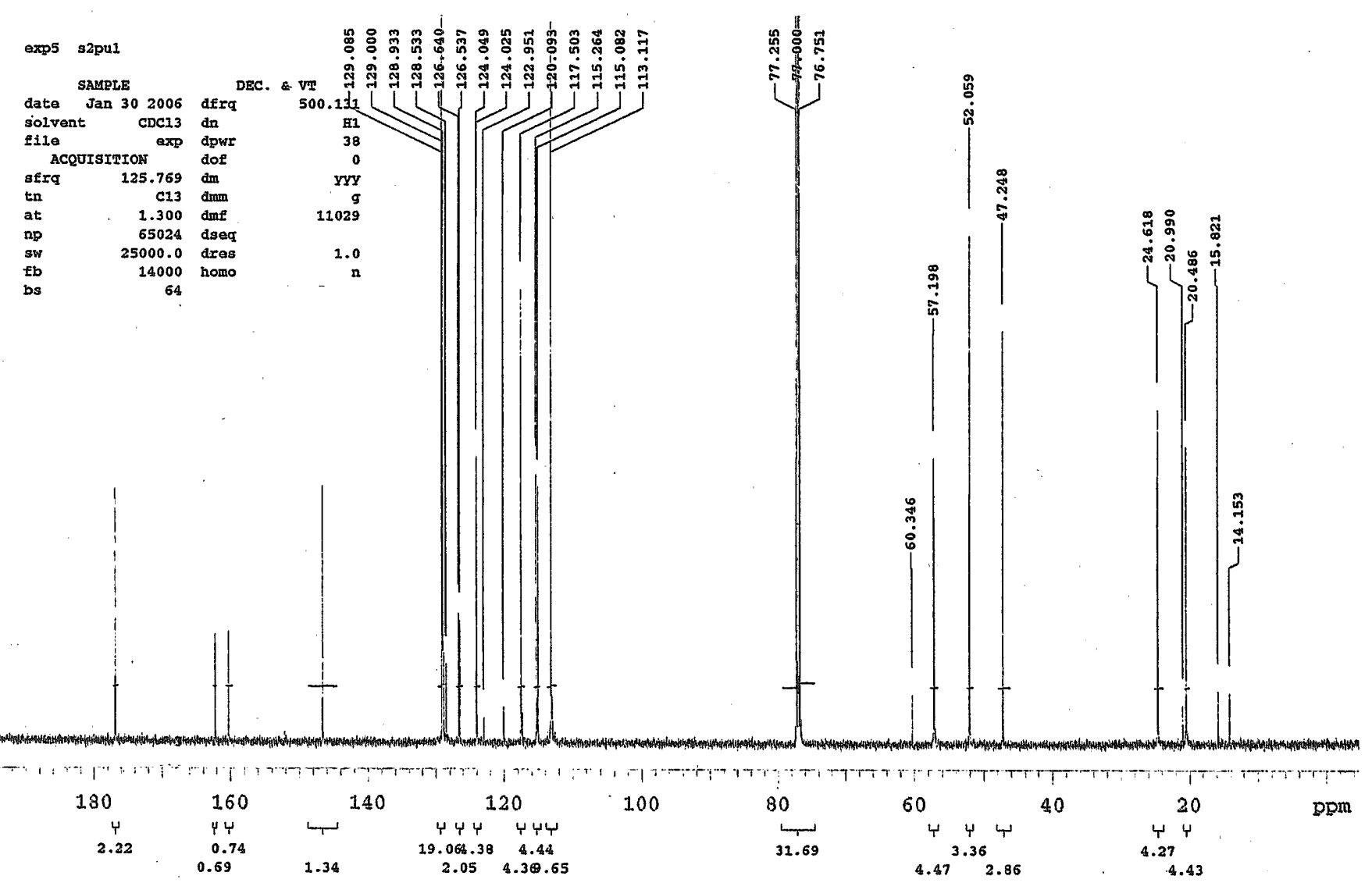


$\begin{array}{lll}\text { SARTLE } & \text { DEC. \& VI } \\ \text { date DeC } 152005 \text { dfrg } & 300.071\end{array}$

$\begin{array}{lr}\text { solvent CDCl3 di } & \text { H1 } \\ \text { iile /esport/home/ dews } & 20 \\ \text { vnmr1/vamssys/datam dof } & 0\end{array}$

/save data/0512/aar dim
ganawa/5z14-2-pro.- drm

ACQUISITION Eid dimé EROCEssINo 200

sfrq 300.071 tefile

$\begin{array}{lrlr}\text { tn } & \text { H1 } & \text { proc } \\ \text { at } & 1.998 & \text { fn } \\ \text { nP } & 17984 & & \text { not used }\end{array}$

SP $\quad 17984$. 4500.5 wers

$\begin{array}{rrr}\text { tb } & 2600 & \text { wexp } \\ \text { bs } & 16 & \text { wbs }\end{array}$

$\begin{array}{lll}\text { bs } & 16 & \text { wbs } \\ \text { tpors } & 50 & \text { wat }\end{array}$

$\begin{array}{lr}\text { tpwr } & 50 \\ \text { pw } & 5.2 \\ \text { d1 } & 1.000 \\ \text { tof } & 0\end{array}$

$\begin{array}{lr}\text { tof } & 0 \\ \text { nt } & 16 \\ \text { et } & 16 \\ \text { alock } & \text { In } \\ \text { gain } & \text { not used }\end{array}$

in $n$

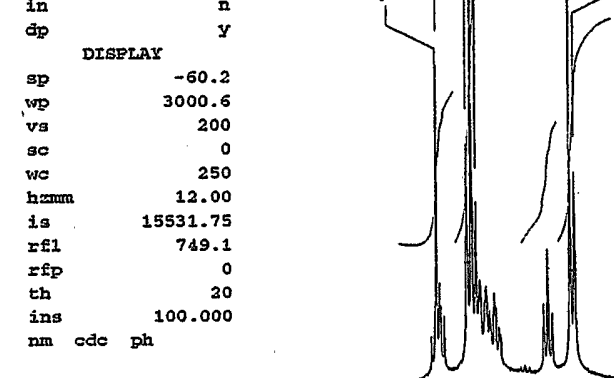

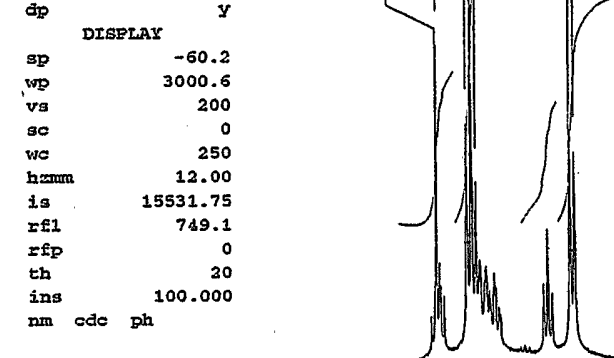

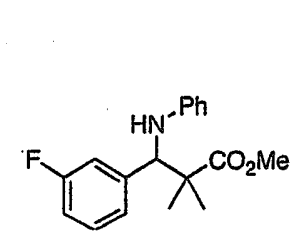

(9d)

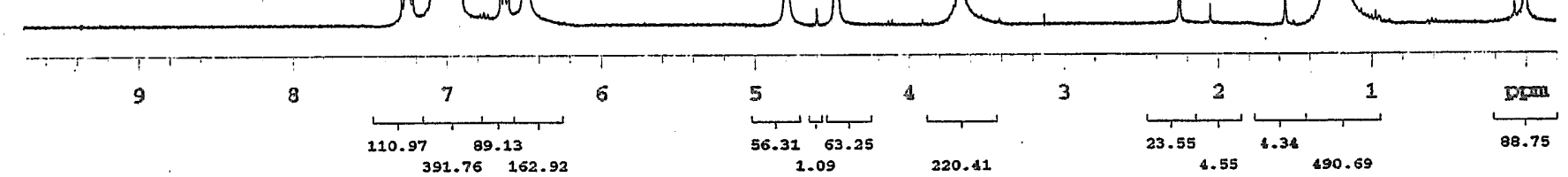

STAMDARD CARBON PARAMETERS

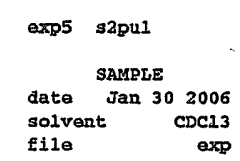

solvent
file

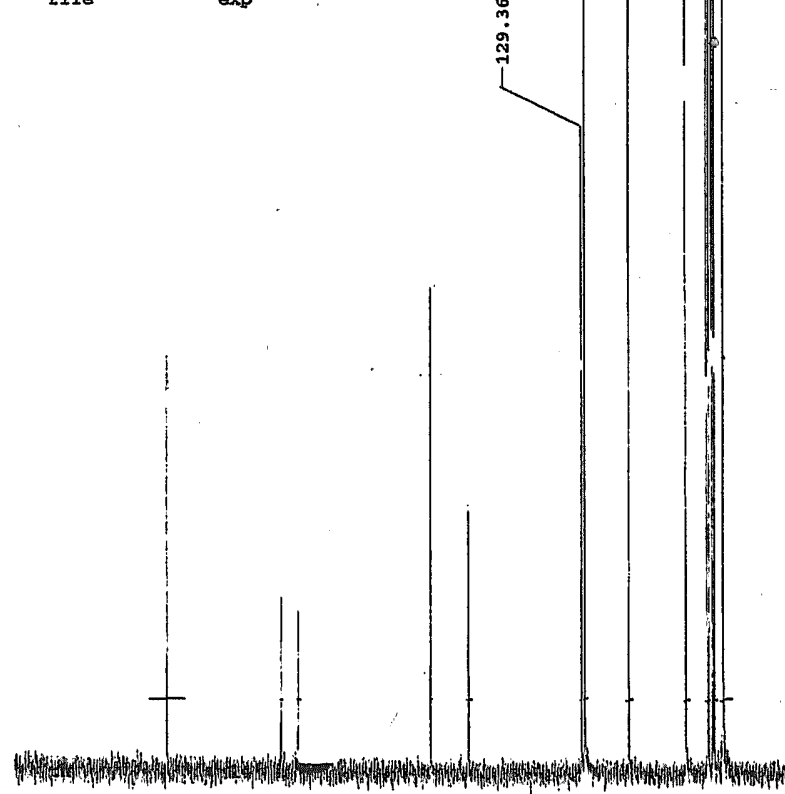

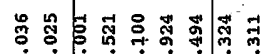
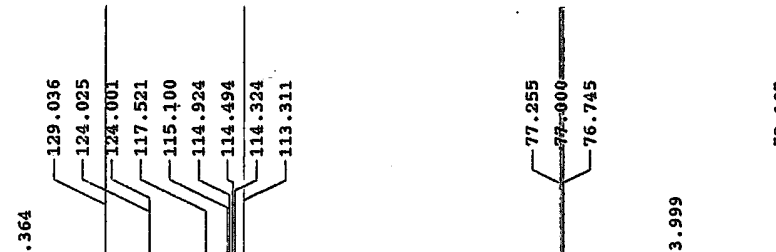

ذิ

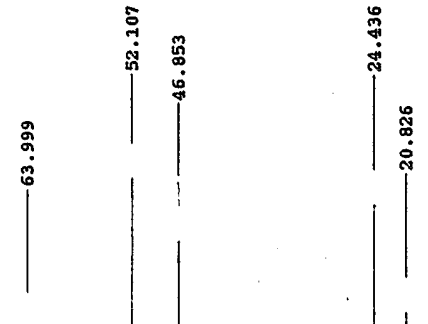

$\stackrel{g}{\circ}$
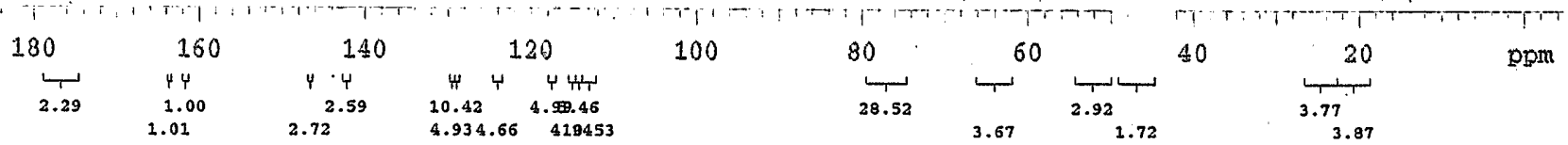


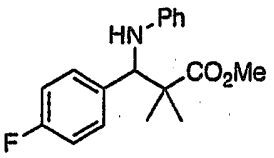

(9e)
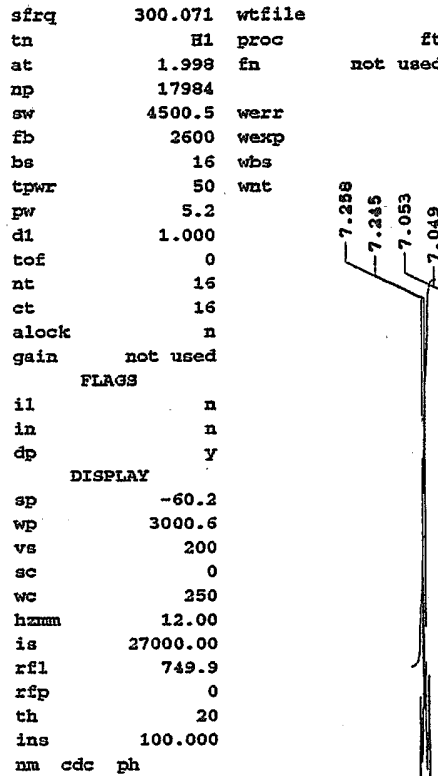

not need
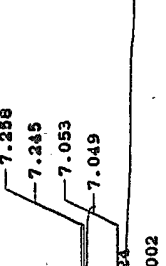

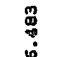
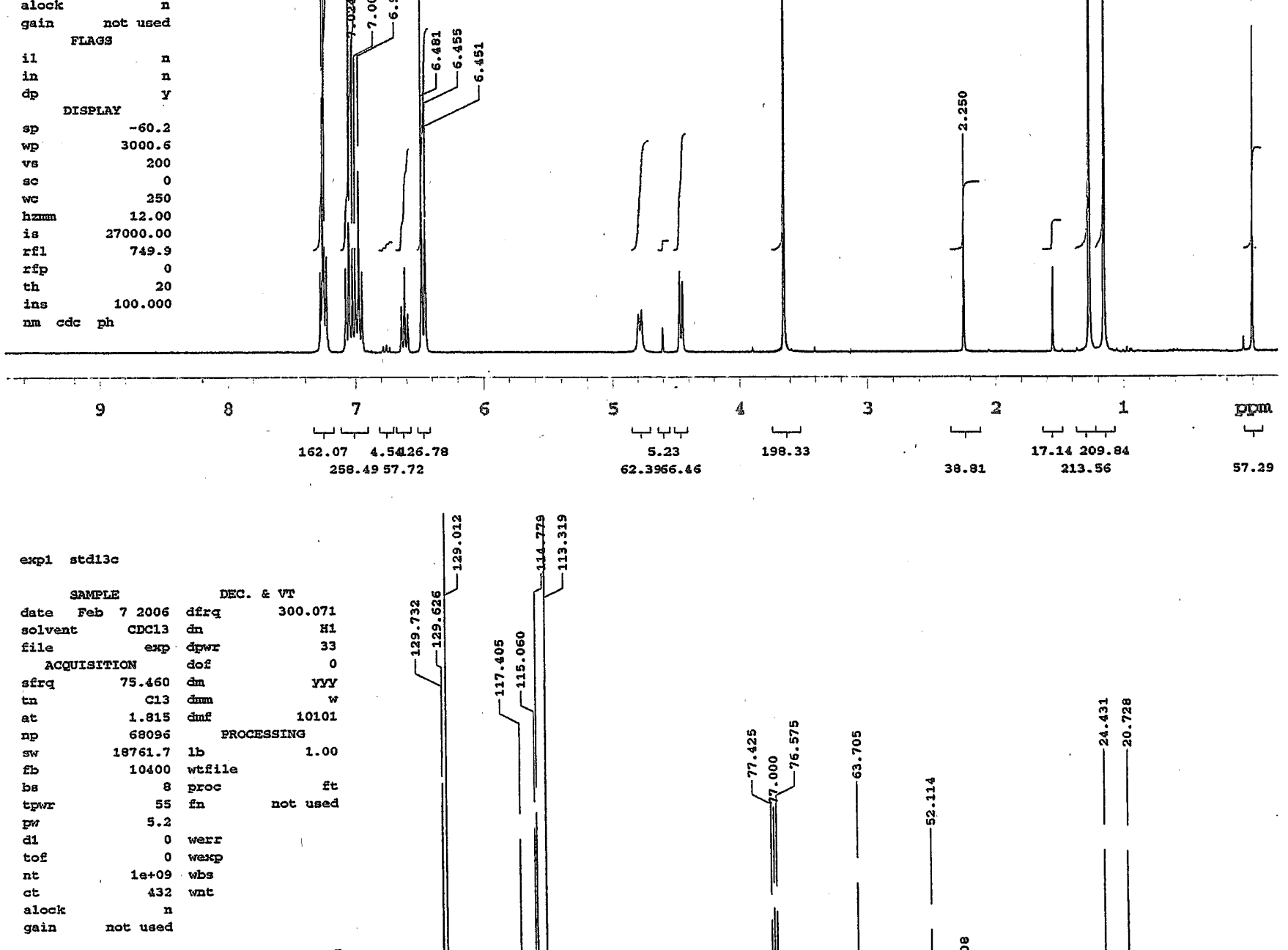

\section{隶}
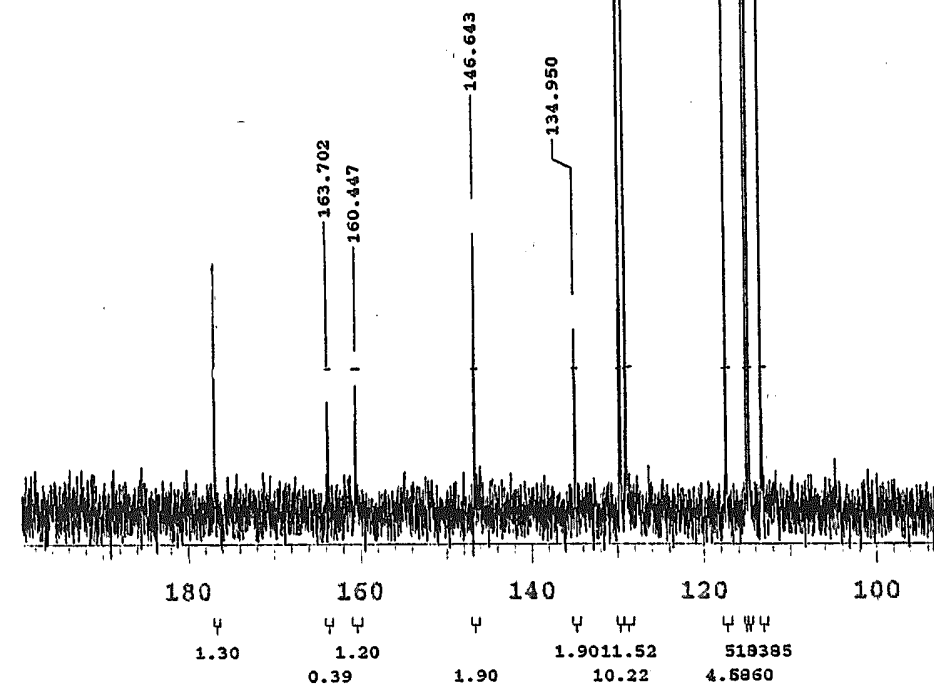
Eulse Sequence: 32pu1

Solvent: $\operatorname{CDC13}$.

Ambient temperature

File:

GEXINI-300BB "varian"

Re1ax. delay $1.000 \mathrm{sec}$

Pulse 45.0 degrees

ACq. time $1.998 \mathrm{sec}$

wiath $4500.5 \mathrm{~Hz}$

16 repetitions

DATA PROCESSTNO

FT size 32768

Total time 0 min, 49 sec

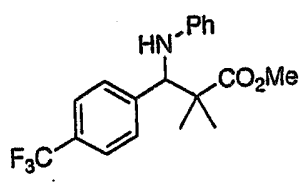

(9f)
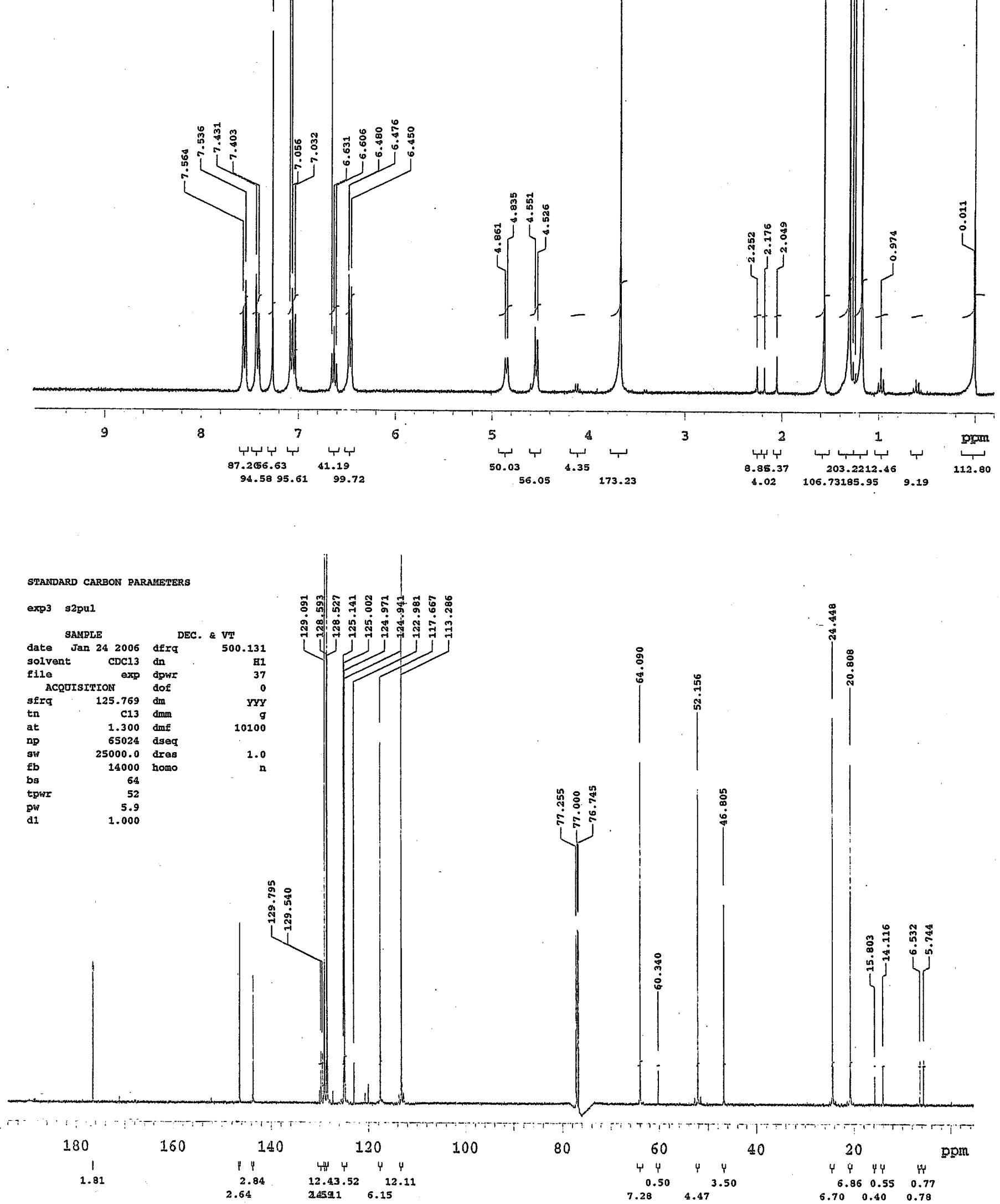

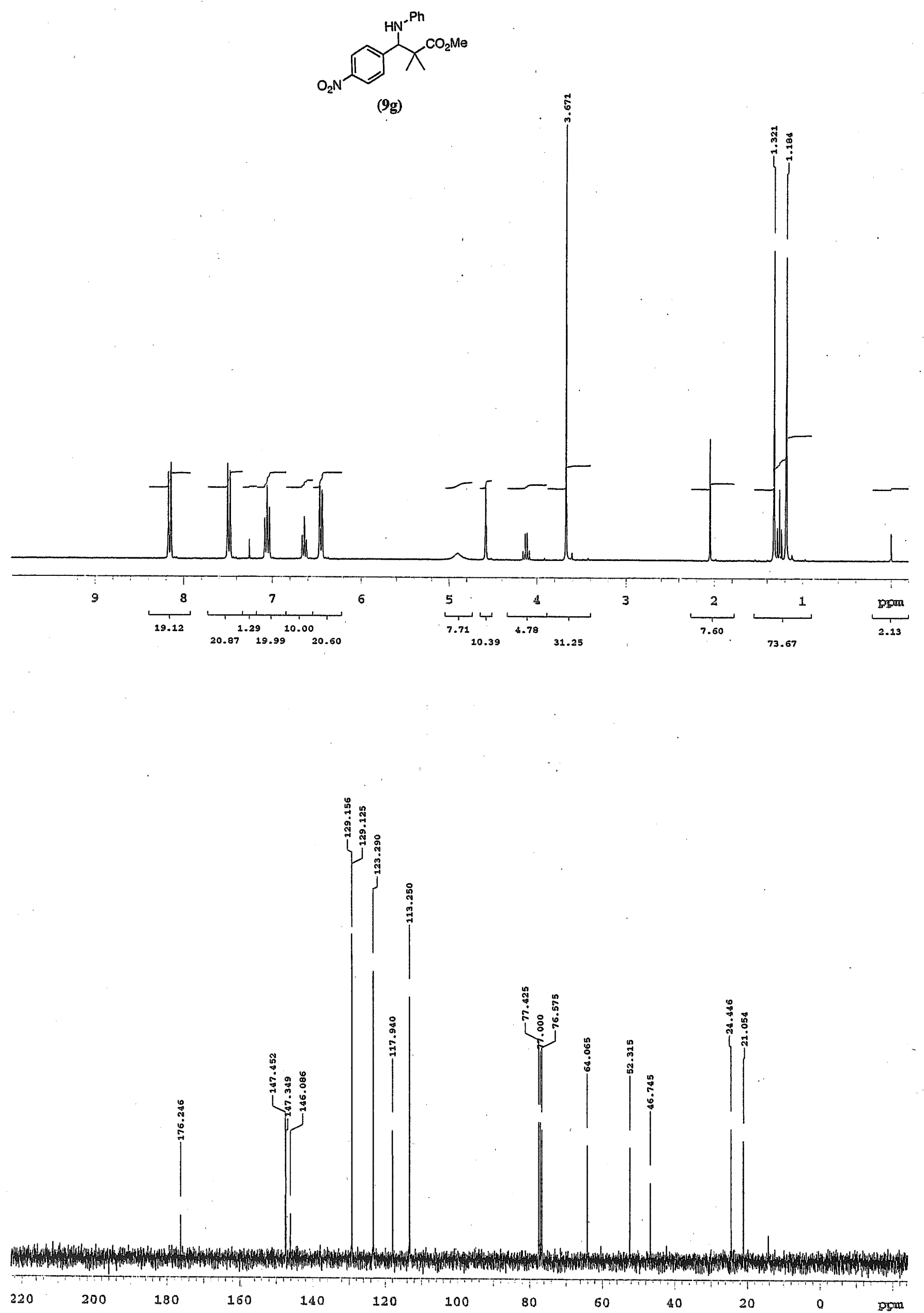
<smiles>C(=N/c1ccccc1)\c1ccccc1</smiles>

(10a)

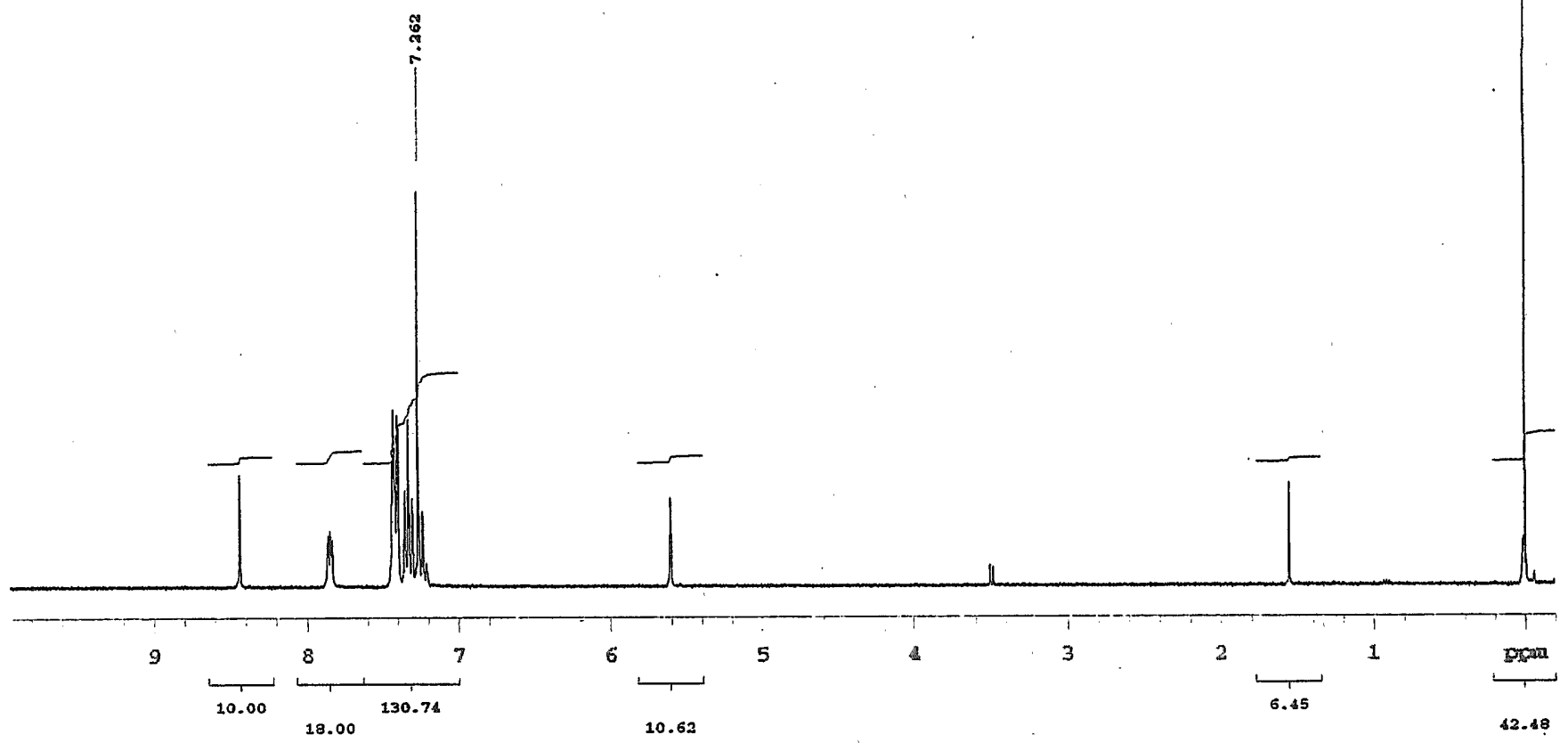

13C OBSERVE

expes sta13c

SARTLE DEC. \& VTI

date Jen 262006 derq 300.071

Bolvent $\mathrm{CDCl}_{3}$ an

lile /esport/home/ dpwr 33

/save data/0601/nan dof

ganawa/diphimize-c- drm 10101

ACQUISITION EROCESSINO

$\begin{array}{lrlr}\text { sirq } & 75.460 & \text { lb } & 1.00 \\ \text { tz } & c 13 & \text { wtfile } & \\ \text { at } & 1.815 & \text { proc } & \text { et } \\ \text { ng } & 68096 & \text { not used }\end{array}$

nP $\quad 68096$

ib $\quad \begin{array}{rll}\text { aw } & 18761.7 & \\ \text { bb } & 10400 \text { werr }\end{array}$

bo 8 wexp

$\begin{array}{rrr}55 & \text { whos } \\ \text { pow } & 5.2 & \text { wat }\end{array}$

div $\quad 5.2$

$\begin{array}{lr}\text { tof } & 0 \\ \text { nt } & 1 e+09 \\ \text { et } & 520\end{array}$

$\begin{array}{lr}\text { et } & 520 \\ \text { alook } & n\end{array}$

gain not used

ELAes

n

DISELAY

-136.0
15.23 .5

wP 15.22 .5

$\begin{array}{lr}\text { vs } & 200 \\ \text { sc } & 0\end{array}$

$\begin{array}{lr}\text { we } & 250 \\ & 61.69\end{array}$

$\begin{array}{lr}\text { batrue } & 61.69 \\ \text { is } & 73715.49\end{array}$

$\begin{array}{ll}\text { rfI } & 7654.1 \\ \text { IfP } & 5809.8\end{array}$

th

100.000

ns $\mathrm{no}$. $\mathrm{ph}$
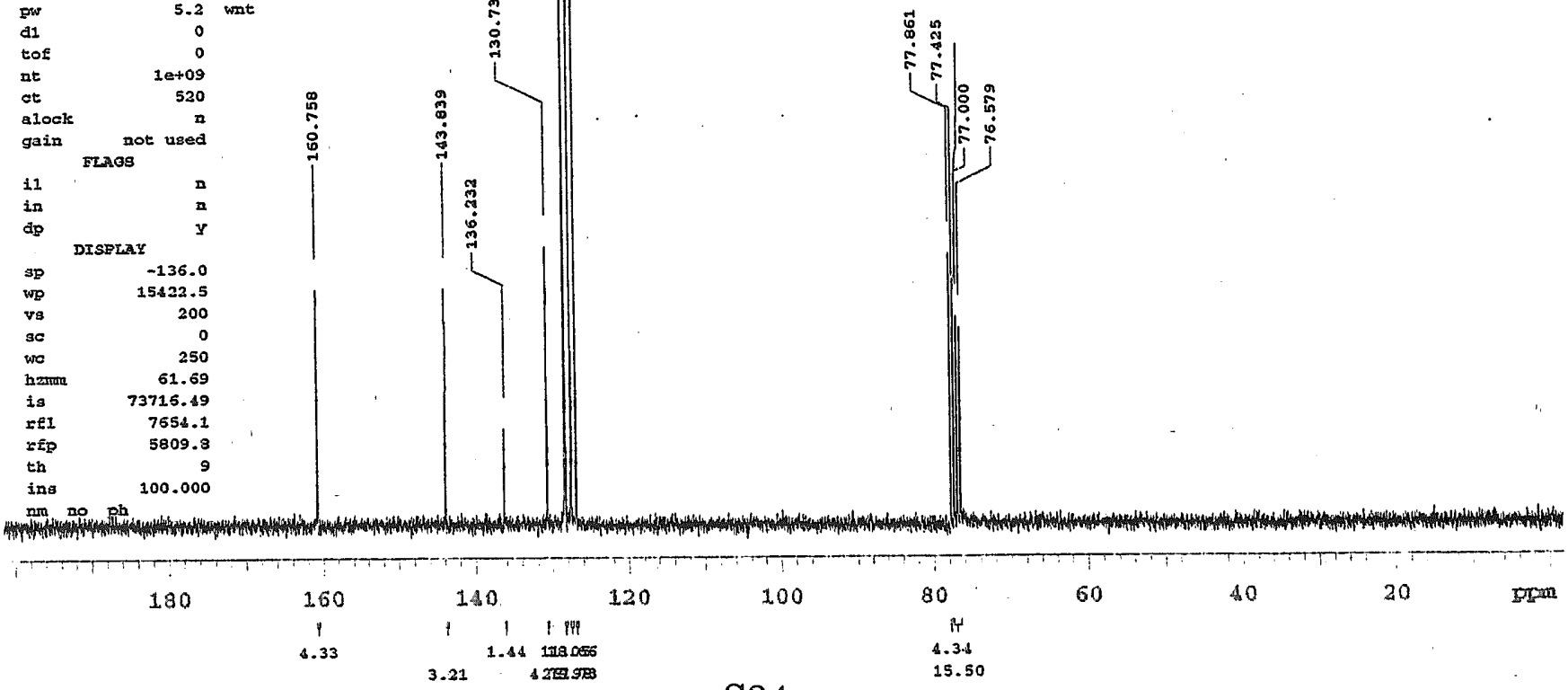

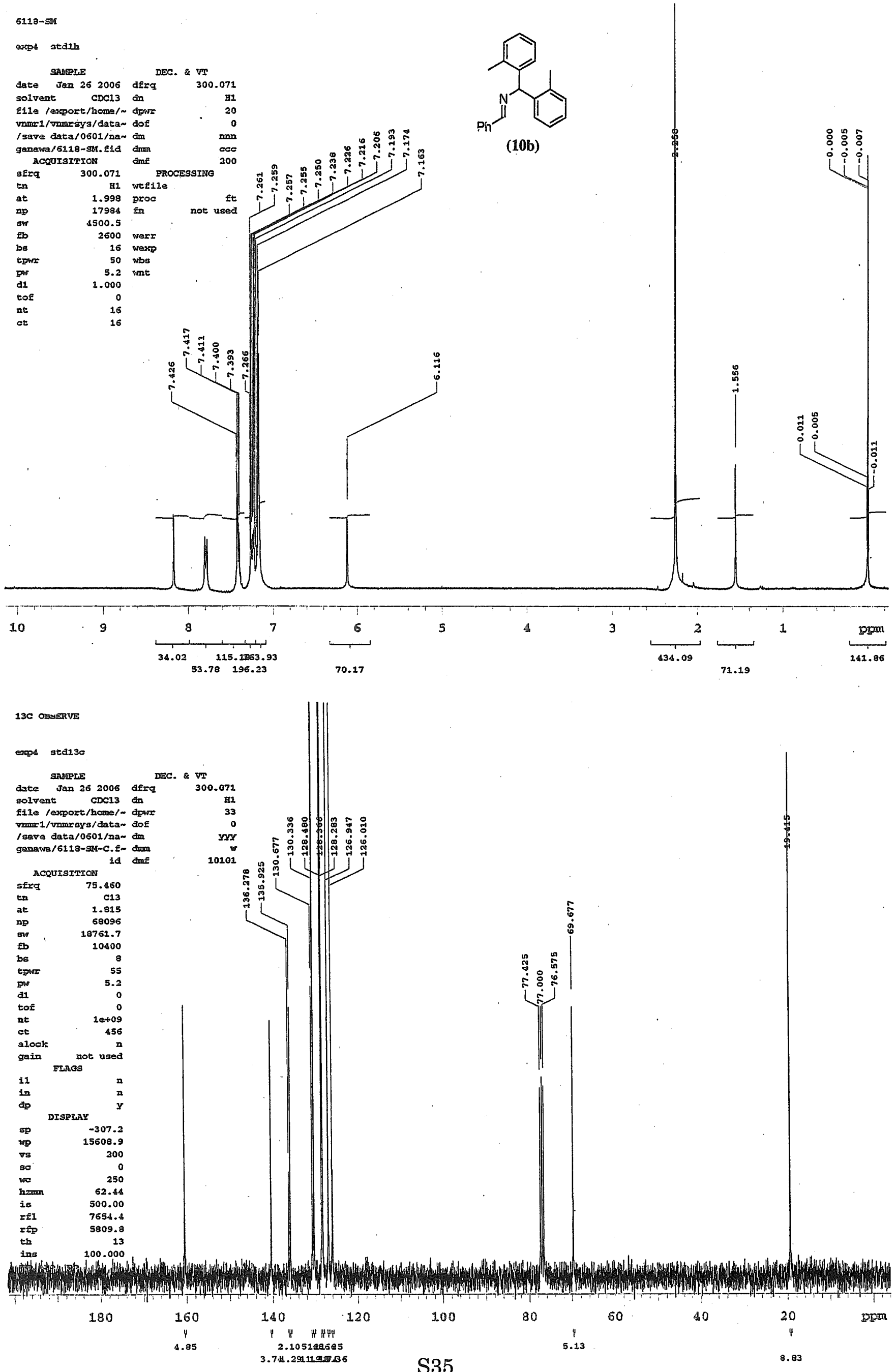
5606-imine-cogein1

erpas sedich

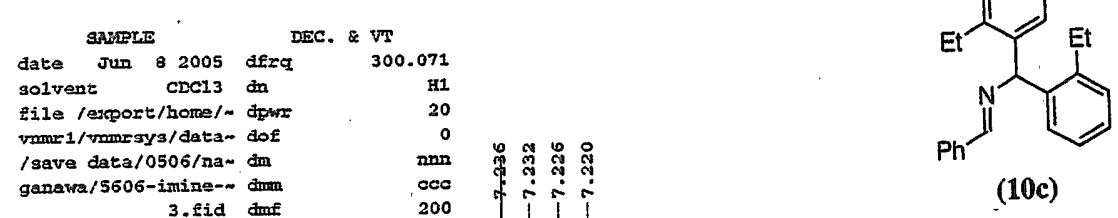

ACRUISITION 3. RROESSINO 200

sfra 300.071 wtile of

$\begin{array}{lrl}\text { tn } & \text { H1 } & \text { groc } \\ \text { at } & 1.998 & \text { not used }\end{array}$

$\begin{array}{lr}\text { ap } & 17984 \\ \text { sw } & 4500.5\end{array}$

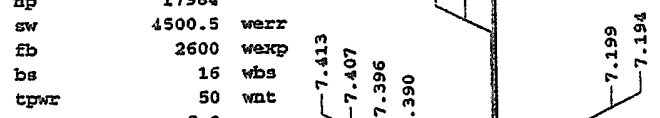

di

1.000

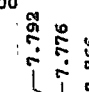

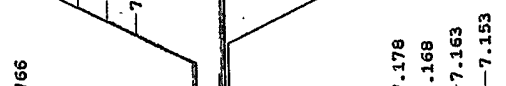

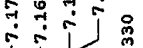
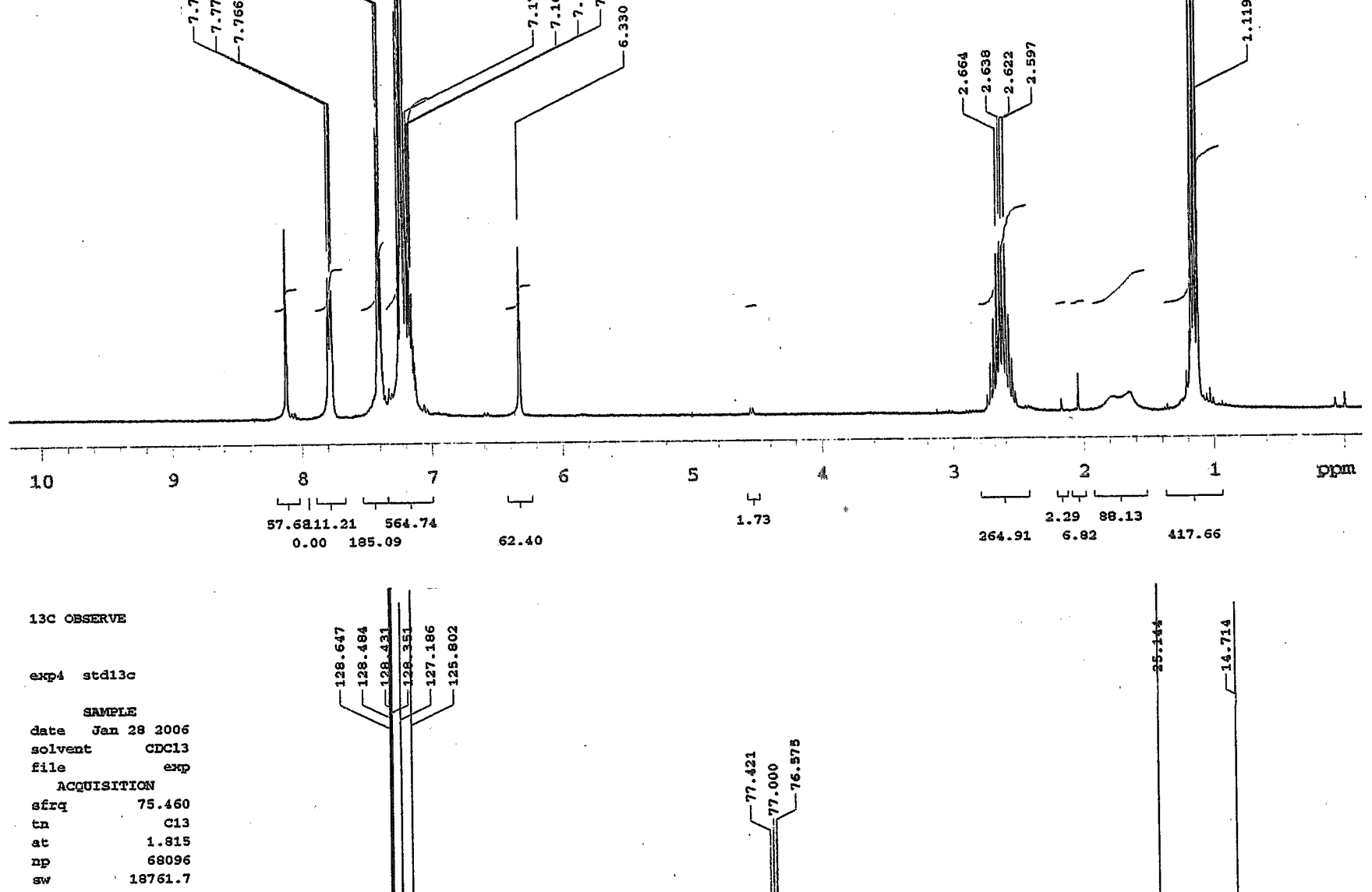

2.40

$\begin{array}{lll}64.91 & 6.82 & 417.66\end{array}$

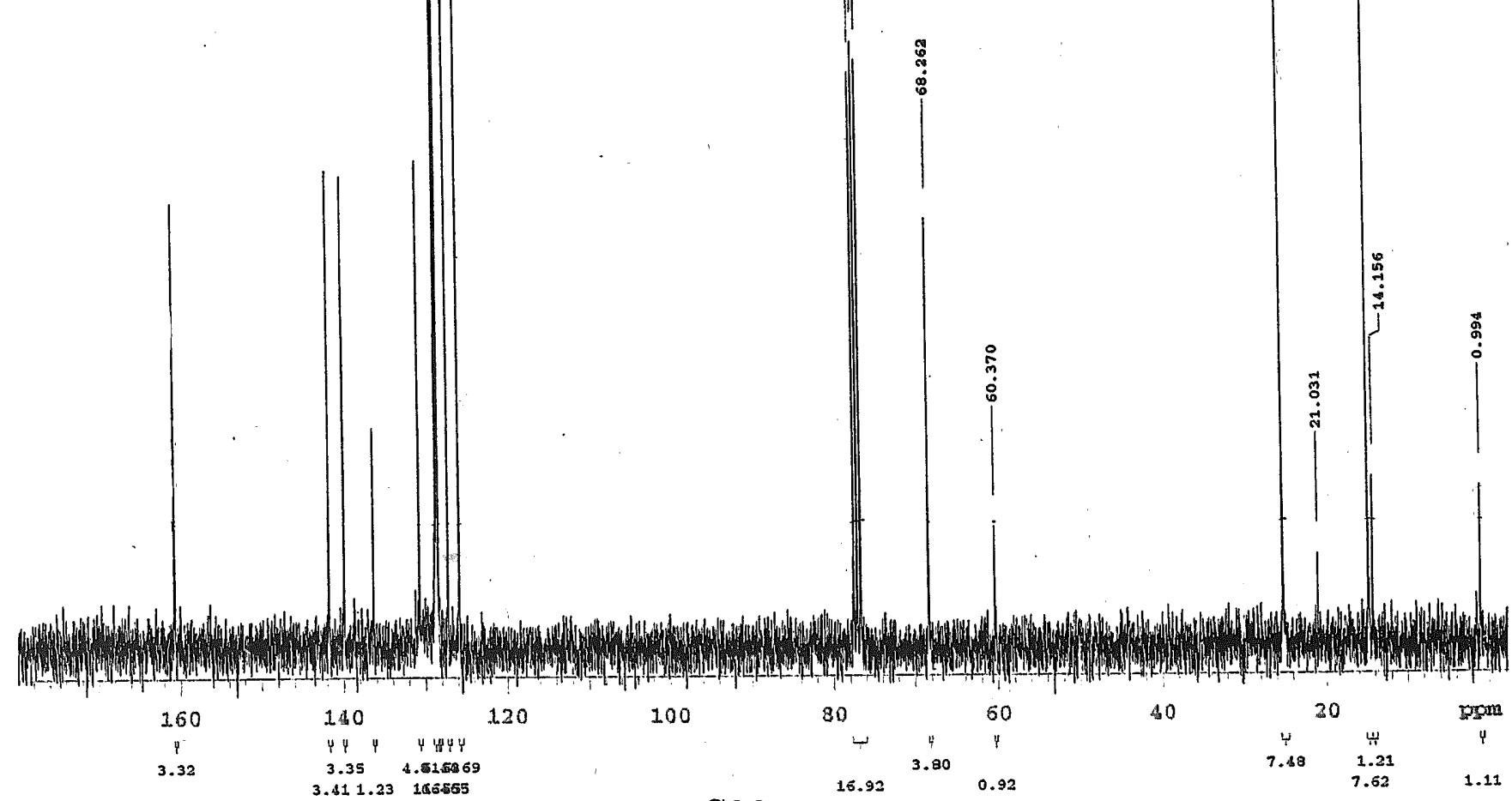



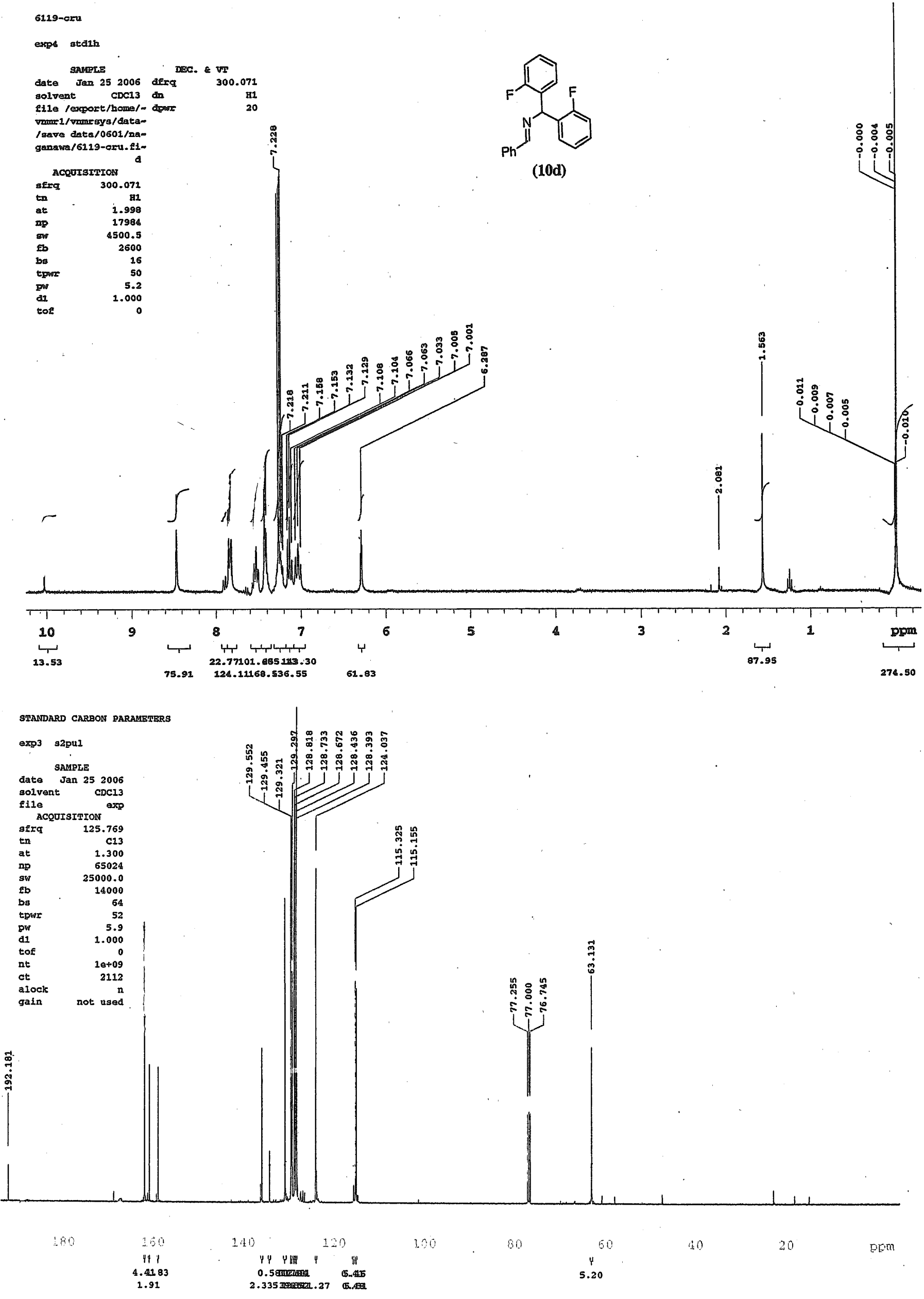
Fulse Sequence: s2pul

Solvent: CDC13

Arabient tercepature

GDASINI-300BB "varian"

Relaxk. delay 1.000 sec

Fulse 15.0 degrees

Acq. time $1.998 \mathrm{sec}$

Width $4500.5 \mathrm{kz}$

16 repet

OBSRRVE H1, $300.0695926 \mathrm{MHZ}$

DATA PROCESSINO

Tize 32768<smiles>Fc1ccc(/C=N/C(c2ccccc2F)c2ccccc2F)cc1</smiles>

(10e)

Total time 0 min, 49 soe

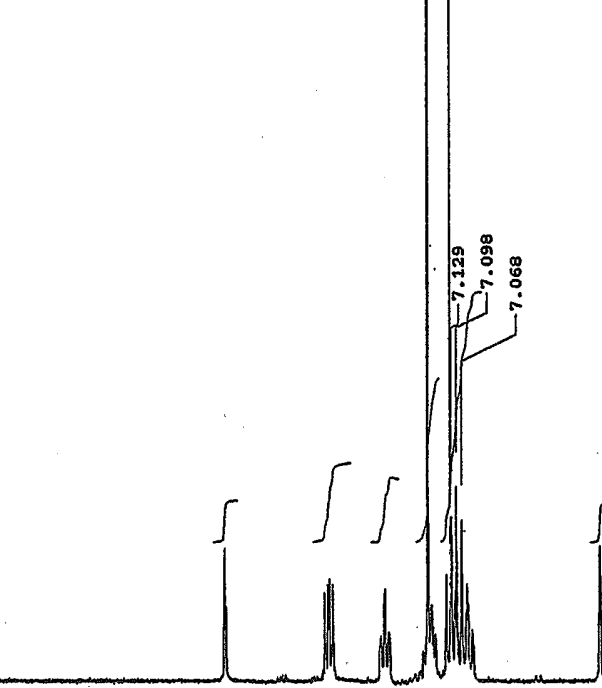
2 7

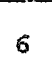

5 $106.22 \$ 18.49$
133.85276 .09

69.39

STAMDARD CARBON PARAMENIERS

Pulse Sequence: s2pul Solvent: $\operatorname{cDCl} 3$ Ambient temperature User: 1-14-87? INOVA-500. "varian"

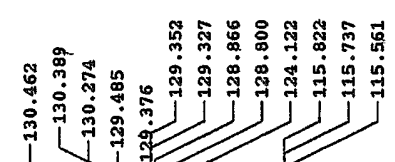

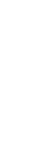
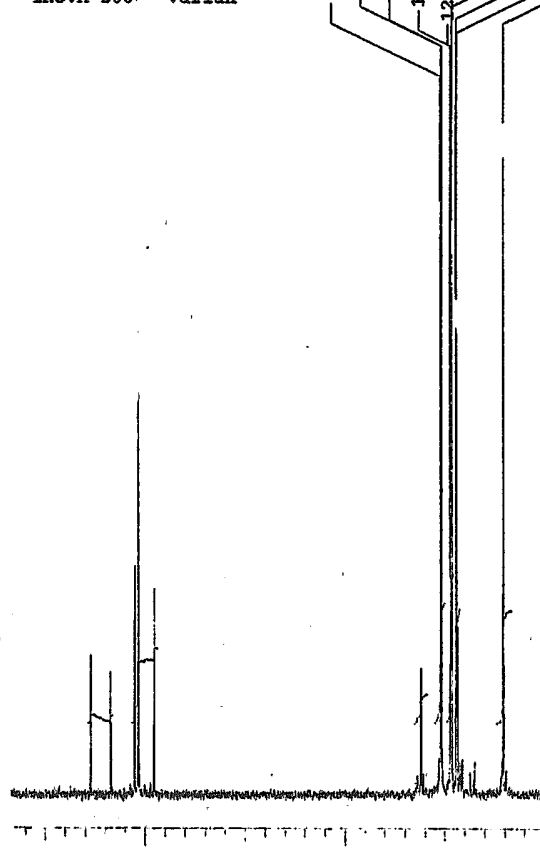

160

140

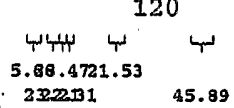

100

لهبا لها'

13.99

5.88 .4721
232201

45.89
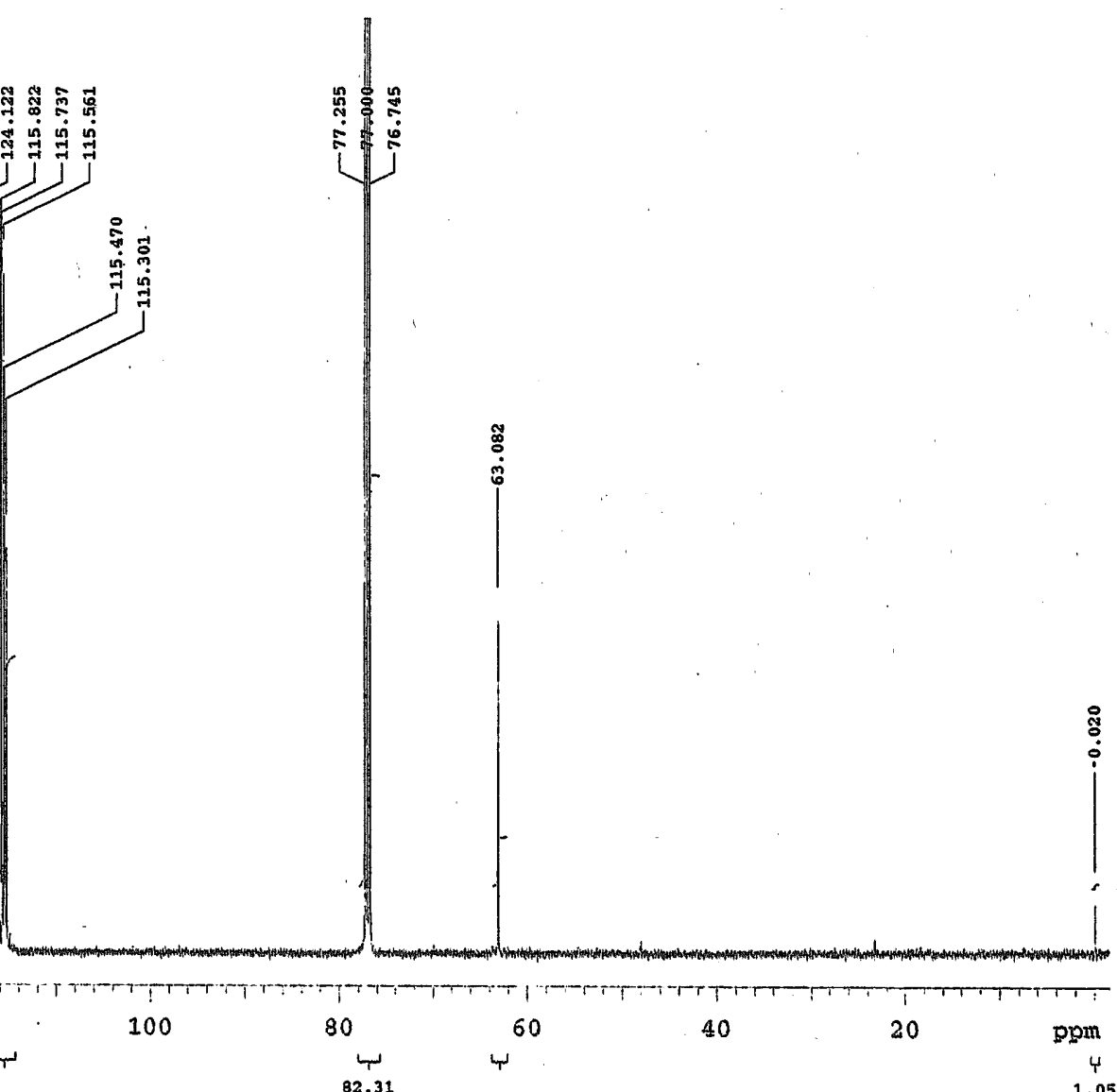

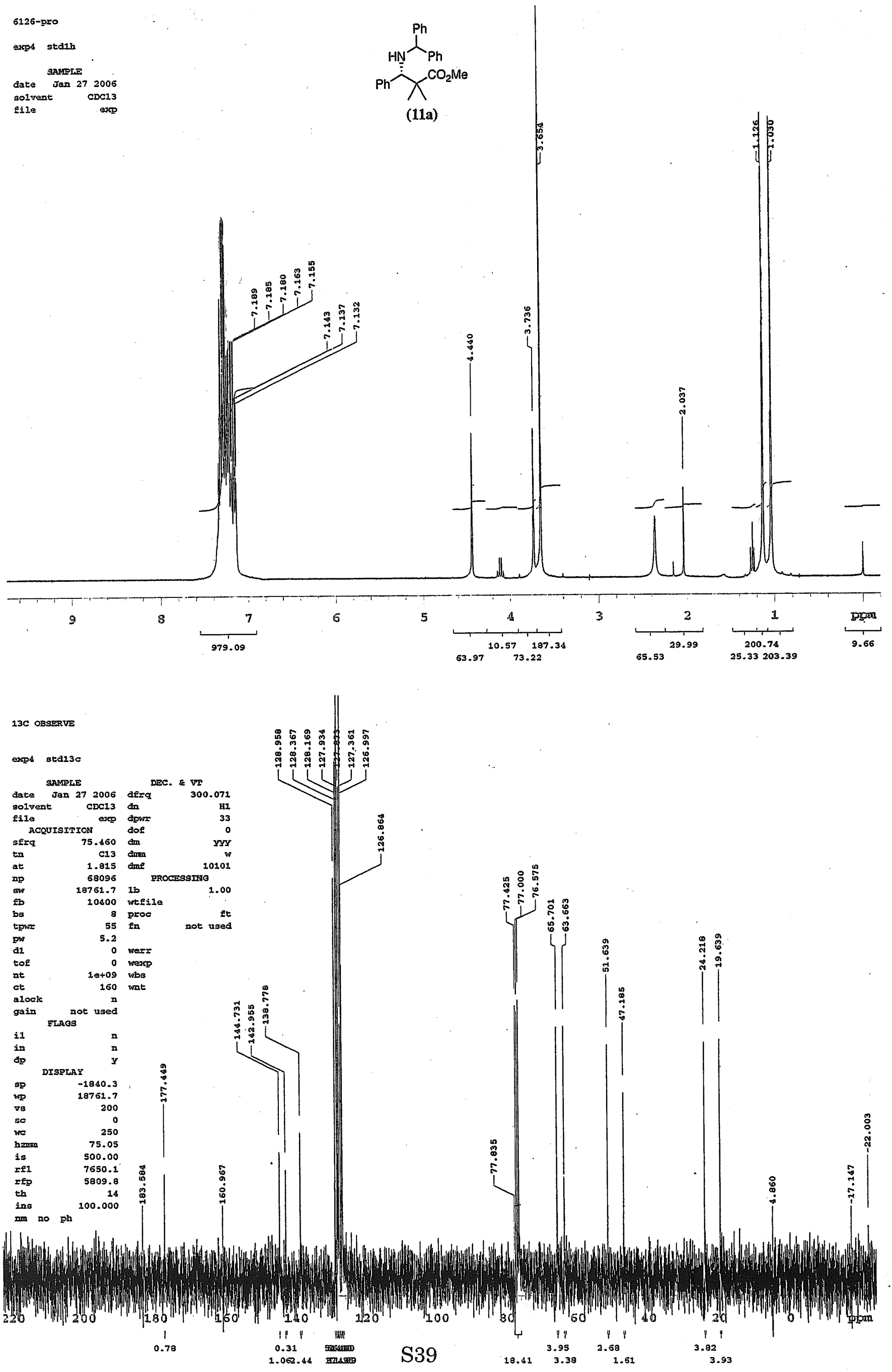

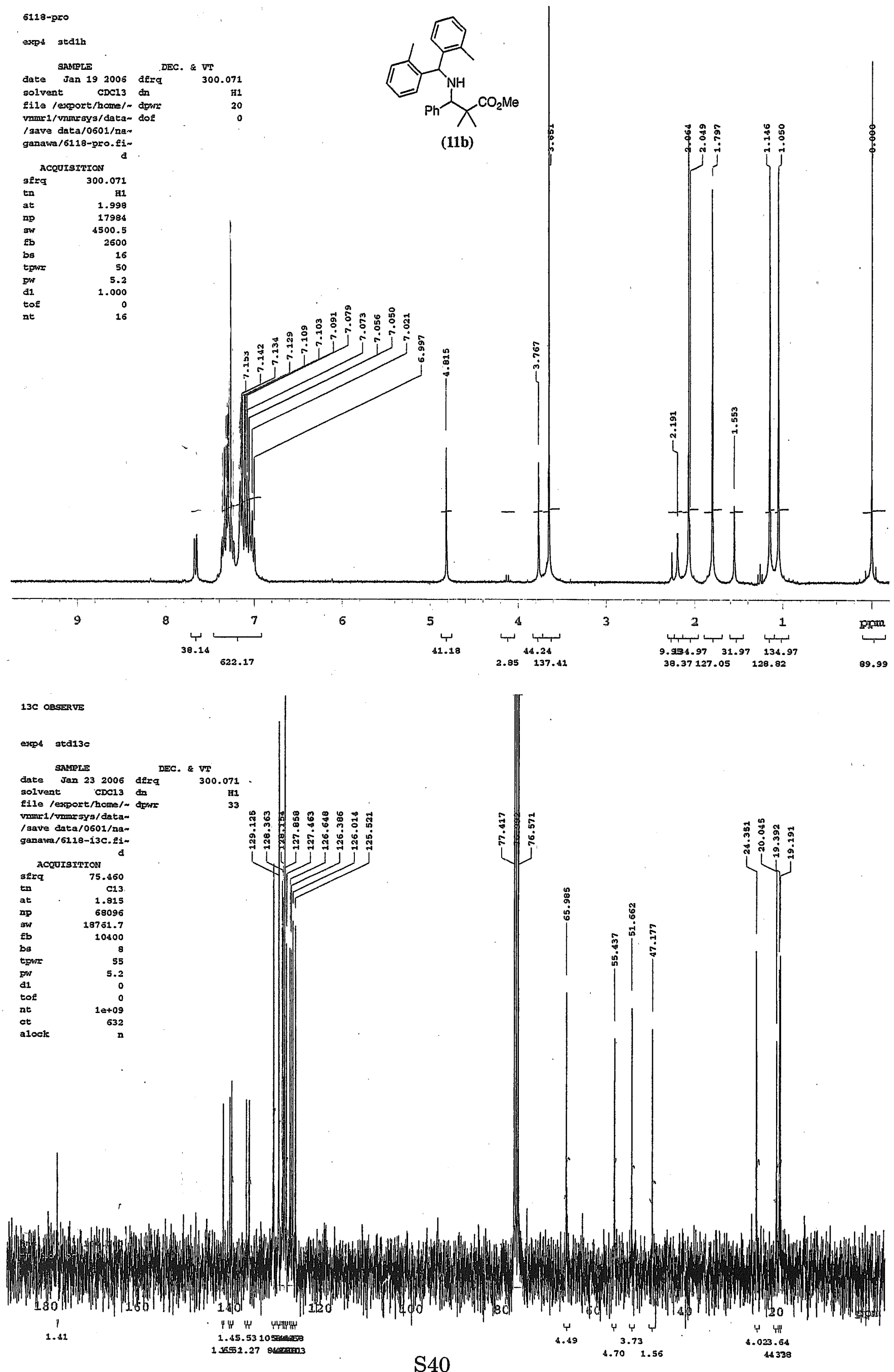

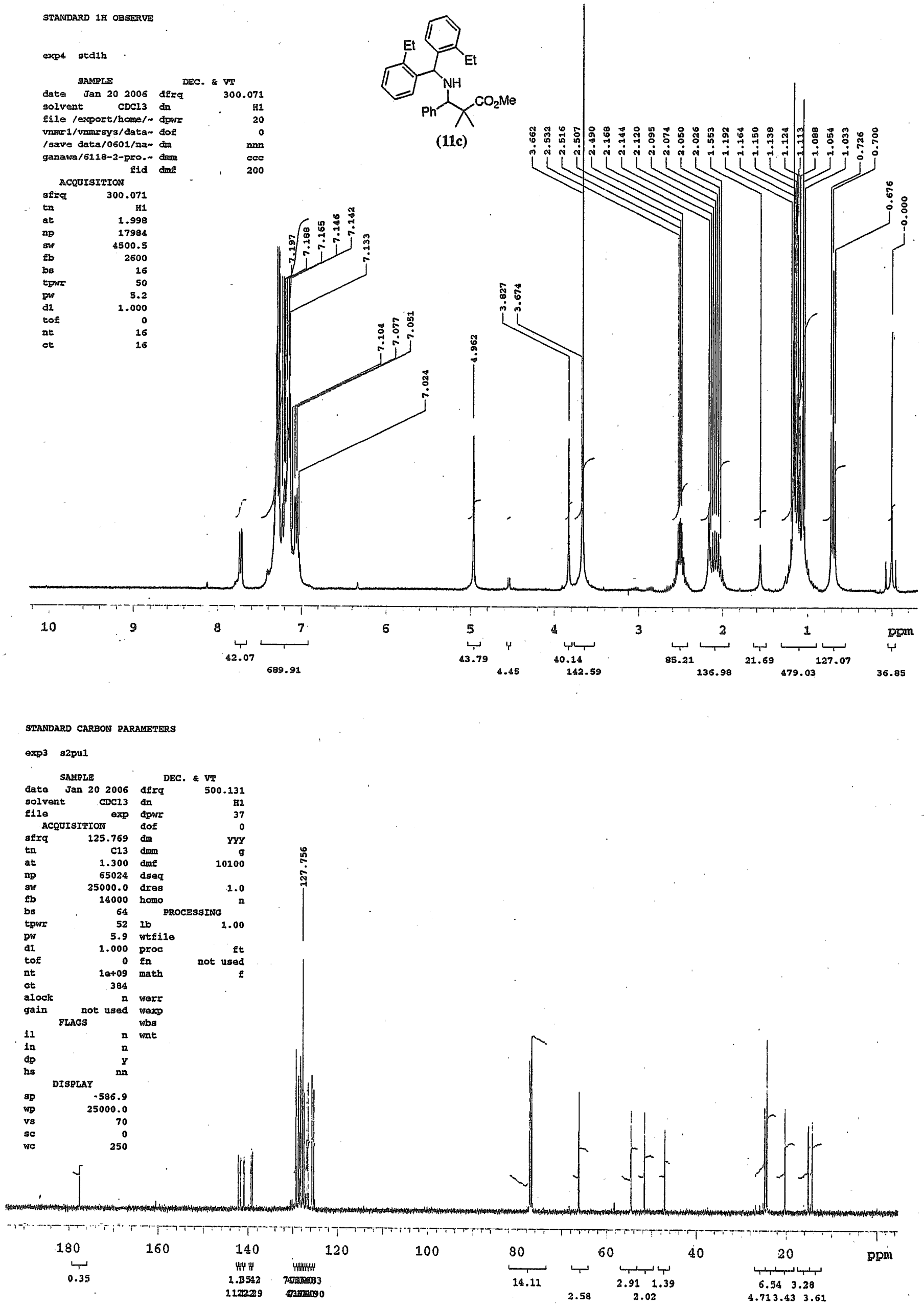

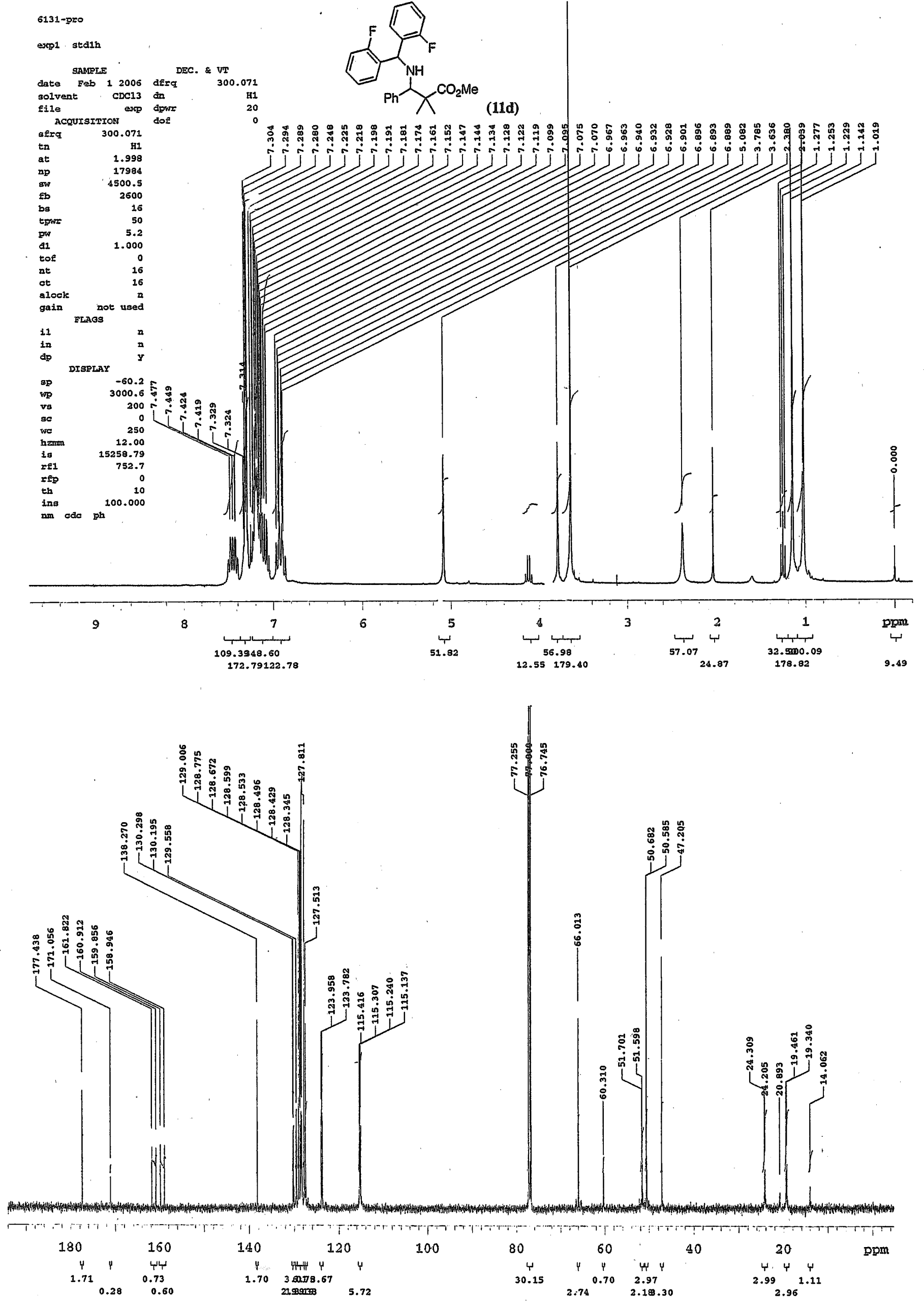

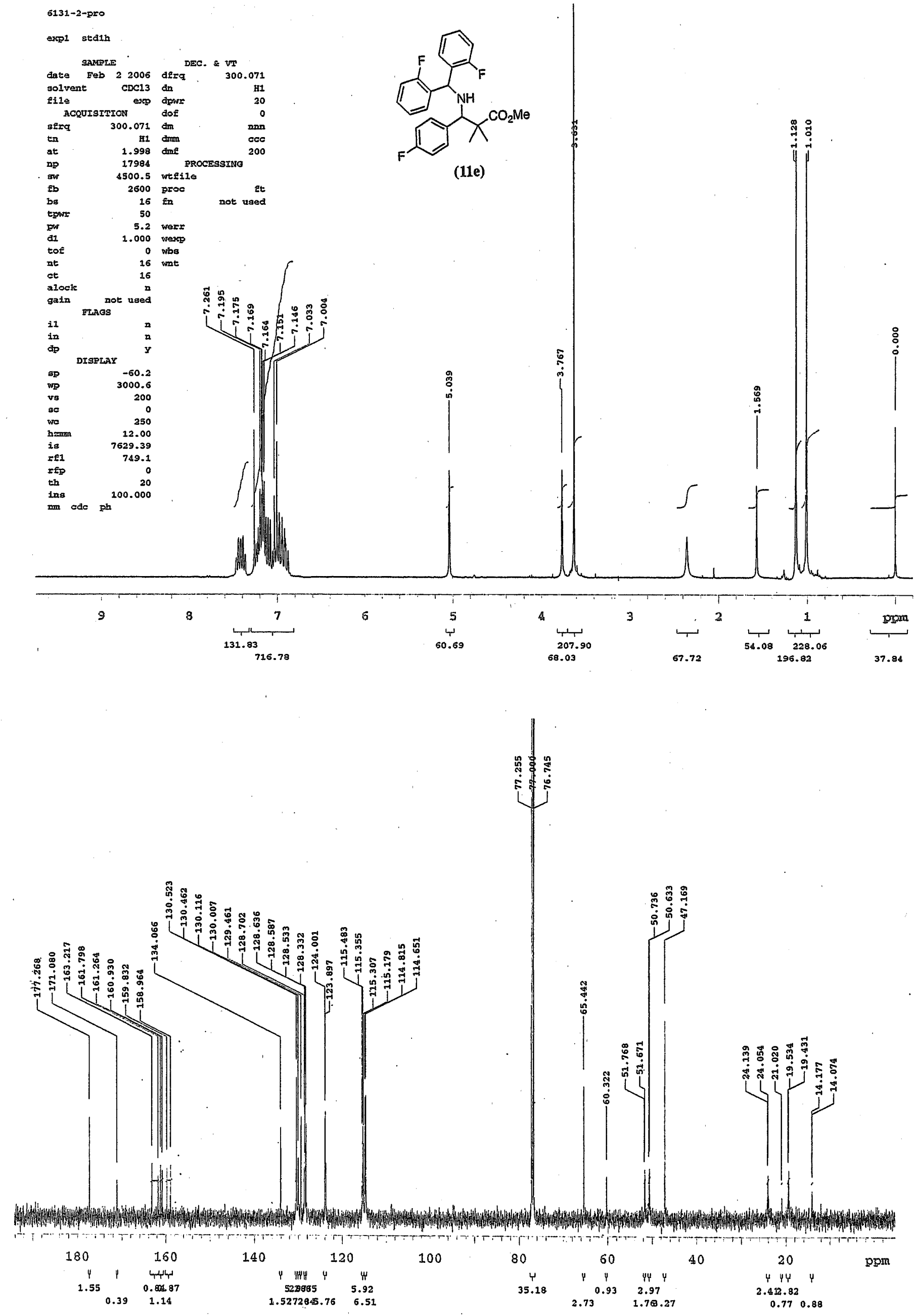